\title{
Geometry of Multiprimary Display Colors II: Metameric Control Sets and Gamut Tiling Color Control Functions
}

\author{
Carlos Eduardo Rodríguez-Pardo and Gaurav Sharma
}

\begin{abstract}
For multiprimary displays that have four or more primaries, a color may be reproduced using multiple alternative control vectors. We provide a complete characterization of the Metameric Control Set (MCS), i.e., the set of control vectors that reproduce a given color on the display. Specifically, we show that MCS is a convex polytope whose vertices are control vectors obtained from (parallelepiped) tilings of the gamut, i.e., the range of colors that the display can produce. The mathematical framework that we develop: (a) characterizes gamut tilings in terms of fundamental building blocks called facet spans, (b) establishes that the vertices of the MCS are fully characterized by the tilings of the gamut, and (c) introduces a methodology for the efficient enumeration of gamut tilings. The framework reveals the fundamental inter-relations between the geometry of the MCS and the geometry of the gamut developed in a companion Part I paper, and provides insight into alternative strategies for color control. Our characterization of tilings and the strategy for their enumeration also advance knowledge in geometry, providing new approaches and computational results for the enumeration of tilings for a broad class of zonotopes in $\mathbb{R}^{3}$.
\end{abstract}

Index Terms-multiprimary displays, metameric control sets, color gamut, color control, color control function, zonotope tiling, polar zonohedra

\section{INTRODUCTION}

Additive displays systems reproduce color by combining light emitted by a set of sources known as primaries. A control vector, which represents the relative intensity of the primaries, determines the color reproduced by the display. The set of all colors reproducible on the display through the use of alternative control vectors constitutes the gamut of the display. Displays with four or more primaries are known as multiprimary displays, and a key attribute of multiprimary displays is their flexibility for color control, as colors in the gamut may be produced by multiple different primary combinations. Characterizations of the gamut and the color control flexibility are important and useful because of their fundamental roles in the color management and in the design of multiprimary displays. In a companion Part I paper [1], we provided a complete geometrical representation for the gamut of multiprimary displays; including a tiling of the gamut with parallelepipeds generated by 3-tuples of primaries. In this Part II paper, we introduce and characterize the set of control vectors that reproduce a color in the gamut; a

G. Sharma is with the Department of Electrical and Computer Engineering, University of Rochester, Rochester, NY, 14627-0126 USA e-mail:

C.E. Rodríguez-Pardo is with the Department of Electrical and Computer Engineering, University of Rochester, Rochester, NY, 14627-0126 USA email: set that we refer to as the Metameric Control Set (MCS). Our characterization relates the geometry of the MCS with the gamut tilings obtained in the companion Part I paper and highlights the fact that the methodology introduced there provides only an incomplete set of tilings, therefore, only an incomplete characterization of the MCS. In this paper, we completely characterize the tilings of the gamut and establish that these characterize the MCS for each color in the gamut.

This manuscript is organized as follows. After Section II introduces notation and the context for the paper, Section III establishes the MCS as a convex polytope that can be characterized by its vertices and formulates an orthogonal decomposition of the MCS that can be used for the visualization of the MCS. Section IV introduces the concept of a facet span, which is then used to characterize the full set of tilings of the gamut and to establish, for each tristimulus, the equivalence between the vertices of the MCS and the control vectors associated with the tilings. Finally, Section V develops an efficient methodology and algorithm for enumerating all the gamut tilings. The paper concludes with a discussion in Section VI and a summary of key results in Section VII. Appendices A-E include proofs of the mathematical results, specifications of the multiprimary systems used as examples in the paper, and supplementary detail for the algorithm presented in Section V. Supplementary Materials included with this paper provide additional visualizations of MCSs, facet spans, complete sets, and tilings and summarize the computational time requirements for the tiling enumeration results presented in this paper.

\section{BACKGROUND AND PROBLEM FRAMEWORK}

We refer the reader to Section II of the companion Part I paper [1] for a summary of the common notational conventions and background that are also used in this paper. We introduce the few additional conventions and definitions that we require in this section and also quickly describe the problem framework and recapitulate the most relevant concepts and results from the companion Part I paper. Table I lists the notational conventions and acronyms. For a non-empty sequence of integer indices $\mathbb{I}$, we denote the mirror sequence by $\tilde{\mathbb{I}}$, where $\tilde{\mathbb{I}}[l]=\mathbb{I}[\mathscr{N}(\mathbb{I})-l+1]$, for all $l \in\langle\mathscr{N}(\mathbb{I})\rangle$, where $\mathbb{I}[l]$ denotes the $l^{\text {th }}$ index in $\mathbb{I}$ and $\mathscr{N}(\mathbb{I})$ denotes the cardinality of the set $\mathbb{I}$. A polytope in $\mathbb{R}^{N}$ obtained as an affine map of $[0,1]^{M}, M \geq 0$, into $\mathbb{R}^{N}$ is called a zonotope [2, Chap. 7]. For a $3 \times M$ matrix $\mathbf{A}=\left[\mathbf{a}_{1}, \mathbf{a}_{2}, \ldots, \mathbf{a}_{M}\right]$, and a $3 \times 1$ 
vector $\mathbf{v}$, we denote by $\mathcal{S}(\mathbf{v}, \mathbf{A}) \equiv\left\{\mathbf{A} \boldsymbol{\alpha}+\mathbf{v} \mid \boldsymbol{\alpha} \in[0,1]^{M}\right\}$ the region of tristimulus space spanned by $\mathbf{A}$ and located at the offset $\mathbf{v}$, also referred to as the origin of $\mathcal{S}(\mathbf{v}, \mathbf{A})$. For a $3 \times 3$ matrix $\mathbf{A}$ and a $3 \times 2$ matrix $\mathbf{B}$, both with linearly independent column vectors, and $3 \times 1$ vectors $\mathbf{v}$ and $\mathbf{c} \neq \mathbf{0}$, the sets $\mathcal{P}(\mathbf{v}, \mathbf{A}) \equiv \mathcal{S}(\mathbf{v}, \mathbf{A}), \mathcal{F}(\mathbf{v}, \mathbf{B}) \equiv \mathcal{S}(\mathbf{v}, \mathbf{B})$, and $\mathcal{E}(\mathbf{v}, \mathbf{c}) \equiv \mathcal{S}(\mathbf{v}, \mathbf{c})$ denote, respectively, a parallelepiped, a parallelogram, and a line segment, each having origin $\mathbf{v}$; each of these is a zonotope in $\mathbb{R}^{3}$.

TABLE I

LIST OF SYMBOLS/ACRONYMS

\begin{tabular}{|c|c|}
\hline Symbol/Acronym & Description \\
\hline \multicolumn{2}{|c|}{ Scalars, Sets, Indices } \\
\hline$K$ & Number of primaries \\
\hline$n, m, N, M$ & Generic integers \\
\hline$\langle M\rangle$ & The set of integers $\{1,2,3, \ldots, M\}$ \\
\hline $\mathcal{A}$ & Generic set \\
\hline $\mathscr{N}(\mathcal{A})$ & Cardinality of $\mathcal{A}$ \\
\hline $\mathbb{I}$ & Generic sequence of indices \\
\hline$\tilde{\mathbb{I}}$ & Mirror sequence of $\mathbb{I}$ \\
\hline $\mathbb{J}, \mathbb{P}$ & Sequences of two, three indices \\
\hline $\mathfrak{C}^{m}(\mathbb{I})$ & Set of all combinations of $m$ elements from $\mathbb{I}$ \\
\hline $\mathfrak{P}(\mathbb{I})$ & Set of all permutations of the elements in $\mathbb{I}$ \\
\hline \multicolumn{2}{|c|}{ Tristimulus and Gamut } \\
\hline $\mathbf{t}$ & Generic tristimulus \\
\hline$t_{0}, t_{1}$ & Display black and display white \\
\hline p & Primary tristimulus \\
\hline $\mathbf{P}$ & $3 \times K$ primary matrix \\
\hline $\mathcal{G}$ & Display gamut \\
\hline \multicolumn{2}{|c|}{ Control Vectors and Metameric Control Sets } \\
\hline MCS & Metameric Control Set \\
\hline CBS & Control Black Subspace \\
\hline CVS & Control Visual Subspace \\
\hline$\alpha, \omega$ & Generic $K \times 1$ control vector \\
\hline$\Omega(\mathbf{t})$ & MCS for $\mathbf{t}$ \\
\hline $\mathcal{V}_{\Omega(\mathbf{t})}$ & Set of vertices of the MCS $\Omega(\mathbf{t})$ \\
\hline $\begin{array}{l}\boldsymbol{\alpha}_{\Upsilon}, \boldsymbol{t}\left(\boldsymbol{\alpha}_{\Phi}\right. \\
\boldsymbol{\beta}, \Xi(\mathbf{t})\end{array}$ & $\begin{array}{l}\text { CVS and CBS components of } \boldsymbol{\alpha} \\
(K-3) \text {-dimensional CBS coordinate representation of } \boldsymbol{\alpha} \text { and } \\
\Omega(\mathbf{t}) \text { in an orthonormal basis for CBS }\end{array}$ \\
\hline \multicolumn{2}{|l|}{ Facet spans } \\
\hline $\mathcal{Z}^{(\mathbb{J}, \mathbb{I})}$ & Facet span, $\mathbb{J} \in \mathfrak{C}^{m}(\langle K\rangle), \mathbb{I} \in \mathfrak{P}(\langle K\rangle \backslash \mathbb{J})$ \\
\hline $\mathcal{P}_{l}^{(\mathbb{J}, \mathbb{I})}$ & 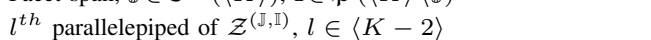 \\
\hline $\mathcal{F}_{l-1}^{(\mathbb{J}, \mathbb{I})}, \mathcal{F}_{l}^{(\mathbb{J}, \mathbb{I})}$ & The pair of facets of $\mathcal{P}_{l}^{(\mathbb{J}, \mathbb{I})}$ spanned by $\mathbf{P}[\mathbb{J}]$ \\
\hline $\mathbf{d}_{l}^{\mathbb{J}+}(\mathbb{I})$ & Origin of $\mathcal{F}_{l}^{(\mathbb{J}, \mathbb{I})}$ \\
\hline K & Generic set of compatible facet spans \\
\hline \multicolumn{2}{|c|}{ Collections of Parallelepipeds } \\
\hline $\mathcal{T}$ & Generic collection of parallelepipeds \\
\hline $\mathcal{T}_{\mathcal{K}}$ & Collection of all parallelepipeds making up the facet spans of $\mathcal{K}$ \\
\hline \multicolumn{2}{|c|}{ Color Control Functions } \\
\hline $\mathrm{CCF}$ & Color Control Function \\
\hline $\mathscr{C}$ & CCF \\
\hline $\mathscr{C}_{T}$ & Tiling CCF associated with gamut tiling $\mathcal{T}$ \\
\hline
\end{tabular}

\section{A. Problem Framework}

A display system is characterized by its $(3 \times K)$ matrix of primaries $\mathbf{P}=\left[\mathbf{p}_{1}, \mathbf{p}_{2}, \ldots, \mathbf{p}_{K}\right]$, where $\mathbf{p}_{k}$ is the $3 \times 1$ vector of CIE XYZ tristimulus values for the $k^{\text {th }}$ primary. A color is produced by an additive combination of the primaries that is described by a color control vector $\boldsymbol{\alpha}=\left[\alpha_{1}, \alpha_{2}, \ldots, \alpha_{K}\right]^{T}$, where $\alpha_{k} \in[0,1]$ is the control value that indicates the relative intensity of primary $\mathbf{p}_{k}$. The reproduced color, as a function of $\boldsymbol{\alpha}$, is represented by the $3 \times 1$ tristimulus
$\mathbf{t}(\boldsymbol{\alpha})=\mathbf{P} \boldsymbol{\alpha}+\mathbf{t}_{\mathbf{0}}=\left[t_{X}, t_{Y}, t_{Z}\right]^{T}$, where $\mathbf{t}_{\mathbf{0}} \stackrel{\text { def }}{=} \mathbf{t}(\mathbf{0})$ is the display black and $\mathbf{t}_{\mathbf{1}} \stackrel{\text { def }}{=} \mathbf{t}(\mathbf{1})$ is the display white, which are the tristimuli obtained when all primaries are turned completely off and on, respectively. Throughout the paper, we assume that any three columns of $\mathbf{P}$ are linearly independent. Noting that $[0,1]^{K}$ defines the domain of all possible primary combinations, the (tristimulus) gamut of the display $\mathcal{G} \stackrel{\text { def }}{=} \mathbf{t}\left([0,1]^{K}\right)=\left\{\mathbf{t}(\boldsymbol{\nu}) \mid \boldsymbol{\nu} \in[0,1]^{K}\right\}$ defines the range of (tristimulus) colors that the display can reproduce. Because $\mathcal{G}$ is an affine map of $\mathbb{R}^{K}$ into $\mathbb{R}^{3}, \mathcal{G}$ is a zonotope whose geometry we characterized in the companion Part I paper [1]. A color control function $(C C F)$ is a function $\mathscr{C}: \mathcal{G} \rightarrow[0,1]^{K}$ that assigns to every $\mathbf{t} \in \mathcal{G}$ a control vector $\mathscr{C}(\mathbf{t})$. A tiling $\mathcal{T}$ of the gamut $\mathcal{G}$ is a collection of parallelepipeds spanned by sets of three primaries and covering the gamut, i.e., $\mathcal{G}=\bigcup_{\mathcal{P} \in \mathcal{T}} \mathcal{P}$, such that any two different parallelepipeds $\mathcal{P}, \mathcal{P}^{\prime} \in \mathcal{T}$ are spanned by different sets of primaries and their intersection $\mathcal{P} \cap \mathcal{P}^{\prime}$ is a face of both $\mathcal{P}$ and $\mathcal{P}^{\prime}$. The companion Part I paper offers a methodology for obtaining gamut tilings by building the collection of parallelepipeds in a progression dictated by the order of the primaries. A gamut tiling obtained from this methodology is referred to as a progressive tiling. The progressive tiling is composed of parallelepipeds $\mathcal{P}\left(\mathbf{c}^{\mathbb{P}}, \mathbf{P}[\mathbb{P}]\right)$, one for each $\mathbb{P} \in \mathfrak{C}^{3}(\langle K\rangle)$, where the origin $\mathbf{c}^{\mathbb{P}}=\mathbf{P} \boldsymbol{\alpha}^{\mathbb{P}}+\mathbf{t}_{\mathbf{0}}$ has a (binary) control vector $\boldsymbol{\alpha}^{\mathbb{P}} \in\{0,1\}^{K}$, whose entries corresponding to the indices in $\mathbb{P}$ are zero. The companion Part I paper also introduces the progressive tiling $C C F$, for which, the control vector for each $\mathbf{t} \in \mathcal{P}\left(\mathbf{c}^{\mathbb{P}}, \mathbf{P}[\mathbb{P}]\right), \mathbb{P} \in \mathfrak{C}^{3}(\langle K\rangle)$, is given by $\mathscr{C}(\mathbf{t})=\boldsymbol{\alpha}^{\mathbb{P}}+\mathbf{I}_{K}[\mathbb{P}] \mathbf{P}^{-1}[\mathbb{P}]\left(\mathbf{t}-\mathbf{c}^{\mathbb{P}}\right)$.

\section{Metameric Control Sets}

For $\mathbf{t} \in \mathcal{G}$, the metameric control set $(M C S) \Omega(\mathbf{t}) \stackrel{\text { def }}{=}$ $\left\{\boldsymbol{\nu} \in[0,1]^{K} \mid \mathbf{t}=\mathbf{P} \boldsymbol{\nu}+\mathbf{t}_{\mathbf{0}}\right\}$ is the set of all control vectors that reproduce the tristimulus $\mathbf{t}$. Note that for $K=3, \mathbf{P}$ is an nonsingular $3 \times 3$ primary matrix, so the MCS is the singleton $\Omega(\mathbf{t})=\left\{\mathbf{P}^{-1}\left(\mathbf{t}-\mathbf{t}_{\mathbf{0}}\right)\right\}$. For $K \geq 4$, the primary matrix $\mathbf{P}$ represents an under-determined linear system of equations, so $\Omega(\mathbf{t})$ may contain multiple control vectors. From its definition, the MCS $\Omega(\mathbf{t})$ is the intersection of the feasibility set $[0,1]^{K}$ delimiting the region in $\mathbb{R}^{K}$ corresponding to physically meaningful control vectors available to drive the display and the affine subspace $\Lambda(\mathbf{t})=\left\{\boldsymbol{\nu} \in \mathbb{R}^{K} \mid \mathbf{P} \boldsymbol{\nu}=\left(\mathbf{t}-\mathbf{t}_{\mathbf{0}}\right)\right\}$. Because $[0,1]^{K}$ and $\Lambda(\mathbf{t})$ are polyhedral sets and the former is bounded, the MCS $\Omega(\mathbf{t})=[0,1]^{K} \cap \Lambda(\mathbf{t})$ is a bounded polyhedral set. Because every bounded polyhedral set is a polytope [3, pp. 119-120], it follows that the MCS $\Omega(\mathbf{t})$ is a convex polytope with alternative representation as the convex hull of its vertices $\mathcal{V}_{\Omega(\mathbf{t})}$, i.e., $\Omega(\mathbf{t})=\operatorname{conv}\left\{\boldsymbol{\omega} \in \mathcal{V}_{\Omega(\mathbf{t})}\right\}$. The vertices of $\Omega(\mathbf{t})$ are characterized algebraically in the following theorem, whose proof is presented in Appendix A.

Theorem 1. For a tristimulus $\mathbf{t} \in \mathcal{G}$, a control vector $\boldsymbol{\omega} \in \Omega(\mathbf{t})$ is a vertex of the MCS, i.e. $\boldsymbol{\omega} \in \mathcal{V}_{\Omega(\mathbf{t})}$ if and only if (iff) $\boldsymbol{\omega}$ has at most three vector components in $(0,1)$.

Note that the previously introduced progressive tiling control vectors satisfy the property that only three vectors components of $\boldsymbol{\nu}$ can have values in $(0,1)$. As a direct corollary 
of Theorem 1, progressive-tiling control vectors are vertices of the MCS.

To facilitate representations of the MCS in a lower dimensional subspace that can be readily visualized, next, we develop a convenient subspace decomposition that was introduced in [4].

\section{A. Orthogonal Decomposition and Visualization of the MCS}

Observing that $\Lambda\left(\mathbf{t}_{\mathbf{0}}\right)$ is the null space of $\mathbf{P}$, we have the standard subspace decomposition [5, pp. 405],

$$
\mathbb{R}^{K}=\mathscr{R}\left(\mathbf{P}^{T}\right)+\Lambda\left(\mathbf{t}_{\mathbf{0}}\right),
$$

where $\mathscr{R}\left(\mathbf{P}^{T}\right)$ is the range of of the $K \times 3$ matrix $\mathbf{P}^{T}$. Because $\mathbf{P}$ is a matrix of rank $3, \mathscr{R}\left(\mathbf{P}^{T}\right)$ and $\Lambda\left(\mathbf{t}_{\mathbf{0}}\right)$ are subspaces of dimensionality 3 and $(K-3)$, respectively. We refer to $\mathscr{R}\left(\mathbf{P}^{T}\right)$ as the control visual subspace (CVS) and to $\Lambda\left(\mathbf{t}_{\mathbf{0}}\right)$ as the control black subspace (CBS) drawing upon the analogy with the human visual subspace and the metameric black subspace [6], [7].

Now consider a tristimulus $\mathbf{t} \in \mathcal{G}$. For a pair of control vectors $\boldsymbol{\alpha}_{1}, \boldsymbol{\alpha}_{2} \in \Omega(\mathbf{t})$, we have $\mathbf{P}\left(\boldsymbol{\alpha}_{1}-\boldsymbol{\alpha}_{1}\right)=\mathbf{0}$, whereby the difference $\left(\boldsymbol{\alpha}_{1}-\boldsymbol{\alpha}_{2}\right) \in \Lambda\left(\mathbf{t}_{\mathbf{0}}\right)$. Therefore, variations within the MCS $\Omega(\mathbf{t})$ are fully contained in the CBS and can be visualized for $K \leq 6$, as we show next. Let (the columns of) $\mathbf{B}$ be an orthonormal basis for $\Lambda\left(\mathbf{t}_{\mathbf{0}}\right)$. Then, from (1), we express $\boldsymbol{\alpha} \in \Omega(\mathbf{t})$ as

$$
\begin{aligned}
\boldsymbol{\alpha} & =\mathbf{P}^{T}\left(\mathbf{P P}^{T}\right)^{-1}\left(\mathbf{t}-\mathbf{t}_{\mathbf{0}}\right)+\mathbf{B} \boldsymbol{\beta}, \\
\stackrel{\text { def }}{=} & \boldsymbol{\alpha}_{\Upsilon}+\boldsymbol{\alpha}_{\Phi}
\end{aligned}
$$

where $\boldsymbol{\alpha}_{\Upsilon}=\mathbf{P}^{T}\left(\mathbf{P P}^{T}\right)^{-1}\left(\mathbf{t}-\mathbf{t}_{\mathbf{0}}\right)=\mathbf{P}^{T}\left(\mathbf{P P}^{T}\right)^{-1} \mathbf{P} \boldsymbol{\alpha}$ is the projection of $\boldsymbol{\alpha}$ onto the CVS, $\boldsymbol{\alpha}_{\Phi}=\mathbf{B} \boldsymbol{\beta}=\mathbf{B B}^{T} \boldsymbol{\alpha}$ is the projection of $\boldsymbol{\alpha}$ onto the CBS, and the $(K-3) \times 1$ vector $\boldsymbol{\beta}=$ $\mathbf{B}^{T} \boldsymbol{\alpha}$ is the coordinate representation of the CBS component of $\boldsymbol{\alpha}$ in the basis B. Fig. 1 schematically illustrates the aforementioned decomposition, where for convenient visualization, one-dimensional representations are used for CBS and CVS with a two-dimensional representation for the space of control vectors. Observing that the CVS component of $\boldsymbol{\alpha}$ is uniquely determined by $\mathbf{t}$, we obtain a similar decomposition for the $\operatorname{MCS} \Omega(\mathbf{t})$,

$$
\Omega(\mathbf{t})=\mathbf{P}^{T}\left(\mathbf{P P}^{T}\right)^{-1}\left(\mathbf{t}-\mathbf{t}_{\mathbf{0}}\right)+\mathbf{B} \Xi(\mathbf{t}),
$$

where $\Xi(\mathbf{t})=\left\{\mathbf{B}^{T} \boldsymbol{\nu} \mid \boldsymbol{\nu} \in \Omega(\mathbf{t})\right\}$ is the representation of the CBS component of $\Omega(\mathbf{t})$ in the basis B. Because $\Omega(\mathbf{t})$ is a convex polytope, $\Xi(\mathbf{t})$ is also a convex polytope with a set of vertices $\mathcal{V}_{\Xi(\mathbf{t})}=\left\{\mathbf{B}^{T} \boldsymbol{\omega} \mid \boldsymbol{\omega} \in \mathcal{V}_{\Omega(\mathbf{t})}\right\}$. We visualize $\boldsymbol{\alpha} \in \Omega(\mathbf{t})$ and $\Omega(\mathbf{t})$ using their $(K-3)$ dimensional CBS coordinate representations $\boldsymbol{\beta}=\mathbf{B}^{T} \boldsymbol{\alpha}$ and $\Xi(\mathbf{t})$, respectively.

Figures 2 (a) and (b) illustrate two examples of the (CBS coordinate representation of the) MCS polytope $\Xi(\mathbf{t})$ for $K=5$ and $K=6$ primary systems, respectively, as $(K-3)$ dimensional polytopes. The vertices of the MCS polytopes colored red in these figures correspond to the progressivetiling control vectors, whereas the clear/white vertices do not correspond to any progressive-tiling control vector. The examples illustrate that the progressive tiling control vectors

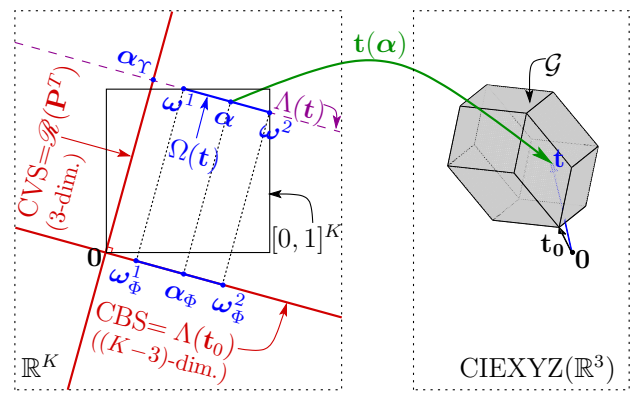

Fig. 1. Decomposition of MCS into CVS and CBS components. On the right, the CIEXYZ gamut $\mathcal{G}$ (solid-gray) for a $3 \times K$ primary matrix $\mathbf{P}$ with display black $\mathbf{t}_{\mathbf{0}}$ (black vector), and a tristimulus $\mathbf{t} \in \mathcal{G}$ (blue vector). On the left, the MCS $\Omega(\mathbf{t})$ represented by the solid-blue line that results from the intersection of the feasibility set $[0,1]^{K}$ (delimited by the solidblack square) and the affine subspace $\Lambda(t)$ (dashed-purple line). The control vectors $\boldsymbol{\alpha}, \boldsymbol{\omega}^{1}, \boldsymbol{\omega}^{2} \in \Omega(\mathbf{t})$ and the latter two are vertices of $\Omega(\mathbf{t})$ that are plotted as blue dots. The CVS and the CBS are represented by the orthogonal red lines, and the CVS and CBS components of $\boldsymbol{\alpha}, \boldsymbol{\omega}^{1}, \boldsymbol{\omega}^{2}$ and $\Omega(\mathbf{t})$ are plotted on the corresponding subspaces. The green line shows the mapping from the control vector $\boldsymbol{\alpha}$ to its corresponding tristimulus $\mathbf{t}(\boldsymbol{\alpha})$.

obtained via the methodology described in the companion Part I paper, do not define the full set of vertices of the MCS. This motivates the obvious question as to how the full set of vertices of the MCS can be characterized, which we answer next.

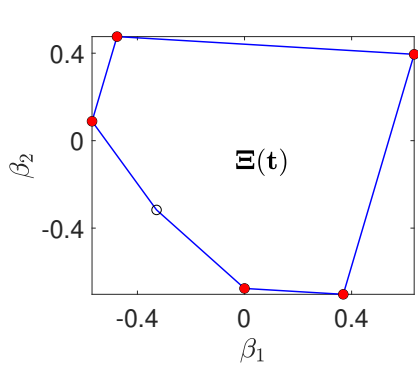

(a) $K=5$

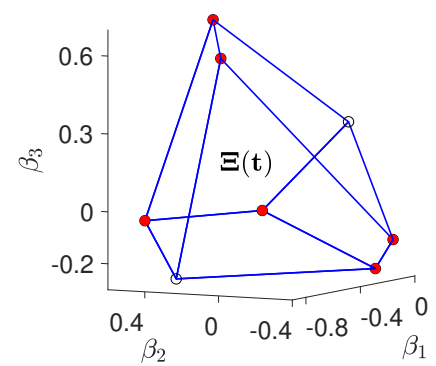

(b) $K=6$
Fig. 2. Example MCS polytopes $\Xi(\mathbf{t})$ in the $(K-3)$-dimensional CBS coordinate representations for the $K=5,6$ primary systems $\mathbf{P}_{w}^{(5)}, \mathbf{P}_{\mathscr{V}}^{(6)}$. See appendices $\mathrm{C}$ and $\mathrm{D}$ for the specifications of the primary matrices and the CBS bases used in this and subsequent figures.

\section{Facet Spans, Gamut Tilings, MCS Vertices}

We now introduce the concept of a facet span to build a framework for characterizing gamut tilings, which we subsequently use to characterize the vertices of MCS as tiling control vectors. Proofs of the lemmas and the theorem occurring in this section are presented in Appendix B.

\section{A. Facet Spans}

Recall from the companion Part I paper [1], that for each pair of indices $\mathbb{J} \in \mathfrak{C}^{2}(\langle K\rangle)$, the gamut boundary includes the pair of congruent parallelogram facets $\mathcal{F}\left(\mathbf{d}^{\mathbb{J}-}, \mathbf{P}[\mathbb{J}]\right)$ and $\mathcal{F}\left(\mathbf{d}^{\mathbb{J}+}, \mathbf{P}[\mathbb{J}]\right)$, spanned by the two primaries $\mathbf{P}[\mathbb{J}]$, where $\mathbf{d}^{\mathbb{I}-}=\mathbf{t}_{\mathbf{0}}+\mathbf{P} \boldsymbol{\chi}^{\mathbb{J}-}$ and $\mathbf{d}^{\mathbb{I}+}=\mathbf{t}_{\mathbf{0}}+\mathbf{P} \boldsymbol{\chi}^{\mathbb{J}+}$ are the facet origins, with control vectors $\chi^{\mathbb{J}-}=\left[\chi^{-}\left(\mathbf{u}_{\mathbb{J}}^{T} \mathbf{p}_{1}\right), \ldots, \chi^{-}\left(\mathbf{u}_{\mathbb{J}}^{T} \mathbf{p}_{K}\right)\right]^{T}$ and $\chi^{\mathbb{J}+}=\left[\chi^{+}\left(\mathbf{u}_{\mathbb{J}}^{T} \mathbf{p}_{1}\right), \ldots, \chi^{+}\left(\mathbf{u}_{\mathbb{J}}^{T} \mathbf{p}_{K}\right)\right]^{T}$, respectively, 
with $\mathbf{u}_{\mathbb{J}}$ denoting the normalized cross-product ${ }^{1} \mathbf{p}_{\mathbb{J}[1]} \otimes$ $\mathbf{p}_{\mathbb{J}[2]} /\left\|\mathbf{p}_{\mathbb{J}[1]} \otimes \mathbf{p}_{\mathbb{J}[2]}\right\|$. The complete gamut boundary $\mathcal{B}_{\mathcal{G}}$ is the essentially disjoint union of these $\left(\begin{array}{c}K \\ 2\end{array}\right)$ congruent pairs of facets. For a given $\mathbb{J} \in \mathfrak{C}^{2}(\langle K\rangle)$, via simple arithmetic manipulation, the facet origin $\mathbf{d}^{\mathbb{J}+}$ can be represented in terms of the facet origin $\mathbf{d}^{\mathbb{J}-}$ as

$$
\begin{aligned}
\mathbf{d}^{\mathbb{J}+} & =\mathbf{d}^{\mathbb{J}-}+\mathbf{P}\left(\chi^{\mathbb{J}+}-\chi^{\mathbb{J}-}\right) \\
& =\mathbf{d}^{\mathbb{J}-}+\sum_{j \in\langle K\rangle \backslash \mathbb{J}} \operatorname{sgn}\left(\mathbf{u}_{\mathbb{J}}^{T} \mathbf{p}_{j}\right) \mathbf{p}_{j} .
\end{aligned}
$$

Thus $\mathbf{d}^{\mathbb{J}+}$ is obtained from $\mathbf{d}^{\mathbb{J}-}$ through a series of signed displacements by all the other $(K-2)$ primaries, with the sign determined as indicated in (6). The commutativity of addition implies that the $(K-2)$ terms being added in the summation in (6) can be ordered in any sequence. We consider the sequence of points obtained by choosing a specific ordering. In particular, for any permutation $\mathbb{I} \in \mathfrak{P}(\langle K\rangle \backslash \mathbb{J})$ of the $(K-2)$ indices in $\langle K\rangle \backslash \mathbb{J}$, consider the sequence of tristimuli, $\mathbf{d}_{0}^{\mathbb{J}+}(\mathbb{I}), \mathbf{d}_{1}^{\mathbb{J}+}(\mathbb{I}), \ldots \mathbf{d}_{K-2}^{\mathbb{J}+}(\mathbb{I})$, obtained by using the corresponding order for the signed primary displacements in (6), viz., $\mathbf{d}_{0}^{\mathbb{I}+}(\mathbb{I})=\mathbf{d}^{\mathbb{I}-}$ and for $l=1,2, \ldots(K-2)$,

$$
\mathbf{d}_{l}^{\mathbb{J}+}(\mathbb{I})=\mathbf{d}^{\mathbb{J}-}+\sum_{j=1}^{l} \operatorname{sgn}\left(\mathbf{u}_{\mathbb{J}}^{T} \mathbf{p}_{\mathbb{I}[j]}\right) \mathbf{p}_{\mathbb{I}[j]} .
$$

Then, the same signed primary displacements, applied to the facet $\mathcal{F}\left(\mathbf{d}^{\mathbb{J}-}, \mathbf{P}[\mathbb{J}]\right)$ in the same sequence, delimit a region of the gamut that constitutes a fundamental building block of gamut tilings. More precisely, let $\mathcal{Z}^{(\mathbb{J}, \mathbb{I})}$ denote the sequence of $(K-2)$ parallelepipeds $\mathcal{P}_{1}^{(\mathbb{I}, \mathbb{I})}, \ldots, \mathcal{P}_{K-2}^{(\mathbb{J}, \mathbb{I})}$, where

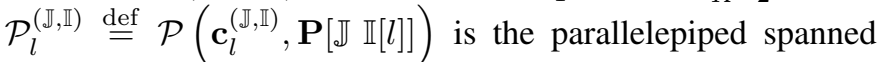
by $\mathbf{P}[\mathbb{I} \mathbb{I}[l]]$ with origin $\mathbf{c}_{l}^{(\mathbb{I}, \mathbb{I})}=\mathbf{d}_{l}^{\mathbb{I}+}(\mathbb{I})-\chi^{+}\left(\mathbf{u}_{\mathbb{J}}^{T} \mathbf{p}_{\mathbb{I}[l]}\right) \mathbf{p}_{\mathbb{I}[l]}$ (note that $\mathbf{c}_{l}^{(\mathbb{I}, \mathbb{I})} \in\left\{\mathbf{d}_{l}^{\mathbb{J}+}, \mathbf{d}_{l-1}^{\mathbb{J}+}\right\}$ ). We refer to $\mathcal{Z}^{(\mathbb{J}, \mathbb{I})}$ as the (gamut traversing) facet span corresponding to the gamut facets spanned by primaries indexed by $\mathbb{J}$ in the order $\mathbb{I}$ for the remaining $(K-2)$ primaries. We also use $\mathcal{Z}^{(\mathbb{I}, \mathbb{I})}$ to refer to the region formed by the union of the parallelepipeds, i.e.,

$$
\mathcal{Z}^{(\mathbb{I}, \mathbb{I})}=\bigcup_{l=1}^{(K-2)} \mathcal{P}_{l}^{(\mathbb{J}, \mathbb{I})} .
$$

The pair of facets of $\mathcal{P}_{l}^{(\mathbb{J}, \mathbb{I})}$ spanned by the two primaries indexed by $\mathbb{J}$ are denoted as $\mathcal{F}_{l-1}^{(\mathbb{J}, \mathbb{I})}$ and $\mathcal{F}_{l}^{(\mathbb{J}, \mathbb{I})}$, where $\mathcal{F}_{l}^{(\mathbb{I}, \mathbb{I})} \stackrel{\text { def }}{=} \mathcal{F}\left(\mathbf{d}_{l}^{\mathbb{J}+}(\mathbb{I}), \mathbf{P}[\mathbb{J}]\right)$ is the $l^{\text {th }}$ displacement of $\mathcal{F}\left(\mathbf{d}^{\mathbb{J}-}, \mathbf{P}[\mathbb{J}]\right)$, in the sequence defined by (7). Thus, $\mathcal{F}_{0}^{(\mathbb{J}, \mathbb{I})}, \ldots, \mathcal{F}_{K-2}^{(\mathbb{J}, \mathbb{I})}$ is a sequence of displaced congruent parallelograms. Observing that $\mathbf{d}_{l}^{\mathbb{I}+}(\mathbb{I})=\mathbf{d}_{l-1}^{\mathbb{I}+}(\mathbb{I})+$ $\operatorname{sgn}\left(\mathbf{u}_{\mathbb{J}}^{T} \mathbf{p}_{\mathbb{I}[l]}\right) \mathbf{p}_{\mathbb{I}[l]}$, and that $\mathbf{u}_{\mathbb{J}}^{T}\left(\mathbf{d}_{l}^{\mathbb{J}+}(\mathbb{I})-\mathbf{d}_{l-1}^{\mathbb{J}+}(\mathbb{I})\right)>0$, we see that $\mathbf{u}_{\mathbb{J}}^{T} \mathbf{d}_{0}^{\mathbb{I}+}(\mathbb{I}), \ldots, \mathbf{u}_{\mathbb{J}}^{T} \mathbf{d}_{K-2}^{\mathbb{J}+}(\mathbb{I})$ is a strictly increasing sequence, so the facet span $\mathcal{Z}^{(\mathbb{J}, \mathbb{I})}$ is the union of $(K-2)$ essentially disjoint parallelepipeds, with consecutive parallelepipeds intersecting in a common facet, $\mathcal{P}_{l-1}^{(\mathbb{J}, \mathbb{I})} \cap \mathcal{P}_{l}^{(\mathbb{J}, \mathbb{I})}=\mathcal{F}_{l-1}^{(\mathbb{J}, \mathbb{I})}$,

\footnotetext{
${ }^{1}$ To render our definition unambiguous, we assume throughout that a pair of indices $\mathbb{J}$ used in a cross-product definition are always ordered in increasing order.
}

for $l>1$. We note here that this "chaining property" also applies to progressive tilings obtained using the methodology developed in the companion Part I paper [1], as stated in Clause 9 of Theorem 1 in [1]. Fig. 3 illustrates a facet span for a $K=5$ primary system.

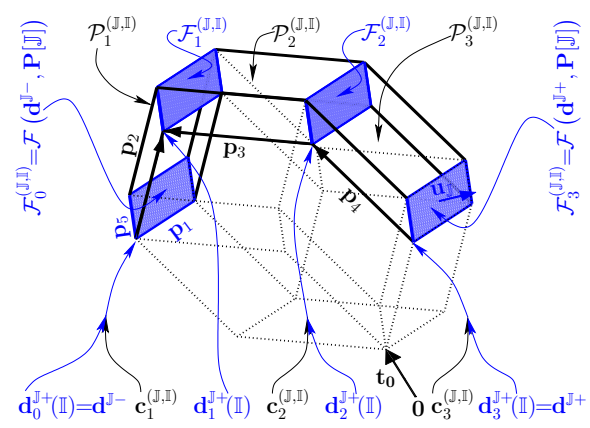

Fig. 3. Example facet span $\mathcal{Z}^{(\mathbb{J}, \mathbb{I})}$ for the $K=5$ primary display system $\mathbf{P}_{e}^{(5)}$, where $\mathbb{J}=[15] \in \mathfrak{C}^{2}(\langle K\rangle)$ and $\mathbb{I}=\left[\begin{array}{lll}2 & 3 & 4\end{array}\right] \in \mathfrak{P}(\langle K\rangle \backslash \mathbb{J})$.

Note that every facet span in a gamut with $K=3$ has only one parallelepiped, the gamut itself, thus, all facet spans are the same. For $K \geq 4$ and $\mathbb{J}, \mathbb{J}^{\prime} \in \mathfrak{C}^{2}(\langle K\rangle)$, note that $\mathbb{J} \neq \mathbb{J}^{\prime}$ implies that $\mathcal{Z}^{(\mathbb{J}, \mathbb{I})} \neq \mathcal{Z}^{\left(\mathbb{J}^{\prime}, \mathbb{I}^{\prime}\right)}$ for any $\mathbb{I}, \mathbb{I}^{\prime}$, while for $\mathbb{I}=\mathbb{J}^{\prime}$ different pair of sequences $\mathbb{I}, \mathbb{I}^{\prime}$ correspond to different pair of facet spans, as stated in following lemma.

Lemma 1 . Let $\mathbb{J} \in \mathfrak{C}^{2}(\langle K\rangle), \mathbb{I}, \mathbb{I}^{\prime} \in \mathfrak{P}(\langle K\rangle \backslash \mathbb{J})$, and $K \geq 4$. Then, $\mathcal{Z}^{(\mathbb{J}, \mathbb{I})}=\mathcal{Z}^{\left(\mathbb{J}, \mathbb{I}^{\prime}\right)}$, iff, $\mathbb{I}=\mathbb{I}^{\prime}$.

The number of distinct facet spans is immediately determined by Lemma 1, which we state as the following corollary.

Corollary 1. For each $\mathbb{J} \in \mathfrak{C}^{2}(\langle K\rangle)$, there are $(K-2)$ ! different facet spans spanned by the primaries identified by the indices $\mathbb{J}$. For $K \geq 4$, there are a total of $K ! / 2$ different facet spans.

Figure 4 illustrates all the facet spans for a $K=4$ primary configuration. Facet spans are building blocks of gamut tilings, as we show next.

\section{B. Gamut Tilings from Sets of Compatible Facet Spans}

We define compatibility for a pair of facet spans in terms of the pairwise relations between parallelepipeds from each facet span. Specifically, for $\mathbb{J}, \mathbb{J}^{\prime} \in \mathfrak{C}^{2}(\langle K\rangle), \mathbb{I} \in \mathfrak{P}(\langle K\rangle \backslash \mathbb{J})$, and $\mathbb{I}^{\prime} \in \mathfrak{P}\left(\langle K\rangle \backslash \mathbb{J}^{\prime}\right)$, we say that a pair of parallelepipeds $\mathcal{P}_{l}^{(\mathbb{J}, \mathbb{I})} \in \mathcal{Z}^{(\mathbb{J}, \mathbb{I})}$ and $\mathcal{P}_{l^{\prime}}^{\left(\mathbb{J}^{\prime}, \mathbb{I}^{\prime}\right)} \in \mathcal{Z}^{\left(\mathbb{J}^{\prime}, \mathbb{I}^{\prime}\right)}$, with $l, l^{\prime} \in\langle K-2\rangle$ are compatible if the intersection $\mathcal{P}_{l}^{(\mathbb{J}, \mathbb{I})} \cap \mathcal{P}_{l^{\prime}}^{\left(\mathbb{J}^{\prime}, \mathbb{I}^{\prime}\right)}$ is a face for both parallelepipeds, and furthermore, when $\mathbb{I} \cup \mathbb{I}[l]=$ $\mathbb{J}^{\prime} \cup \mathbb{I}^{\prime}\left[l^{\prime}\right]$, i.e., $\mathcal{P}_{l}^{(\mathbb{I}, \mathbb{I})}$ and $\mathcal{P}_{l^{\prime}}^{\left(\mathbb{J}^{\prime}, \mathbb{I}^{\prime}\right)}$ are spanned by the same primaries, we have $\mathcal{P}_{l}^{(\mathbb{J}, \mathbb{I})} \cap \mathcal{P}_{l^{\prime}}^{\left(\mathbb{J}^{\prime}, \mathbb{I}^{\prime}\right)}=\mathcal{P}_{l}^{(\mathbb{J}, \mathbb{I})}=\mathcal{P}_{l^{\prime}}^{\left(\mathbb{J}^{\prime}, \mathbb{I}^{\prime}\right)}$. For $\mathbb{J}, \mathbb{J}^{\prime} \in \mathfrak{C}^{2}(\langle K\rangle), \mathbb{I} \in \mathfrak{P}(\langle K\rangle \backslash \mathbb{J})$, and $\mathbb{I}^{\prime} \in \mathfrak{P}\left(\langle K\rangle \backslash \mathbb{J}^{\prime}\right)$, we say that the facet spans $\mathcal{Z}^{(\mathbb{J}, \mathbb{I})}$ and $\mathcal{Z}^{\left(\mathbb{J}^{\prime}, \mathbb{I}^{\prime}\right)}$ are compatible, if every pair of parallelepipeds $\mathcal{P}_{l}^{(\mathbb{I}, \mathbb{I})} \in \mathcal{Z}^{(\mathbb{J}, \mathbb{I})}$ and $\mathcal{P}_{l^{\prime}}^{\left(\mathbb{J}^{\prime}, \mathbb{I}^{\prime}\right)} \in$ $\mathcal{Z}^{\left(\mathbb{J}^{\prime}, \mathbb{I}^{\prime}\right)}$ is compatible.

We refer to the facet span $\mathcal{Z}^{(\mathbb{U}, \tilde{I})}$ as the mirror symmetric facet span for the facet $\operatorname{span} \mathcal{Z}^{(\mathbb{J}, \mathbb{I})}$. A pair of facet spans is compatible iff the corresponding pair of mirror symmetric 


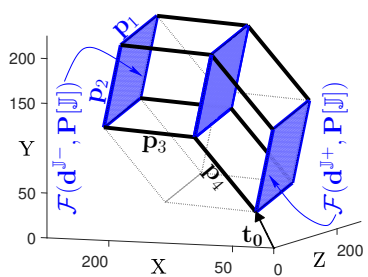

(a) $\mathbb{J}=[12], \mathcal{Z}^{(\mathbb{J},[34])}$

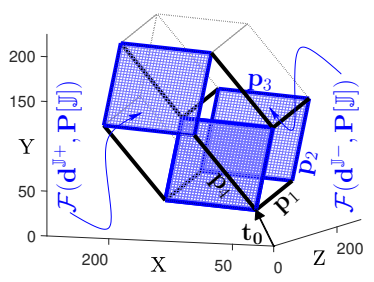

(e) $\mathbb{J}=[23], \mathcal{Z}^{(\mathbb{J},[14])}$

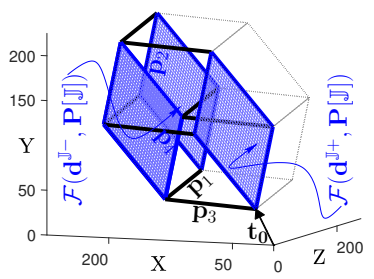

(i) $\mathbb{J}=[24], \mathcal{Z}^{\left(\mathbb{J},\left[\begin{array}{ll}1 & 3\end{array}\right]\right)}$

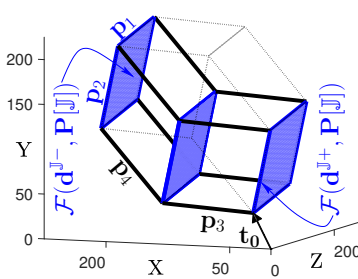

(b) $\mathbb{J}=[12], \mathcal{Z}^{(\mathbb{J},[43])}$

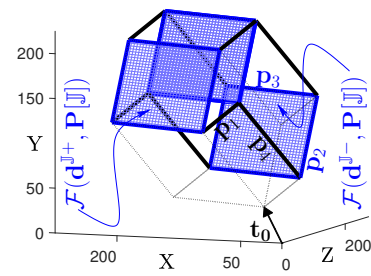

(f) $\mathbb{J}=[23], \mathcal{Z}^{\left(\mathbb{J},\left[\begin{array}{ll}4 & 1\end{array}\right]\right)}$

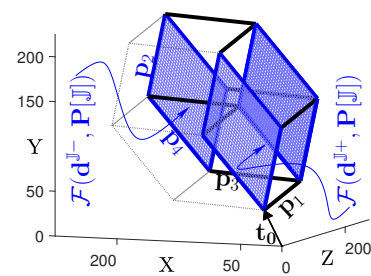

(j) $\left.\left.\mathbb{J}=[24], \mathcal{Z}^{(\mathbb{J},[3} 1\right]\right)$

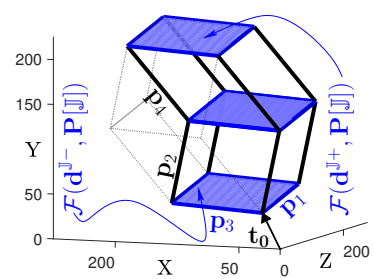

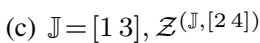

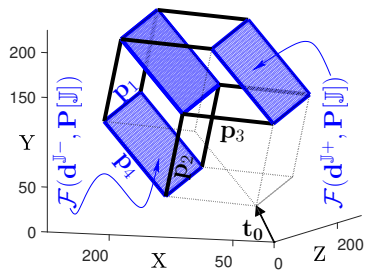

(g) $\mathbb{J}=[14], \mathcal{Z}^{(\mathbb{J},[23])}$

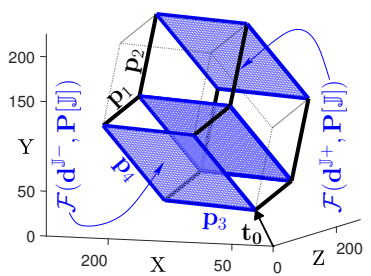

(k) $\mathbb{J}=[34], \mathcal{Z}^{\left(\mathbb{J},\left[\begin{array}{ll}1 & 2\end{array}\right]\right)}$

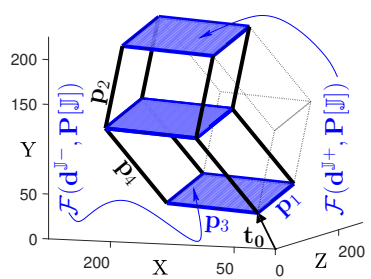

(d) $\mathbb{J}=[13], \mathcal{Z}^{(\mathbb{J},[42])}$

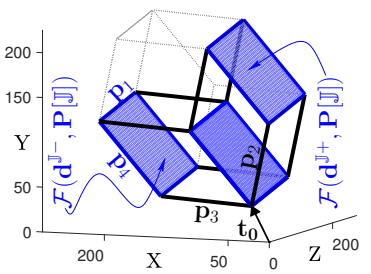

(h) $\mathbb{J}=\left[\begin{array}{ll}1 & 4\end{array}\right], \mathcal{Z}^{\left(\mathbb{J},\left[\begin{array}{ll}3 & 2\end{array}\right]\right)}$

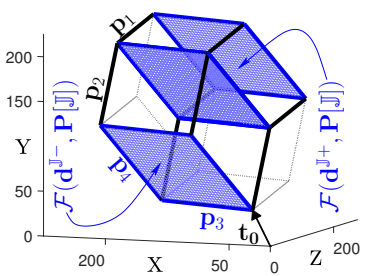

(l) $\mathbb{J}=[34], \mathcal{Z}^{\left(\mathbb{J},\left[\begin{array}{ll}2 & 1\end{array}\right]\right)}$

Fig. 4. All $K ! / 2=12$ facet spans for the $K=4$ primary display system $\mathbf{P}_{e}^{(4)}$ shown in the context of the display gamut (light-gray dashed-lines). Example pairs of compatible/incompatible facet spans include $\left.\left(\mathcal{Z}^{([12],[34])}, \mathcal{Z}^{\left([13],\left[{ }^{\ell}\right.\right.} 2\right]\right) /\left(\mathcal{Z}^{([14],[23])}, \mathcal{Z}^{([34],[12])}\right)$ and the corresponding mirror symmetric pairs

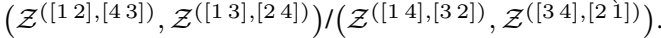

facet spans is compatible, a result that we state as the following lemma.

Lemma 2. Let $\mathbb{J}, \mathbb{J}^{\prime} \in \mathfrak{C}^{2}(\langle K\rangle), \mathbb{I} \in \mathfrak{P}(\langle K\rangle \backslash \mathbb{J})$, and $\mathbb{I}^{\prime} \in \mathfrak{P}\left(\langle K\rangle \backslash \mathbb{J}^{\prime}\right)$. Then, $\mathcal{Z}^{(\mathbb{J}, \mathbb{I})}$ and $\mathcal{Z}^{\left(\mathbb{J}^{\prime}, \mathbb{I}^{\prime}\right)}$ are compatible iff $\mathcal{Z}^{(\mathbb{I}, \tilde{\mathbb{I}})}$ and $\mathcal{Z}^{\left(\mathbb{J}^{\prime}, \tilde{\mathbb{I}}^{\prime}\right)}$ are compatible.

Now, let $\mathcal{K}$ be a set of pairwise compatible facet spans, and let $\mathcal{T}_{\mathcal{K}}=\left\{\mathcal{P}_{l}^{(\mathbb{J}, \mathbb{I})} \mid l \in\langle K-2\rangle, \mathcal{Z}^{(\mathbb{J}, \mathbb{I})} \in \mathcal{K}\right\}$ be the collection of all parallelepipeds making up the facet spans of $\mathcal{K}$. Observing that every pair of parallelepipeds in $\mathcal{T}_{\mathcal{K}}$ is compatible, we see that the number of distinct parallelepipeds in the collection is $\mathscr{N}\left(\mathcal{T}_{\mathcal{K}}\right) \leq\left(\begin{array}{c}K \\ 3\end{array}\right)$ and the distinct parallelepipeds are essentially disjoint. If $\mathscr{N}\left(\mathcal{T}_{\mathcal{K}}\right)=\left(\begin{array}{c}K \\ 3\end{array}\right)$, we say that the set $\mathcal{K}$ is maximal. Maximal sets produce gamut tilings as we establish in the following Lemma.

Lemma 3. The collection of parallelepipeds $\mathcal{T}_{\mathcal{K}}$ defined by a maximal set $\mathcal{K}$ is a gamut tiling.

Lemma 3 motivates the question on whether every gamut tiling can be obtained from a maximal set, which we answer next.

\section{Gamut Tilings as Maximal Sets}

A maximal set is called a complete set, if $\mathcal{K}$ includes a facet span for every primary pair, i.e., it has $\left(\begin{array}{c}K \\ 2\end{array}\right)$ facet spans, Figure 5 illustrates examples of maximal and complete sets for the $K=4$ primary system $\mathbf{P}_{e}^{(4)}$.

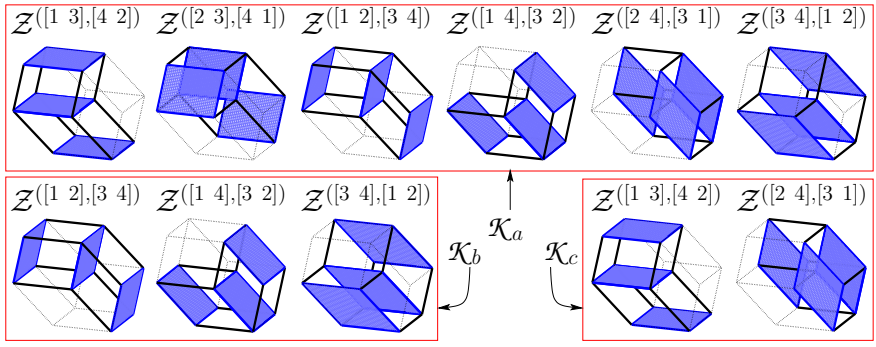

Fig. 5. Examples of complete and maximal sets of compatible facet spans for the $K=4$ primary display system $\mathbf{P}_{e}^{(4)}$. The sets of compatible facet spans $\mathcal{K}_{a}$ (top), $\mathcal{K}_{b}$ (bottom left) and $\mathcal{K}_{c}$ (bottom right) are all maximal sets because the $\mathscr{N}\left(\mathcal{T}_{\mathcal{K}_{a}}\right)=\mathscr{N}\left(\mathcal{T}_{\mathcal{K}_{b}}\right)=\mathscr{N}\left(\mathcal{T}_{\mathcal{K}_{c}}\right)=\left(\begin{array}{c}K \\ 3\end{array}\right)=4$; the associated collections of parallelepipeds are therefore gamut tilings. The set $\mathcal{K}_{a}$ is complete because it includes a facet span for each pair $(\mathbb{J})$ of primaries. For this example, $\mathcal{K}_{b}, \mathcal{K}_{c} \subset \mathcal{K}_{a}$, thus, $\mathcal{T}_{\mathcal{K}_{a}}=\mathcal{T}_{\mathcal{K}_{b}}=\mathcal{T}_{\mathcal{K}_{c}}$.

Lemma 4. Maximal sets satisfy the following properties:

1) The mapping $h: \mathcal{K} \mapsto \mathcal{T}_{\mathcal{K}}$ defines a bijection from complete sets to gamut tilings, i.e., there is a one-toone correspondence between complete sets and gamut tilings.

2) Every maximal set is a subset of one and only one complete set.

3) Every facet span belongs to some complete set.

4) A set $\mathcal{K}$ of facet spans is maximal iff the set $\tilde{\mathcal{K}}=$ $\left\{\mathcal{Z}^{(\mathbb{J}, \tilde{\mathbb{I}})} \mid \mathcal{Z}^{(\mathbb{J}, \mathbb{I})} \in \mathcal{K}\right\}$ is maximal.

Fig. 6 shows the collection of (all) complete sets for the for the $K=4$ primary system $\mathbf{P}_{e}^{(4)}$. Each of the two complete sets 


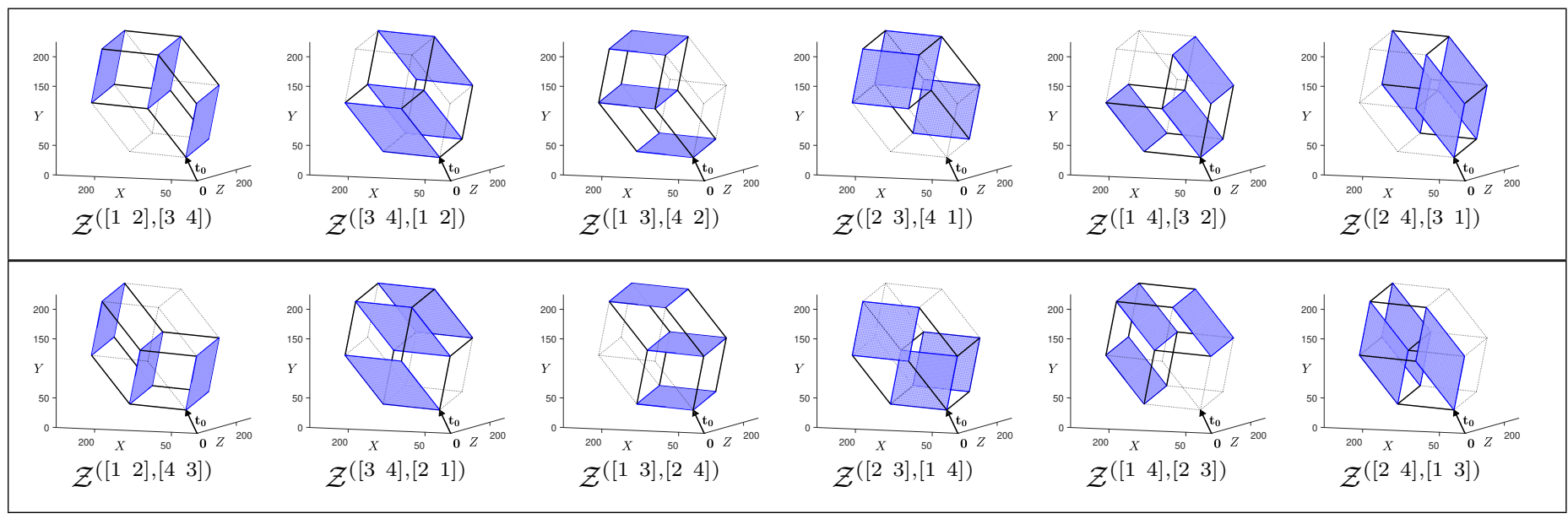

Fig. 6. The collection of complete sets for the four-primary system $\mathbf{P}_{e}^{(4)}$ is comprised of two complete sets delimited by the two boxes. Note that for the complete set $\mathcal{K}$ shown in the top row, the mirror symmetric complete set $\tilde{\mathcal{K}}$ is shown in the bottom row.

is comprised of six facet spans. The facet spans in Figure 6 are organized to highlight the symmetric relationship between the two complete sets: for the complete set $\mathcal{K}$ in the top half, the mirror symmetric complete set $\tilde{\mathcal{K}}=\left\{\mathcal{Z}^{(\mathbb{J}, \tilde{I})} \mid \mathcal{Z}^{(\mathbb{J}, \mathbb{I})} \in \mathcal{K}\right\}$ is shown in the bottom half. In particular, for the facet span $\mathcal{Z}^{(\mathbb{I}, \mathbb{I})} \in \mathcal{K}$, the mirror symmetric facet $\operatorname{span} \mathcal{Z}^{(\mathbb{I}, \tilde{I})} \in \tilde{\mathcal{K}}$ is located below it. From Lemma (4), every facet span belongs to a complete set. In Fig. 6, the symmetry of the complete sets $\mathcal{K}, \tilde{\mathcal{K}}$ implies that $\mathcal{K} \cap \tilde{\mathcal{K}}=\phi$ and the union $\mathcal{K} \bigcup \tilde{\mathcal{K}}$ contains all the facet spans for $\mathbf{P}_{e}^{(4)}$. Therefore, Figure 6 also shows each of the facet spans for $\mathbf{P}_{e}^{(4)}$ without repetition, which can be seen by comparing against Fig. 4. A richer set of examples illustrating all facet spans, all complete sets of compatible facet spans and associated tilings are provided in Section S.I of the Supplementary Materials for five-primary systems that illustrate several aspects that cannot be seen in the four primary case.

As a consequence of Lemma 4, every gamut tiling can be obtained from some maximal set and is uniquely associated with one complete set. We note here that Clause 9 of Theorem 1 in the companion Part I Paper [1] identifies the complete set associated with the corresponding progressive tilings obtained using the methodology developed there. Complete sets provide the link between tilings and the MCS, as we show next.

\section{Gamut Tilings and the vertices of the MCS}

A tiling $\mathcal{T}$ of the gamut $\mathcal{G}$ includes a parallelepiped $\mathcal{P}\left(\mathbf{c}^{\mathbb{P}}, \mathbf{P}[\mathbb{P}]\right) \in \mathcal{T}$ for each $\mathbb{P} \in \mathfrak{C}^{3}(\langle K\rangle)$, where $\mathbf{c}^{\mathbb{P}}$ denotes the origin of the parallelepiped. Now, for any $\mathbb{P} \in \mathfrak{C}^{3}(\langle K\rangle)$, it can be seen that for each $\mathbb{J} \in \mathfrak{C}^{2}(\langle K\rangle), \mathbb{J} \subset \mathbb{P}$, the facet span $\mathcal{Z}^{(\mathbb{J}, \mathbb{I})}$ in the complete set associated with the tiling $\mathcal{T}$, induces the same control vector $\boldsymbol{\alpha}^{\mathbb{P}}$ such that $\mathbf{c}^{\mathbb{P}}=\mathbf{P} \boldsymbol{\alpha}^{\mathbb{P}}+\mathbf{t}_{\mathbf{0}}$ where $\boldsymbol{\alpha}^{\mathbb{P}} \in\{0,1\}^{K}$ and $\alpha_{l}^{\mathbb{P}}=0$, for all $l \in \mathbb{P}$. We obtain the tiling $C C F \mathscr{C}_{\mathcal{T}}$ associated with the tiling $\mathcal{T}$ by defining $\mathscr{C}_{\mathcal{T}}(\mathbf{t})=\boldsymbol{\alpha}^{\mathbb{P}}+\mathbf{I}_{K}[\mathbb{P}] \mathbf{P}^{-1}[\mathbb{P}]\left(\mathbf{t}-\mathbf{c}^{\mathbb{P}}\right)$, for every $\mathbb{P} \in \mathfrak{C}^{3}(\langle K\rangle)$ and every $\mathbf{t} \in \mathcal{P}\left(\mathbf{c}^{\mathbb{P}}, \mathbf{P}[\mathbb{P}]\right)$. The tiling CCF $\mathscr{C}_{\mathcal{T}}$ is continuous over $\mathcal{G}$ and is (piece-wise) linear over each of the parallelepipeds in the tiling $\mathcal{T}$ and uniquely associates $\mathbf{t}$ with the tiling control vector $\mathscr{C}_{T}(\mathbf{t})$. The properties of maximal sets allows us to establish the relationship between gamut tilings, the tiling control vectors and the vertices of the MCS as follows

Theorem 2. For a tristimulus $\mathbf{t} \in \mathcal{G}, \boldsymbol{\omega}$ is a vertex of the MCS $\Omega(\mathbf{t})$ if an only if $\boldsymbol{\omega}$ is a tiling control vector.

One of the examples included in Section S.I of the Supplementary Materials provides all tilings (and associated complete sets) for the $K=5$ primary system $\mathbf{P}_{w}^{(5)}$ that was used in Fig. 2(a). As required by Lemma 2, the clear vertex in Fig. 2(a), which did not have an associated progressive tiling control vector does have an associated tiling control vector among the full set of tilings, which is also identified in Section S.I of the Supplementary Materials.

Theorem 2 characterizes the vertices of the MCS as control vectors obtained from gamut tilings, linking the geometry of MCS with the geometry of gamut representations. To complete this characterization, we highlight how the tilings can be enumerated efficiently.

\section{EFFicient EnUmeration of GAMUt TiLings}

We outline a methodology for efficient enumeration of gamut tilings by exploiting the inter-relations between gamut tilings, complete sets, and maximal sets, and the symmetry properties of facet spans.

\section{A. Maximal Set Construction for Enumeration of Tilings}

From Lemma 4, it can be readily seen that facet spans within a complete set satisfy additional constraints, which we characterize as strong compatibility (s-compatibility). We say that a pair of compatible facet spans $\mathcal{Z}^{(\mathbb{I}, \mathbb{I})}$ and $\mathcal{Z}^{\left(\mathbb{J}^{\prime}, \mathbb{I}^{\prime}\right)}$ are s-compatible if, whenever $l, l^{\prime} \in\langle K-2\rangle$ are such that $(\mathbb{J} \cup \mathbb{I}[l]) \bigcap\left(\mathbb{J}^{\prime} \cup \mathbb{I}^{\prime}\left[l^{\prime}\right]\right)=\overline{\mathbb{J}} \in \mathfrak{C}^{2}(\langle K\rangle)$, i.e., the pair of parallelepipeds $\mathcal{P}_{l}^{(\mathbb{J}, \mathbb{I})} \in \mathcal{Z}^{(\mathbb{J}, \mathbb{I})}$ and $\mathcal{P}_{l^{\prime}}^{\left(\mathbb{J}^{\prime}, \mathbb{I}^{\prime}\right)} \in \mathcal{Z}^{\left(\mathbb{J}^{\prime}, \mathbb{I}^{\prime}\right)}$, are spanned by two common primaries indexed by $\overline{\mathbb{J}}$, then, both $\mathcal{P}_{l}^{(\mathbb{J}, \mathbb{I})}$ and $\mathcal{P}_{l^{\prime}}^{\left(\mathbb{J}^{\prime}, \mathbb{I}^{\prime}\right)}$ are parallelepipeds in a facet span $\mathcal{Z}^{(\overline{\mathrm{J}}, \overline{\mathbb{I}})}$, for 
some $\overline{\mathbb{I}} \in \mathfrak{P}(\langle K\rangle \backslash \overline{\mathbb{J}})$. The following corollary is a then direct consequence of the relation between maximal and complete sets stated in Lemma 4.

Corollary 2. A pair of facets spans in a maximal set are scompatible.

We evaluate s-compatibility between pairs of facet spans to efficiently construct maximal sets, which we use, in turn, for efficiently enumerating the collection of complete sets, using a methodology that we construct in the remainder of this section.

Let $\mathfrak{K}$ be the collection of all complete sets, and let $\mathbb{J}_{1}, \mathbb{J}_{2}, \ldots, \mathbb{J}_{\left(\begin{array}{c}K \\ 2\end{array}\right)}$ be a sequence of all pairs in $\mathfrak{C}^{2}(\langle K\rangle)$. For $j \in\left\langle\left(\begin{array}{c}K \\ 2\end{array}\right)\right\rangle$, let

$$
\begin{aligned}
\mathfrak{A}_{j}=\{ & \left\{\mathcal{Z}^{\left(\mathbb{J}_{1}, \mathbb{I}_{1}\right)}, \ldots, \mathcal{Z}^{\left(\mathbb{J}_{j}, \mathbb{I}_{j}\right)}\right\} \mid \mathbb{I}_{i} \in \mathfrak{P}\left(\langle K\rangle \backslash \mathbb{J}_{i}\right), \text { for all } i \leq j, \\
& \text { and } \left.\mathcal{Z}^{\left(\mathbb{J}_{1}, \mathbb{I}_{1}\right)}, \ldots, \mathcal{Z}^{\left(\mathbb{J}_{j}, \mathbb{I}_{j}\right)} \text { are s-compatible }\right\}
\end{aligned}
$$

be the collection of all sets of $j$ s-compatible facet spans that includes a facet span for each of the index pairs $\mathbb{J}_{1}, \mathbb{J}_{2}, \ldots, \mathbb{J}_{j}$. Thus, $\mathcal{K} \in \mathfrak{A}_{j}$ has cardinality $\mathscr{N}(\mathcal{K})=j$, every $\mathcal{K} \in \mathfrak{A}_{\left(\begin{array}{c}K \\ 2\end{array}\right)}$ is a complete set, and $\mathfrak{A}_{\left(\begin{array}{c}K \\ 2\end{array}\right)}=\mathfrak{K}$.

Observing that $\mathfrak{A}_{1}$ is the set of all facet spans indexed by the index pair $\mathbb{J}_{1}$, the set $\mathfrak{A}_{j+1}$ can be constructed from the set $\mathfrak{A}_{j}$ by identifying for each $\mathcal{K} \in \mathfrak{A}_{j}$, enlargements that add a facet span $\mathcal{Z}^{\left(\mathbb{J}_{j+1}, \mathbb{I}\right)}$ for $\mathbb{I} \in \mathfrak{P}\left(\langle K\rangle \backslash \mathbb{J}_{j+1}\right)$ that is s-compatible with the facet spans already included in $\mathcal{K}$,

$$
\begin{aligned}
\mathfrak{A}_{j+1}=\bigcup_{\mathcal{K} \in \mathfrak{A}_{j}}\left\{\mathcal{K} \cup \mathcal{Z}^{\left(\mathbb{J}_{j+1}, \mathbb{I}\right)} \mid \mathbb{I} \in \mathfrak{P}\left(\langle K\rangle \backslash \mathbb{J}_{j+1}\right)\right. \text { and } \\
\left.\mathcal{Z}^{\left(\mathbb{J}_{j}, \mathbb{I}\right)} \text { is s-compatible with all } \mathcal{Z} \in \mathcal{K}\right\} .
\end{aligned}
$$

Thus, we obtain $\mathfrak{K}$ after $\left(\begin{array}{c}K \\ 2\end{array}\right)$ recursions ${ }^{2}$ of (10). We use the property of maximality to reduce the number of recursions needed to enumerate $\mathfrak{K}$ as follows.

1) Maximal Sets for Enumeration of Tilings: Let $j \in\left\langle\left(\begin{array}{c}K \\ 2\end{array}\right)\right\rangle$. Because any two $\mathcal{K}, \mathcal{K}^{\prime} \in \mathfrak{A}_{j}$ have facet spans for the common index pairs, $\mathbb{J}_{1}, \ldots, \mathbb{J}_{j}$, the parallelepipeds in the associated parallelepiped collections $\mathcal{T}_{\mathcal{K}}$ and $\mathcal{T}_{\mathcal{K}^{\prime}}$ are exactly the ones that are spanned by the primary combinations $\mathbb{P}=\mathbb{J}_{i} \cup k$ for $1 \leq$ $i \leq j$ and $k \in\langle K\rangle \backslash \mathbb{J}_{i}$ and we can see that $\mathscr{N}\left(\mathcal{T}_{\mathcal{K}^{\prime}}\right)=\mathscr{N}\left(\mathcal{T}_{\mathcal{K}}\right)$. It follows that if one element of $\mathfrak{A}_{j}$ is maximal, all elements are maximal. The recursive construction of the sets further ensures that if elements of $\mathfrak{A}_{j}$ are maximal then all elements of $\mathfrak{A}_{m}$ are also maximal for $m \geq j$.

Given the sequence $\mathbb{J}_{1}, \mathbb{J}_{2}, \ldots, \mathbb{J}_{\left(\begin{array}{c}K \\ 2\end{array}\right)}$ of index pairs, let $M$ be the smallest integer $j$ such that the collection of index triples $\bigcup_{i=1}^{j}\left\{\mathbb{J}_{i} \cup k \mid k \in\langle K\rangle \backslash \mathbb{J}_{i}\right\}=\mathfrak{C}^{3}(\langle K\rangle)$. Then, it can be seen that $M$ is the smallest integer $j$ such that $\mathfrak{A}_{j}$ is a collection of maximal sets. Note that $M$ is determined by the specific choice of ordering of the index pairs in the sequence $\mathbb{J}_{1}, \mathbb{J}_{2}, \ldots, \mathbb{J}_{\left(\begin{array}{c}K \\ 2\end{array}\right)}$, so we refer to $M$ as the maximality

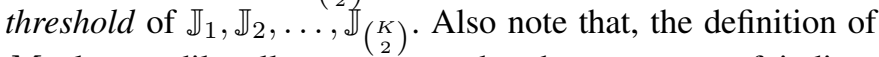
$M$ also readily allows us to order the sequence of indices $\mathbb{J}_{1}, \mathbb{J}_{2}, \ldots, \mathbb{J}_{\left(\begin{array}{l}K \\ 2\end{array}\right)}$ to ensure the smallest possible value for the

\footnotetext{
${ }^{2}$ However, note that the number of operations can vary significantly among the recursions, as $\mathscr{N}\left(\mathfrak{A}_{j}^{\left(\mathbb{J}_{1}, \mathbb{I}\right)}\right)$ varies with $j$.
}

maximality threshold $M$. Example orderings that minimize the maximality threshold $M$ are provided in Table II for $K=$ $3,4, \ldots 9$.

Let $\mathcal{A}_{M}(\cdot) \quad: \quad \mathfrak{K} \rightarrow \mathfrak{A}_{M}$ be the function defined for every complete set $\mathcal{K} \in \mathfrak{K}$ as $\mathcal{A}_{M}(\mathcal{K})=$ $\left\{\mathcal{Z}^{\left(\mathbb{J}_{j}, \mathbb{I}_{j}\right)} \mid \mathcal{Z}^{\left(\mathbb{J}_{j}, \mathbb{I}_{j}\right)} \in \mathcal{K}, j \in\langle M\rangle\right\}$, i.e., the set of the $M$ facet spans of $\mathcal{K}$ corresponding to $\mathbb{J}_{1}, \mathbb{J}_{2}, \ldots \mathbb{J}_{M}$. Recalling from Lemma 4 that a maximal set is a subset of one and only one complete set, we see that for $\mathcal{K}, \mathcal{K}^{\prime} \in \mathfrak{K}, \mathcal{A}_{M}(\mathcal{K})=\mathcal{A}_{M}\left(\mathcal{K}^{\prime}\right)$ implies $\mathcal{K}=\mathcal{K}^{\prime}$, and that every element of $\mathfrak{A}_{M}$ is a set of the first $M$ facet spans of one complete set, therefore, $\mathcal{A}_{M}(\cdot)$ is one-to-one and onto, thus, a bijection between $\mathfrak{A}_{M}$ and $\mathfrak{K}$, so $\mathscr{N}\left(\mathfrak{A}_{M}\right)=\mathscr{N}(\mathfrak{K})$, therefore, enumerating $\mathfrak{K}$ is equivalent to enumerating $\mathfrak{A}_{M}$, which we obtain with $M$ recursions of (10).

Next, we introduce a partition of $\mathfrak{A}_{M}$ into disjoint collections of maximal sets that allows us to exploit the properties of symmetry of facet spans to reduce by half the number of operations for the construction of $\mathfrak{A}_{M}$ and subsequent enumeration of gamut tilings

2) Partitioning of Complete/Maximal Sets: Let $\mathbb{I}_{1}, \ldots, \mathbb{I}_{(K-2) \text { ! }}$ be an enumeration of the elements of $\mathfrak{P}\left(\langle K\rangle \backslash \mathbb{J}_{1}\right)$ such that $\mathbb{I}_{i+(K-2) ! / 2}=\tilde{\mathbb{I}}_{i}$. Because every facet span indexed by $\mathbb{J}_{1}$ belongs to a complete set, and every complete set includes only one facet span indexed by the index pair $\mathbb{J}_{1}$, the collection of complete sets $\mathfrak{K}$ is a disjoint union, $\mathfrak{K}=\bigcup_{i=1}^{(K-2) !} \mathfrak{K}^{\left(\mathbb{J}_{1}, \mathbb{I}_{i}\right)}$, where $\mathfrak{K}^{\left(\mathbb{J}_{1}, \mathbb{I}_{i}\right)}$ is the set of all complete sets containing the facet span $\mathcal{Z}^{\left(\mathbb{J}_{1}, \mathbb{I}_{i}\right)}$. Recalling that $\mathcal{A}_{M}(\cdot)$ is a bijection between $\mathfrak{A}_{M}$ and $\mathfrak{K}$, we express $\mathfrak{A}_{M}=\mathcal{A}_{M}(\mathfrak{K})=\bigcup_{i=1}^{(K-2) !} \mathcal{A}_{M}\left(\mathfrak{K}^{\left(\mathbb{J}_{1}, \mathbb{I}_{i}\right)}\right)=\bigcup_{i=1}^{(K-2) !} \mathfrak{A}_{M}^{\left(\mathbb{J}_{1}, \mathbb{I}_{i}\right)}$, where $\mathfrak{A}_{j}^{\left(\mathbb{J}_{1}, \mathbb{I}\right)}=\left\{\mathcal{K} \in \mathfrak{A}_{j} \mid \mathcal{Z}^{\left(\mathbb{I}_{1}, \mathbb{I}\right)} \in \mathcal{K}\right\}$ is the collection of all sets of $j$ s-compatible facet spans that include $\mathcal{Z}^{\left(\mathbb{J}_{1}, \mathbb{I}\right)}$ and a facet span for each of the index pairs $\mathbb{J}_{2}, \ldots, \mathbb{J}_{j}$.

From Lemma 4, we see that $\mathfrak{A}^{\left(\mathbb{J}_{1}, \mathbb{I}_{i+(K-2) ! / 2}\right)}=\mathfrak{A}^{\left(\mathbb{J}_{1}, \tilde{\mathbb{I}}_{i}\right)}$ and $\mathscr{N}\left(\mathfrak{A}^{\left(\mathbb{J}_{1}, \tilde{\mathbb{I}}_{i}\right)}\right)=\mathscr{N}\left(\mathfrak{A}^{\left(\mathbb{I}_{1}, \mathbb{I}_{i}\right)}\right)$, therefore,

$$
\mathfrak{A}_{M}=\bigcup_{i=1}^{(K-2) ! / 2} \mathfrak{A}_{M}^{\left(\mathbb{J}_{1}, \mathbb{I}_{i}\right)} \cup \mathfrak{A}_{M}^{\left(\mathbb{J}_{1}, \tilde{\mathbb{I}}_{i}\right)},
$$

and

$$
\mathscr{N}(\mathfrak{K})=\mathscr{N}\left(\mathfrak{A}_{M}\right)=2 \sum_{i=1}^{(K-2) ! / 2} \mathscr{N}\left(\mathfrak{A}_{M}^{\left(\mathbb{J}_{1}, \mathbb{I}_{i}\right)}\right) .
$$

And analogous to $\mathfrak{A}_{j}$, for $\mathbb{I} \in \mathfrak{P}\left(\langle K\rangle \backslash \mathbb{J}_{1}\right)$, the collection $\mathfrak{A}_{j}^{\left(\mathbb{J}_{1}, \mathbb{I}_{i}\right)}$ for $j=1,2, \ldots M$ can be recursively constructed as $\mathfrak{A}_{1}^{\left(\mathbb{J}_{1}, \mathbb{I}_{i}\right)}=\left\{\mathcal{Z}^{\left(\mathbb{J}_{1}, \mathbb{I}_{i}\right)}\right\}$, and

$$
\begin{aligned}
\mathfrak{A}_{j+1}^{\left(\mathbb{J}_{1}, \mathbb{I}_{i}\right)}= & \bigcup_{\mathcal{K} \in \mathfrak{A}_{j}^{\left(\mathbb{J}_{1}, \mathbb{I}_{i}\right)}}\left\{\mathcal{K} \cup \mathcal{Z}^{\left(\mathbb{J}_{j+1}, \mathbb{I}\right)} \mid \mathbb{I} \in \mathfrak{P}\left(\langle K\rangle \backslash \mathbb{J}_{j+1}\right),\right. \text { and } \\
& \left.\mathcal{Z}^{\left(\mathbb{J}_{j+1}, \mathbb{I}\right)} \text { is s-compatible with all } \mathcal{Z} \in \mathcal{K}\right\} .
\end{aligned}
$$

The algorithmic description for building the collections of maximal sets using the recursion in (13) is described next.

3) Algorithmic Enumeration of Gamut Tilings: Let $\mathbb{J}_{1}, \mathbb{J}_{2}, \ldots, \mathbb{J}_{\left(\begin{array}{c}K \\ 2\end{array}\right)} \in \mathfrak{C}^{2}(\langle K\rangle)$ be a sequence index pairs with the smallest maximality threshold $M$, let $\mathbb{I}_{1}, \ldots, \mathbb{I}_{(K-2)}$ ! be a sequence of all the permutations in $\mathfrak{P}\left(\langle K\rangle \backslash \mathbb{J}_{1}\right)$ such that 


\begin{tabular}{|c|c|c|c|c|c|c|c|c|c|c|c|c|c|c|c|c|c|}
\hline$K$ & $M$ & $\mathbb{J}_{1}$ & $\mathbb{J}_{2}$ & $\mathbb{J}_{3}$ & $\mathbb{J}_{4}$ & $\mathbb{J}_{5}$ & $\mathbb{J}_{6}$ & $\mathbb{J}_{7}$ & $\mathbb{J}_{8}$ & $\mathbb{J}_{9}$ & $\mathbb{J}_{10}$ & $\mathbb{J}_{11}$ & $\mathbb{J}_{12}$ & $\mathbb{J}_{13}$ & $\mathbb{J}_{14}$ & $\mathbb{J}_{15}$ & $\mathbb{J}_{16}$ \\
\hline 3 & 1 & {$\left[\begin{array}{ll}1 & 2\end{array}\right]$} & - & - & - & - & - & - & - & - & - & - & - & - & - & - & - \\
\hline 4 & 2 & {$\left[\begin{array}{ll}1 & 2\end{array}\right]$} & {$\left[\begin{array}{ll}3 & 4\end{array}\right]$} & - & - & - & - & - & - & - & - & - & - & - & - & - & - \\
\hline 5 & 4 & {$\left[\begin{array}{ll}1 & 2\end{array}\right]$} & {$\left[\begin{array}{ll}3 & 4\end{array}\right]$} & {$\left[\begin{array}{ll}1 & 5\end{array}\right]$} & {$\left[\begin{array}{ll}2 & 5\end{array}\right]$} & - & - & - & - & - & - & - & - & - & - & - & - \\
\hline 6 & 6 & {$\left[\begin{array}{ll}1 & 2\end{array}\right]$} & {$\left[\begin{array}{ll}3 & 4\end{array}\right]$} & {$\left[\begin{array}{ll}1 & 5\end{array}\right]$} & {$\left[\begin{array}{ll}2 & 5\end{array}\right]$} & {$\left[\begin{array}{ll}3 & 6\end{array}\right]$} & {$\left[\begin{array}{ll}4 & 6\end{array}\right]$} & - & - & - & - & - & - & - & - & - & - \\
\hline 7 & 9 & {$\left[\begin{array}{ll}1 & 2\end{array}\right]$} & {$\left[\begin{array}{ll}3 & 4\end{array}\right]$} & {$\left[\begin{array}{ll}5 & 6\end{array}\right]$} & {$\left[\begin{array}{ll}1 & 7\end{array}\right]$} & {$\left[\begin{array}{ll}2 & 7\end{array}\right]$} & {$\left[\begin{array}{ll}3 & 5\end{array}\right]$} & {$\left[\begin{array}{ll}4 & 6\end{array}\right]$} & {$\left[\begin{array}{ll}3 & 6\end{array}\right]$} & {$\left[\begin{array}{ll}4 & 5\end{array}\right]$} & - & - & - & - & - & - & - \\
\hline 8 & 12 & {$\left[\begin{array}{ll}1 & 2\end{array}\right]$} & {$\left[\begin{array}{ll}3 & 4\end{array}\right]$} & {$\left[\begin{array}{ll}5 & 6\end{array}\right]$} & {$\left[\begin{array}{ll}7 & 8\end{array}\right]$} & {$\left[\begin{array}{ll}1 & 3\end{array}\right]$} & {$\left[\begin{array}{ll}2 & 4\end{array}\right]$} & {$\left[\begin{array}{ll}5 & 7\end{array}\right]$} & {$\left[\begin{array}{ll}6 & 8\end{array}\right]$} & {$\left[\begin{array}{ll}2 & 3\end{array}\right]$} & {$\left[\begin{array}{ll}1 & 4\end{array}\right]$} & {$\left[\begin{array}{ll}5 & 8\end{array}\right]$} & {$\left[\begin{array}{ll}6 & 7\end{array}\right]$} & - & - & - & - \\
\hline 9 & 16 & {$\left[\begin{array}{ll}1 & 2\end{array}\right]$} & {$\left[\begin{array}{ll}3 & 4\end{array}\right]$} & {$\left[\begin{array}{ll}5 & 6\end{array}\right]$} & {$\left[\begin{array}{ll}7 & 8\end{array}\right]$} & {$\left[\begin{array}{ll}1 & 9\end{array}\right]$} & {$\left[\begin{array}{ll}2 & 9\end{array}\right]$} & {$\left[\begin{array}{ll}3 & 5\end{array}\right]$} & {$\left[\begin{array}{ll}3 & 6\end{array}\right]$} & {$\left[\begin{array}{ll}4 & 6\end{array}\right]$} & {$\left[\begin{array}{ll}4 & 5\end{array}\right]$} & {$\left[\begin{array}{ll}1 & 7\end{array}\right]$} & {$\left[\begin{array}{ll}8 & 9\end{array}\right]$} & {$\left[\begin{array}{ll}1 & 8\end{array}\right]$} & {$\left[\begin{array}{ll}2 & 7\end{array}\right]$} & {$\left[\begin{array}{ll}2 & 8\end{array}\right]$} & {$\left[\begin{array}{ll}7 & 9\end{array}\right]$} \\
\hline
\end{tabular}

TABLE II

EXAMPLE INDEX ORDERINGS $\mathbb{J}_{1}, \ldots, \mathbb{J}_{M}$ THAT MINIMIZE THE MAXIMALITY THRESHOLD $M$ FOR $K=3,4, \ldots 9$.

$\mathbb{I}_{i+(K-2) ! / 2}=\tilde{\mathbb{I}}_{i}$. Taking as inputs $\mathbb{J}_{1}, \mathbb{J}_{2}, \ldots, \mathbb{J}_{M}$, and $\mathbb{I}_{i}$, with $i \in\langle(K-2) ! / 2\rangle$, and via a depth-first search methodology described in Algorithm 1, we obtain $m_{i}=\mathscr{N}\left(\mathfrak{A}_{M}^{\left(\mathbb{J}_{1}, \mathbb{I}_{i}\right)}\right)$ and a sequence of maximal sets $\mathcal{M}_{1}^{\left(\mathbb{J}_{1}, \mathbb{I}_{i}\right)}, \ldots, \mathcal{M}_{m_{i}}^{\left(\mathbb{J}_{1}, \mathbb{I}_{i}\right)}$ enumerating $\mathfrak{A}_{M}^{\left(\mathbb{J}_{1}, \mathbb{I}_{i}\right)}$.

The depth-first search strategy is implemented with a stack of s-compatible facet spans $\mathcal{K}_{1}, \ldots, \mathcal{K}_{n}, n \geq 0$, where the stack is initialized with $\mathcal{K}_{1}=\left\{\mathcal{Z}^{\left(\mathbb{J}_{1}, \mathbb{I}_{i}\right)}\right\}$. In the depthfirst search, the set $\mathcal{K}=\mathcal{K}_{n}$ is retrieved from the stack; $\mathcal{K} \in \mathfrak{A}_{j}^{\left(\mathbb{J}_{1}, \mathbb{I}_{i}\right)}$ where $j=\mathscr{N}(\mathcal{K})$; and we search and push into the stack, all the s-compatible sets resulting from enlarging $\mathcal{K}$ by a facet span indexed by $\mathbb{J}_{j+1}$. The process is repeated until the extensions become maximal and elements of $\mathfrak{A}_{M}^{\left(\mathbb{J}_{1}, \mathbb{I}_{i}\right)}$, and thus, part of the of the sequence of maximal sets $\mathcal{M}_{1}^{\left(\mathbb{J}_{1}, \mathbb{I}_{i}\right)}, \ldots, \mathcal{M}_{m_{i}}^{\left(\mathbb{J}_{1}, \mathbb{I}_{i}\right)}$.

We execute Algorithm 1 for all $i \in\langle(K-2) ! / 2\rangle$, thus enumerating $\mathfrak{A}_{M}^{\left(\mathbb{J}_{1}, \mathbb{I}_{i}\right)}$, and by the properties of symmetry, enumerating $\mathfrak{A}_{M}^{\left(\mathbb{J}_{1}, \widetilde{\mathbb{I}}_{i}\right)}$, which allows us to enumerate the collection of complete sets $\mathfrak{K}$ and all gamut tilings, and from (12), to conclude that $\mathscr{N}(\mathfrak{K})=2 \sum_{i}^{(K-2) ! / 2} m_{i}$.

The time efficiency of the enumeration process can be further improved by observing that: (a) the proposed methodology is suitable for parallelization because the computation of $\mathfrak{A}_{M}^{\left(\mathbb{J}_{1}, \mathbb{I}_{i}\right)}$ is independent for every $i \in\langle(K-2) ! / 2\rangle$, (b) checking whether a pair of parallelepipeds intersect each other, a fundamental and time-demanding step in the evaluation of scompatibility in Algorithm 2, can be efficiently implemented using a methodology that exploits the gamut representation introduced in the companion Part I paper [1], as detailed in Appendix E, and (c) a significant reduction of repetitive operations is achieved by pre-computing the s-compatibility between the pairs of facet spans, which in-turn, benefits from pre-computing the s-compatibility between all possible parallelepiped pairs. The gains in time are at the cost of increased memory requirements. Because, at any iteration of Algorithm 2, there are at most $(K-2)$ ! enlargements of $\mathcal{K}=\mathcal{K}_{n}$ by one facet span, and $\mathcal{K}$ admits at most $(M-2)$ additional facet spans to produce a s-compatible set that is not maximal, the depth-search methodology guarantees that the stack has at most $(M-2)(K-2)$ ! elements.

\section{B. Tiling Enumerations for Some Primary Configurations}

We use our methodology to enumerate the gamut tilings for some primary configurations. We first enumerate gamut tilings

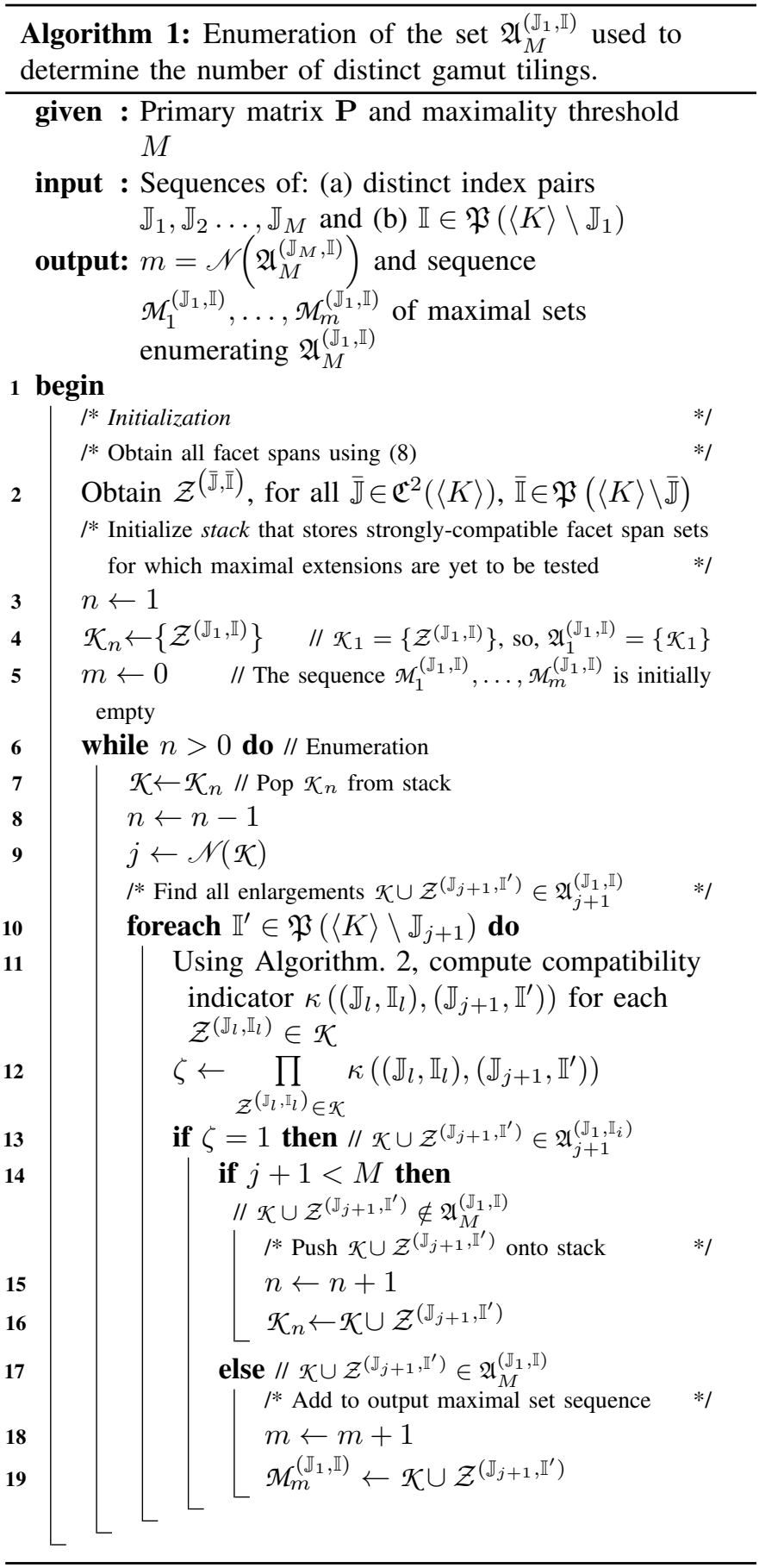




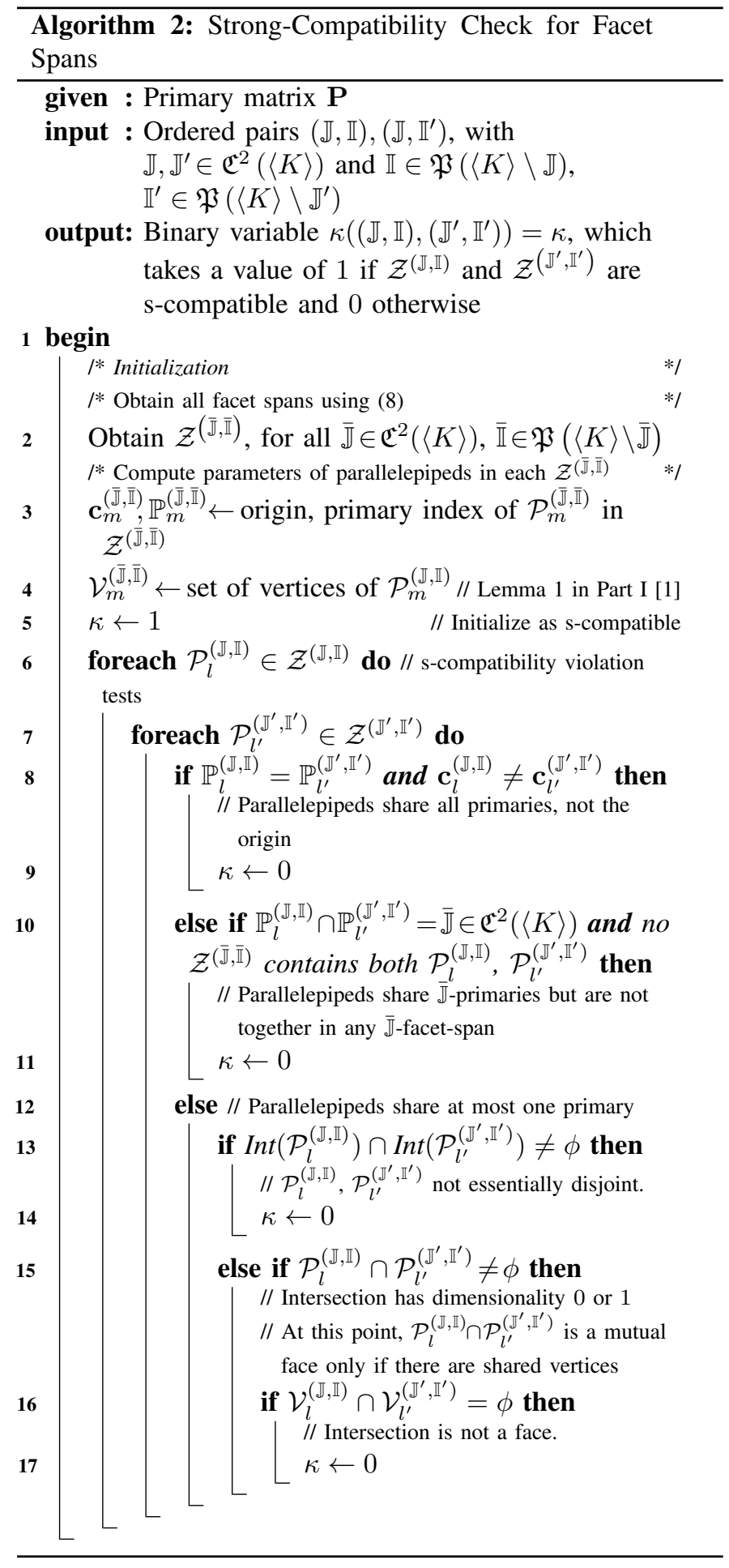

for families of primary configurations where each primary enlarges the chromaticity gamut [8, pp. 137]. For this situation, the chromaticity of each primary is a vertex of the chromaticity gamut and, for a given $K$, all primary configurations satisfying the stated requirement have the same number of gamut tilings, which we present for $K=3,4,5, \ldots 9$ in Table III. The number of tilings for the primary configurations under consideration match the number of tilings for polar zonohedra [9]. The enumerations of polar zonohedra tilings in [10], provides validation of our results for $K=4,5,6$, and 7 , and, vice versa, our results expand the enumeration of tilings for the polar zonohedra for the cases of $K=8$ and 9 . In Section S.II of the Supplementary Materials, we detail the computation time required for obtaining these enumerations and also highlight the speed-up resulting from the efficient enumeration approach developed in Section V. We note that the computational requirements for enumerating the tilings grow rapidly with increasing $K$ and obtaining the number of tilings for $K>9$ is therefore challenging.

Although a full discussion is beyond the scope of the present paper, we note that three-dimensional zonotopes have been categorized into classes based on the interconnection structure of their vertices represented in projective diagrams [11], where the zonotopes in a given class are known to have the same number of gamut tilings [10]. Our methodology for enumerating the tilings can also be used to enumerate the number of tilings for each of these classes under the constraint that the zonotope is obtained as the linear (or affine) mapping of $[0,1]^{K}$ by a $3 \times K$ matrix $\mathbf{A}$ such that any three columns of $\mathbf{A}$ are linearly independent. Table IV enumerates the number of tilings for alternative classes of for $K=3,4,5,6,7$, where the polar configuration results can be seen as one of the specific subclasses.

\begin{tabular}{rccccccc}
\hline$K=$ & 3 & 4 & 5 & 6 & 7 & 8 & 9 \\
\hline $\mathscr{N}(\mathfrak{K})=$ & 1 & 2 & 10 & 148 & 7686 & 1681104 & 1881850464 \\
\hline \multicolumn{7}{c}{ TABLE III }
\end{tabular}

NUMBER OF TILINGS $(\mathscr{N}(\mathfrak{K}))$ FOR GAMUTS WITH PRIMARIES THAT INDIVIDUALLY ENLARGE THE CHROMATICITY GAMUT ( $\equiv$ POLAR CONFIGURATION)

\section{DISCUSSION}

As result of the characterization of the vertices of the MCS as tiling control vectors in Theorem 2, different properties of the gamut and control vectors emerge naturally from our framework with a geometric interpretation. For instance, the uniqueness of control vectors for points on the gamut boundary, which is stated and proved in the companion Part I paper [1] and in [12], can also be seen as the consequence of the fact that all complete sets share the same boundary representation, therefore, all tiling CCFs match on the boundary and the MCS is a singleton set for all tristimuli on the boundary.

The enumeration of all tiling CCFs follows immediately from the enumeration of the gamut tilings presented in Section V. Because of the convexity of the MCS, we see that every $\mathrm{CCF} \mathscr{C}$ can be express at $\mathbf{t} \in \mathcal{G}$ as a convex combination of the 


\begin{tabular}{|c|c|c|c|c|c|c|c|}
\hline \multirow[t]{2}{*}{$K$} & \multirow[t]{2}{*}{ Class } & \multicolumn{5}{|c|}{ N. of vertices with valency $v$} & \multirow[t]{2}{*}{$\mathscr{N}(\mathfrak{K})$} \\
\hline & & $v=3$ & 4 & 5 & 6 & 7 & \\
\hline 3 & 1 & 8 & - & - & - & - & 1 \\
\hline 4 & 1 & 8 & 6 & - & - & - & 2 \\
\hline 5 & 1 & 10 & 10 & 2 & - & - & 10 \\
\hline \multirow[t]{4}{*}{6} & 1 & 12 & 16 & 4 & 0 & - & 144 \\
\hline & 2 & 12 & 18 & 0 & 2 & - & 148 \\
\hline & 3 & 14 & 12 & 6 & 0 & - & 148 \\
\hline & 4 & 20 & 0 & 12 & 0 & - & 160 \\
\hline \multirow[t]{11}{*}{7} & $1 a$ & 14 & 24 & 6 & 0 & 0 & 6902 \\
\hline & $1 b$ & 14 & 24 & 6 & 0 & 0 & 6932 \\
\hline & 2 & 14 & 26 & 2 & 2 & 0 & 7220 \\
\hline & 3 & 14 & 28 & 0 & 0 & 2 & 7686 \\
\hline & $4 a$ & 16 & 20 & 8 & 0 & 0 & 7106 \\
\hline & $4 b$ & 16 & 20 & 8 & 0 & 0 & 7242 \\
\hline & 5 & 16 & 22 & 4 & 2 & 0 & 7468 \\
\hline & 6 & 18 & 16 & 10 & 0 & 0 & 7518 \\
\hline & 7 & 18 & 18 & 6 & 2 & 0 & 7624 \\
\hline & 8 & 20 & 12 & 12 & 0 & 0 & 7690 \\
\hline & 9 & 22 & 10 & 10 & 2 & 0 & 8260 \\
\hline
\end{tabular}

TABLE IV

NUMBER OF TILINGS $\mathscr{N}(\mathfrak{K})$ FOR ALL POSSIBLE THREE DIMENSIONAL ZONOTOPE CLASSES GENERATED BY A $3 \times K$ MATRIX A SUCH THAT ANY THREE COLUMNS ARE LINEARLY INDEPENDENT FOR $K=3,4, \ldots 7$.

tiling control vectors of $\mathbf{t}$, i.e., $\mathscr{C}(\mathbf{t})=\sum_{\mathcal{K} \in \mathfrak{K}} \zeta_{\mathcal{K}}(\mathbf{t}) \mathscr{C}_{I_{\mathcal{K}}}(\mathbf{t})$, with $\sum_{\mathcal{K} \in \mathfrak{K}} \zeta_{\mathcal{K}}(\mathbf{t})=1$, where $\zeta_{\mathcal{K}}: \mathcal{G} \rightarrow[0,1]$ is a weighting function associated to the tiling $\mathcal{T}_{\mathcal{K}}$. This representation characterization can potentially be helpful for CCF analysis and design.

Our framework can also support, complement, and provide additional insight into existing approaches for color control selection and CCF design. The vertices of the MCS obtained using our proposed approach can be used to compute and visualize the centroid of the MCS, which has been proposed as a method for selecting control values for driving multiprimary displays [13]. The characterization of the MCS also facilitates the design of CCFs to optimize a variety of objective functions modeling display performance using the projected gradient descent algorithm [14], [15]. This approach was introduced in [16], where it was used to obtain an optimal CCF that is perceptually robust to primary variations. When the objective function is linear, the optimal control vectors, which are solutions to linear-programming problems over the MCS, correspond to vertices of the MCS that are characterized algebraically by Theorem 1 and geometrically by Theorem 2 as tiling control vectors. An important subcase of practical interest is the design of power consumption minimizing CCFs for display technologies, such as organic light emitting diodes (OLEDs), where the power can be modeled as a linear function of the control vectors [17]. The characterization for control vectors for optimal power in terms of gamut tilings was introduced independently and near concurrently in [4], [18].

The orthogonal decomposition and visualization strategy presented in Section III-A can be used as a tool for supporting the selection of control vectors, showing the flexibility for color control through visualizations of the MCS. It can also be used to visualize and compare alternative CCFs along specific regions of the gamut, as used in [16] for visualizing the smoothness of CCFs, a criteria that has motivated several methodologies for CCF design [13], [19]-[21], and is an important feature of robustness to primary variations [22].

The concept of a facet span is the fundamental building block of the framework introduced for characterizing gamut tilings, and builds upon the infrastructure presented in the companion Part I paper [1] for characterizing the gamut. Because the characterization of the gamut boundary (Clause 3, Theorem 1 in Part I) is applicable for any three dimensional zonotope, where $\mathbf{P}$ is a $3 \times K$ matrix for which any three columns are linearly independent, the definition of facet span, and by extension, our characterization of tilings are also applicable to the aforementioned broader class of zonotopes. Characterizations of zonotopal tilings have been previously proposed [2, Chap. 7], and the tilings for particular classes of zonotopes have been studied [23]-[26], however, enumerations of zonotopal tilings remains an open problem. The computational enumeration results for $K \leq 9$ presented in Table III extend those obtained for polar zonotopes for $K \leq 7$ in [10]. The results in Table IV also enumerate the possible number of tilings for all three dimensional zonotopes with $K \leq 8$ where any three columns of the matrix $\mathbf{P}$ are linearly independent.

\section{CONCLUSION}

The mathematical results we develop and present provide a complete characterization of MCS for multiprimary displays by relating the MCS to (parallelepiped) gamut tilings of the gamut. Specifically, we show that the vertices of the MCS polytope correspond exactly to the control vectors obtained from $\mathrm{CCFs}$ associated with (parallelepiped) tilings of the gamut. Our results provide, not only a theoretical framework interlinking the geometry of the tristimulus gamut with the geometry of the MCS, but also connect to applications in color reproduction, providing insight into alternative strategies that have been proposed for multiprimary display color control. Additionally, the characterizations of tilings and the efficient enumeration methodology that we provide also applies broadly to zonotopes in $\mathbb{R}^{3}$ whose generating line segments satisfy the mild constraint that any subset of three is linearly independent. Computational results obtained using the proposed approach also extend known results on enumeration of tilings for zonotopes in $\mathbb{R}^{3}$. This paper and the companion Part I paper offer a unified and comprehensive framework for the characterization of the gamut and color control for multiprimary display design, modeling, and color management.

\section{ACKNOWLEDGMENT}

We thank the Center for Integrated Research Computing, University of Rochester, for providing access to computational resources.

\section{APPENDIX A} ProOF OF THEOREM 1

Let $\mathbf{t} \in \mathcal{G}, \boldsymbol{\omega}=\left[\omega_{1}, \ldots, \omega_{K}\right]^{T} \in \Omega(\mathbf{t})$. For $K=3$ the theorem holds trivially. Assume $K \geq 4$, let $m$, with $0 \leq m \leq K$, be the number of vector components of $\boldsymbol{\omega}$ with values in $(0,1)$, and assume without loss of generality that said vector components correspond to the first $m$ entries of $\boldsymbol{\omega}$ (otherwise, simply 
re-order the primaries in $\mathbf{P}$ ). We shall prove the contrapositive statement: $m>3$ iff $\boldsymbol{\omega}$ is not a vertex of $\Omega(\mathbf{t})$. Assume first that $m>3$. Because $\mathbf{p}_{1}, \ldots, \mathbf{p}_{m}$ are linearly dependent, there exist $\nu_{1}, \ldots, \nu_{m} \in \mathbb{R}$, not all zero, such that $\sum_{k=1}^{m} \nu_{k} \mathbf{p}_{k}=\mathbf{0}$. So let $\boldsymbol{\nu}=\left[\nu_{1}, \nu_{2}, \ldots, \nu_{m}, \nu_{m+1}, \ldots, \nu_{K}\right]^{T}$, with $\nu_{k}=0$ for $k>m$, and let $\delta=\min \left\{\omega_{k}, 1-\omega_{k} \mid k \in\langle m\rangle\right\}$. Then $\delta>0$ and $\left(\omega_{k}+\delta\right),\left(\omega_{k}-\delta\right) \in[0,1]$, for all $k \in\langle m\rangle$. Observing that $\boldsymbol{\nu} \neq \mathbf{0}$ and $\delta>0$, we see that the vectors $\boldsymbol{\omega}^{-}=\boldsymbol{\omega}-\delta \boldsymbol{\nu} /\|\boldsymbol{\nu}\|$ and $\boldsymbol{\omega}^{+}=\boldsymbol{\omega}+\delta \boldsymbol{\nu} /\|\boldsymbol{\nu}\|$, are different from each other, different from $\boldsymbol{\omega}$, and are inside $[0,1]^{K}$. Because $\mathbf{P} \boldsymbol{\nu}=\mathbf{0}$, we see that $\mathbf{P} \boldsymbol{\omega}^{-}=\mathbf{P} \boldsymbol{\omega}^{+}=\mathbf{P} \boldsymbol{\omega}$, therefore, $\boldsymbol{\omega}^{-}, \boldsymbol{\omega}^{+} \in \Omega(\mathbf{t})$. Observing that $\boldsymbol{\omega}=(1 / 2)\left(\boldsymbol{\omega}^{-}+\boldsymbol{\omega}^{+}\right)$, we conclude that $\boldsymbol{\omega}$ is the convex combination of two different vectors in $\Omega(\mathbf{t})$, therefore $\boldsymbol{\omega}$ is not a vertex of $\Omega(\mathbf{t})$.

To show the converse, assume now that $\boldsymbol{\omega}$ is not a vertex of $\Omega(\mathbf{t})$, so $\boldsymbol{\omega}$ is the convex combination of two different control vectors $\boldsymbol{\alpha}, \boldsymbol{\gamma} \in \Omega(\mathbf{t})$, so $\boldsymbol{\omega}=\zeta \boldsymbol{\alpha}+(1-\zeta) \boldsymbol{\gamma}$, for some $\zeta \in$ $(0,1)$. Let $\boldsymbol{\nu}=\boldsymbol{\alpha}-\boldsymbol{\gamma}$. Recalling that $\alpha_{k}, \gamma_{k} \in[0,1]$, we see for $k>m$ that $\alpha_{k}=\gamma_{k}=\omega_{k} \in\{0,1\}$, so $\nu_{k}=0$, and because $\boldsymbol{\alpha} \neq \boldsymbol{\gamma}$, there is some $k \leq m$ such that $\nu_{k} \neq 0$. Observing that $\sum_{k=1}^{m} \nu_{k} \mathbf{p}_{k}=\mathbf{P} \boldsymbol{\nu}=\mathbf{0}$, we see that $\mathbf{p}_{1}, \ldots, \mathbf{p}_{m}$ are linearly dependent, and because every set of three (or fewer) primaries is linearly independent, we conclude that $m>3$.

\section{APPENDIX B \\ Proofs For Section IV Lemmas AND Theorem}

\section{A. Proof of Lemma 1}

Obviously, $\mathbb{I}=\mathbb{I}^{\prime}$ implies $\mathcal{Z}^{(\mathbb{J}, \mathbb{I})}=\mathcal{Z}^{\left(\mathbb{J}, \mathbb{I}^{\prime}\right)}$. We prove the converse by showing its contrapositive statement: $\mathbb{I} \neq \mathbb{I}^{\prime}$ implies $\mathcal{Z}^{(\mathbb{J}, \mathbb{I})} \neq \mathcal{Z}^{\left(\mathbb{J}, \mathbb{I}^{\prime}\right)}$. Assume $\mathbb{I} \neq \mathbb{I}^{\prime}$, and let $l$ be the smallest integer such that $\mathbb{I}[l] \neq \mathbb{I}^{\prime}[l]$ (note, $l \geq 1$ ). We see from (7) that $\mathbf{d}_{l-1}^{\mathbb{J}+}(\mathbb{I})=\mathbf{d}_{l-1}^{\mathbb{J}+}\left(\mathbb{I}^{\prime}\right)$ indicating that $\mathcal{F}_{l-1}^{(\mathbb{J}, \mathbb{I})}=\mathcal{F}_{l-1}^{\left(\mathbb{J}, \mathbb{I}^{\prime}\right)}$ is a common facet for $\mathcal{P}_{l}^{(\mathbb{I}, \mathbb{I})}$ and $\mathcal{P}_{l}^{\left(\mathbb{J}, \mathbb{I}^{\prime}\right)}$. Assume, without loss of generality, that $\mathbf{u}_{\mathbb{J}}^{T} \mathbf{d}_{l}^{\mathbb{J}+}(\mathbb{I}) \leq \mathbf{u}_{\mathbb{J}}^{T} \mathbf{d}_{l}^{\mathbb{J}+}\left(\mathbb{I}^{\prime}\right)$ (otherwise simply swap $\mathbb{I}$ and $\left.\mathbb{I}^{\prime}\right)$. Consider the vertices $\mathbf{v}_{1}=\mathbf{d}_{l}^{\mathbb{J}+}(\mathbb{I})=$ $\mathbf{d}_{l-1}^{\mathbb{J}+}\left(\mathbb{I}^{\prime}\right)+\operatorname{sgn}\left(\mathbf{u}_{\mathbb{J}}^{T} \mathbf{p}_{\mathbb{I}[l]}\right) \mathbf{p}_{\mathbb{I}[l]}$ and $\mathbf{v}_{2}=\mathbf{v}_{1}+\mathbf{p}_{\mathbb{J}[1]}+\mathbf{p}_{\mathbb{I}[2]}$ of the facet $\mathcal{F}_{l}^{(\mathbb{J}, \mathbb{I})}$ We show that at least one of $\mathbf{v}_{1}$ or $\mathbf{v}_{2}$ is not contained in $\mathcal{P}_{l}^{\left(\mathbb{J}, \mathbb{I}^{\prime}\right)}$ and $\mathcal{Z}^{\left(\mathbb{J}, \mathbb{I}^{\prime}\right)}$, a situation that is illustrated in Fig. 7. Because $\mathbf{P}\left[\mathbb{J} \mathbb{I}^{\prime}[l]\right]$ is full rank, there exists a unique $\boldsymbol{\nu} \in \mathbb{R}^{3}$ such that $\mathbf{v}_{1}=\mathbf{c}_{l}^{\left(\mathbb{J}, \mathbb{I}^{\prime}\right)}+\mathbf{P}\left[\mathbb{J} \mathbb{I}^{\prime}[l]\right] \boldsymbol{\nu}$, whereby $\mathbf{v}_{2}=\mathbf{v}_{1}+\mathbf{p}_{\mathbb{J}[1]}+\mathbf{p}_{\mathbb{J}[2]}=\mathbf{c}_{l}^{\left(\mathbb{J} \mathbb{I}^{\prime}\right)}+\mathbf{P}\left[\mathbb{J} \mathbb{I}^{\prime}[l]\right] \boldsymbol{\nu}^{\prime}$ where $\boldsymbol{\nu}^{\prime}=\boldsymbol{\nu}+$ $[1,1,0]^{T}$. Through algebraic manipulation we see that $\mathbf{c}_{l}^{\left(\mathbb{J}, \mathbb{I}^{\prime}\right)}+$ $\mathbf{P}\left[\mathbb{J} \mathbb{I}^{\prime}[l]\right] \boldsymbol{\nu}=\mathbf{v}_{1}=\mathbf{d}_{l-1}^{\mathbb{J}+}\left(\mathbb{I}^{\prime}\right)+\operatorname{sgn}\left(\mathbf{u}_{\mathbb{J}}^{T} \mathbf{p}_{\mathbb{I}[l]}\right) \mathbf{p}_{\mathbb{I}[l]}$ is equivalent to $\operatorname{sgn}\left(\mathbf{u}_{\mathbb{J}}^{T} \mathbf{p}_{\mathbb{I}[l]}\right) \mathbf{p}_{\mathbb{I}[l]}=\left(\chi^{-}\left(\mathbf{u}_{\mathbb{J}}^{T} \mathbf{p}_{\mathbb{I}^{\prime}[l]}\right)+\nu_{3}\right) \mathbf{p}_{\mathbb{I}^{\prime}[l]}+$ $\nu_{1} \mathbf{p}_{\mathbb{I}[1]}+\nu_{2} \mathbf{p}_{\mathbb{}[2]}$. From the linear independence of any set of three primaries, the preceding expression implies that both $\nu_{1}, \nu_{2} \neq 0$. Now if $\mathbf{v}_{1} \in \mathcal{P}_{l}^{\left(\mathbb{J}, \mathbb{I}^{\prime}\right)}$, then $1>\nu_{1}, \nu_{2}>0$ which implies $\nu_{1}^{\prime}, \nu_{2}^{\prime}>1$ and $\mathbf{v}_{2} \notin \mathcal{P}_{l}^{\left(\mathbb{J}, \mathbb{I}^{\prime}\right)}$. Conversely, if $\mathbf{v}_{2} \in \mathcal{P}_{l}^{\left(\mathbb{J}, \mathbb{I}^{\prime}\right)}$, then $0<\nu_{1}^{\prime}, \nu_{2}^{\prime}<1$ and $\nu_{1}, \nu_{2}<0$ and $\mathbf{v}_{1} \notin \mathcal{P}_{l}^{\left(J, \mathbb{I}^{\prime}\right)}$. Now choose $\mathbf{t} \in\left\{\mathbf{v}_{1}, \mathbf{v}_{2}\right\}$ such that $\mathbf{t} \notin \mathcal{P}_{l}^{\left(\mathbb{J}, \mathbb{I}^{\prime}\right)}$. Then $\mathbf{u}_{\mathbb{J}}^{T} \mathbf{d}_{l-1}^{\mathbb{J}+}(\mathbb{I})<\mathbf{u}_{\mathbb{J}}^{T} \mathbf{t}=\mathbf{u}_{\mathbb{J}}^{T} \mathbf{d}_{l}^{\mathbb{J}+}(\mathbb{I}) \leq \mathbf{u}_{\mathbb{J}}^{T} \mathbf{d}_{l}^{\mathbb{J}+}\left(\mathbb{I}^{\prime}\right)<$ $\mathbf{u}_{\sqrt{J}}^{T} \mathbf{d}_{l+1}^{\mathbb{J}+}\left(\mathbb{I}^{\prime}\right)<\mathbf{u}_{\mathfrak{J}}^{T} \mathbf{d}_{l+2}^{\mathbb{J}+}\left(\mathbb{I}^{\prime}\right)<\cdots<\mathbf{u}_{\mathbb{J}}^{T} \mathbf{d}_{K-2}^{\mathbb{J}+}\left(\mathbb{I}^{\prime}\right)$, where we have used the fact that the sequences $\left\{\mathbf{u}_{\mathbb{J}}^{T} \mathbf{d}_{m}^{\mathbb{I}+}(\mathbb{I})\right\}$ and $\left\{\mathbf{u}_{\mathbb{J}}^{T} \mathbf{d}_{m}^{\mathbb{I}+}\left(\mathbb{I}^{\prime}\right)\right\}$ are strictly increasing with $m$. Thus we can conclude that $\mathbf{t} \notin \mathcal{P}_{i}^{\left(J, I^{\prime}\right)}, \forall i \in\langle K-2\rangle$. It follows that $\mathbf{t} \notin \bigcup_{i=1}^{K-2} \mathcal{P}_{i}^{\left(\mathbb{J}, \mathbb{I}^{\prime}\right)}=\mathcal{Z}^{\left(\mathbb{J}, \mathbb{I}^{\prime}\right)}$, therefore, $\mathcal{Z}^{(\mathbb{J}, \mathbb{I})} \neq \mathcal{Z}^{\left(\mathbb{J}, \mathbb{I}^{\prime}\right)}$.

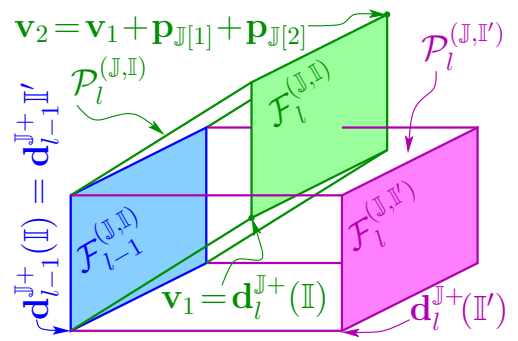

Fig. 7. For the facet spans $\mathcal{Z}^{(\mathbb{J}, \mathbb{I})}, \mathcal{Z}^{\left(\mathbb{J}, \mathbb{I}^{\prime}\right)}$ with $\mathbb{I} \neq \mathbb{I}^{\prime}$, the figure shows the first pair of parallelepipeds $\mathcal{P}_{l}^{(\mathbb{J}, \mathbb{I})}$ (green) and $\mathcal{P}_{l}^{\left(\mathbb{J}, \mathbb{I}^{\prime}\right)}$ (magenta), for which $\mathbb{I}[l] \neq \mathbb{I}^{\prime}[l]$. Note that $\mathcal{F}_{l-1}^{(\mathbb{J}, \mathbb{I})}=\mathcal{F}_{l-1}^{\left(\mathbb{J}, \mathbb{I}^{\prime}\right)}$ (blue) is a common facet for $\mathcal{P}_{l}^{(\mathbb{J}, \mathbb{I})}$ and $\mathcal{P}_{l}^{\left(\mathbb{J}, \mathbb{I}^{\prime}\right)}$ and of the two vertices $\mathbf{v}_{1}$ and $\mathbf{v}_{2}$ for the facet $\mathcal{F}_{l}^{(\mathbb{J}, \mathbb{I})}$, $\mathbf{v}_{1} \in \mathcal{P}_{l}^{\left(\mathbb{J}, \mathbb{I}^{\prime}\right)}$ and $\mathbf{v}_{2} \notin \mathcal{P}_{l}^{\left(\mathbb{J}, \mathbb{I}^{\prime}\right)}$, whereby $\mathbf{v}_{2} \notin \mathcal{Z}^{\left(\mathbb{J}, \mathbb{I}^{\prime}\right)}$.

\section{B. Proof of Lemma 2}

We start by establishing a relation between corresponding tristimuli in $\mathcal{Z}^{(\mathbb{J}, \mathbb{I})}$ and $\mathcal{Z}^{(\mathbb{J}, \tilde{I})}$; specifically, we show that, for any $l \in\langle K-2\rangle, \mathbf{t} \in \mathcal{P}_{l}^{(\mathbb{J}, \mathbb{I})}$, iff the tristimulus $\tilde{\mathbf{t}}=\mathbf{t}_{\mathbf{1}}-$ $\left(\mathbf{t}-\mathbf{t}_{\mathbf{0}}\right) \in \mathcal{P}_{K-1-l}^{(\mathbb{J}, \tilde{I})}$. Using the fact that $\tilde{\mathbb{I}}[K-1-l]=\mathbb{I}[l]$, we denote by $\mathbb{P}_{l}^{(\mathbb{I}, \mathbb{I})}=\mathbb{J} \cup \mathbb{I}[l]=\mathbb{J} \cup \tilde{\mathbb{I}}[K-1-l]$ the indices of the three primaries spanning both $\mathcal{P}_{l}^{(\mathbb{I}, \mathbb{I})}$ and $\mathcal{P}_{K-1-l}^{(\mathbb{J}, \tilde{\mathbb{I}})}$. Through algebraic manipulation, we can see that respective origins of $\mathcal{P}_{l}^{(\mathbb{I}, \mathbb{I})}$ and $\mathcal{P}_{K-1-l}^{(\mathbb{I}, \mathbb{I})}$ are

$$
\begin{aligned}
\mathbf{c}_{l}^{(\mathbb{I}, \mathbb{I})} & =\mathbf{t}_{\mathbf{0}}+\sum_{m=1}^{l-1} \chi^{+}\left(\mathbf{u}_{\mathbb{J}}^{T} \mathbf{p}_{\mathbb{I}[m]}\right) \mathbf{p}_{\mathbb{I}[m]}+\sum_{m=l+1}^{K-2} \chi^{-}\left(\mathbf{u}_{\mathbb{J}}^{T} \mathbf{p}_{\mathbb{I}[m]}\right) \mathbf{p}_{\mathbb{I}[m]}, \\
\mathbf{c}_{K-1-l}^{(\mathbb{I}, \tilde{I})} & =\mathbf{t}_{\mathbf{0}}+\sum_{m=1}^{l-1} \chi^{-}\left(\mathbf{u}_{\mathbb{J}}^{T} \mathbf{p}_{\mathbb{I}[m]}\right) \mathbf{p}_{\mathbb{I}[m]}+\sum_{m=l+1}^{K-2} \chi^{+}\left(\mathbf{u}_{\mathbb{J}}^{T} \mathbf{p}_{\mathbb{I}[m]}\right) \mathbf{p}_{\mathbb{I}[m]} .
\end{aligned}
$$

Noting that

$$
\begin{aligned}
\mathbf{t}_{\mathbf{1}}= & \mathbf{t}_{\mathbf{0}}+\sum_{k=1}^{K} \mathbf{p}_{k}=\mathbf{t}_{\mathbf{0}}+\sum_{\substack{m=1 \\
m \neq l}}^{K} \chi^{+}\left(\mathbf{u}_{\mathbb{J}}^{T} \mathbf{p}_{\mathbb{I}[m]}\right) \mathbf{p}_{\mathbb{I}[m]}+ \\
& \sum_{\substack{m=1 \\
m \neq l}}^{K} \chi^{-}\left(\mathbf{u}_{\mathbb{J}}^{T} \mathbf{p}_{\mathbb{I}[m]}\right) \mathbf{p}_{\mathbb{I}[m]}+\mathbf{P}\left[\mathbb{P}_{l}^{(\mathbb{I}, \mathbb{I})}\right] \mathbf{1},
\end{aligned}
$$

we can relate the origins of the parallelepipeds $\mathcal{P}_{l}^{(\mathbb{J}, \mathbb{I})}$ and $\mathcal{P}_{K-1-l}^{(J, \tilde{I})}$ as,

$$
\mathbf{c}_{K-1-l}^{(\mathbb{J}, \tilde{\mathbb{I}})}=\mathbf{t}_{\mathbf{1}}-\mathbf{c}_{l}^{(\mathbb{J}, \mathbb{I})}-\mathbf{P}\left[\mathbb{P}_{l}^{(\mathbb{J}, \mathbb{I})}\right] \mathbf{1}+\mathbf{t}_{\mathbf{0}} .
$$

Now, $\mathbf{t} \in \mathcal{P}_{l}^{(\mathbb{J}, \mathbb{I})}$ iff $\mathbf{t}=\mathbf{c}_{l}^{(\mathbb{J}, \mathbb{I})}+\mathbf{P}\left[\mathbb{P}_{l}^{(\mathbb{J}, \mathbb{I})}\right] \boldsymbol{\nu}$, with $\boldsymbol{\nu} \in[0,1]^{3}$, iff $\tilde{\mathbf{t}}=\mathbf{t}_{0}+\left(\mathbf{t}_{\mathbf{1}}-\mathbf{c}_{l}^{\left(\mathbb{J}, \mathbb{I}_{\mathrm{J}}\right)}-\mathbf{P}\left[\mathbb{P}_{l}^{\left(\mathbb{J}, \mathbb{I}_{\mathrm{I}}\right)}\right] \boldsymbol{\nu}\right)=$ $\mathbf{t}_{0}+\left(\mathbf{t}_{\mathbf{1}}-\mathbf{c}_{l}^{\left(\mathbb{J}, \mathbb{I}_{\mathrm{J}}\right)}-\mathbf{P}\left[\mathbb{P}_{l}^{\left(\mathbb{J}, \mathbb{I}_{\mathrm{J}}\right)}\right](\mathbf{1}-\tilde{\boldsymbol{\nu}})\right)$, and from (15), iff 
$\tilde{\mathbf{t}}=\mathbf{c}_{K-1-l}^{(\mathbb{J}, \tilde{\mathbb{I}})}+\mathbf{P}\left[\mathbb{P}_{l}^{(\mathbb{J}, \mathbb{I})}\right] \tilde{\boldsymbol{\nu}}$, iff $\tilde{\mathbf{t}} \in \mathcal{P}_{K-1-l}^{(\mathbb{J}, \tilde{\mathbb{I}})}$, where $\tilde{\boldsymbol{\nu}}=$ $\mathbf{1}-\boldsymbol{\nu} \in[0,1]^{3}$. Moreover, $\mathbf{t}$ is in a proper face of $\mathcal{P}_{l}^{(\mathbb{J}, \mathbb{I})}$ iff $\boldsymbol{\nu}$ has at least one vector component in $\{0,1\}$, iff $\tilde{\boldsymbol{\nu}}$ has at least one vector component in $\{0,1\}$, iff $\tilde{\mathbf{t}}$ is in a proper face of $\mathcal{P}_{K-1-l}^{(\mathbb{I}, \tilde{I})}$. Analogous statements follow for the relationship between the tristimuli of the parallelepipeds $\mathcal{P}_{l}^{\left(\mathbb{J}^{\prime}, \mathbb{I}^{\prime}\right)}$ and $\mathcal{P}_{K-1-l}^{\left(\mathbb{J}^{\prime}, \tilde{\mathbb{I}}^{\prime}\right)}$ of the facet spans $\mathcal{Z}^{\left(\mathbb{J}^{\prime}, \mathbb{I}^{\prime}\right)}$ and $\mathcal{Z}^{\left(\mathbb{J}^{\prime}, \tilde{\mathbb{I}}^{\prime}\right)}$. Therefore, for every $l, l^{\prime} \in\langle K-2\rangle, \mathcal{P}_{l}^{(\mathbb{J}, \mathbb{I})} \cap \mathcal{P}_{l^{\prime}}^{\left(\mathbb{J}^{\prime}, \mathbb{I}^{\prime}\right)}$ is a common $d$-face of $\mathcal{P}_{l}^{(\mathbb{J}, \mathbb{I})}$ and $\mathcal{P}_{l^{\prime}}^{\left(\mathbb{J}^{\prime}, \mathbb{I}^{\prime}\right)}$ iff $\mathcal{P}_{K-1-l}^{(\mathbb{J}, \tilde{\mathbb{I}})} \cap \mathcal{P}_{K-1-l^{\prime}}^{\left(\mathbb{J}^{\prime}, \tilde{\mathbb{I}}^{\prime}\right)}$ is a common $d$ face of $\mathcal{P}_{K-1-l}^{(\mathbb{J}, \tilde{\mathbb{I}})}$ and $\mathcal{P}_{K-1-l^{\prime}}^{\left(\mathbb{J}^{\prime}, \tilde{I}^{\prime}\right)}$, where $0 \leq d \leq 3$ denotes the dimensionality of the face. It follows that $\mathcal{Z}^{(\mathbb{J}, \mathbb{I})}$ and $\mathcal{Z}^{\left(\mathbb{J}^{\prime}, \mathbb{I}^{\prime}\right)}$ are compatible iff $\mathcal{Z}^{(\mathbb{J}, \tilde{\mathbb{I}})}$ and $\mathcal{Z}^{\left(\mathbb{J}^{\prime}, \tilde{\mathbb{I}}^{\prime}\right)}$ are compatible.

\section{Proof of Lemma 3}

Let $\mathcal{K}$ be a maximal set. We shall establish that $\mathcal{T}_{\mathcal{K}}$ is a gamut tiling by showing that the set $\mathcal{A}=\bigcup_{\mathcal{P} \in \mathcal{T}_{\mathcal{K}}} \mathcal{P}$ is the gamut $\mathcal{G}$. Because every $\mathcal{P} \in \mathcal{T}_{\mathcal{K}}$ is a subset of a facet span, we see that $\mathcal{P} \subseteq \mathcal{G}$, therefore, $\mathcal{A} \subseteq \mathcal{G}$. Now, let $\mathcal{T}^{\prime}$ be a progressive tiling of the gamut. Observing that both $\mathcal{T}_{\mathcal{K}}$ and $\mathcal{T}^{\prime}$, are collections of essentially disjoint parallelepipeds having exactly one parallelepiped spanned by primaries indexed by every $\mathbb{P} \in \mathfrak{C}^{3}(\langle K\rangle)$, we see that every $\mathcal{P} \in \mathcal{T}_{\mathcal{K}}$ is a displacement of some $\mathcal{P}^{\prime} \in \mathcal{T}^{\prime}$. Furthermore, because $\mathcal{T}_{\mathcal{K}}$ and $\mathcal{T}^{\prime}$ are collections of essentially disjoint parallelepipeds, we have $\mathscr{V}(\mathcal{A})=\sum_{\mathcal{P} \in \mathcal{T}_{\mathcal{K}}} \mathscr{V}(\mathcal{P})=\sum_{\mathcal{P}^{\prime} \in \mathcal{T}_{\mathcal{K}}^{\prime}} \mathscr{V}\left(\mathcal{P}^{\prime}\right)=\mathscr{V}(\mathcal{G})$. Now $\mathcal{A}$ and $\mathcal{G}$ are both closed sets with identical volume with $\mathcal{A} \subseteq \mathcal{G}$ and $\mathcal{G}$ is convex, whereby we can conclude that $\mathcal{A}=\mathcal{G}$, therefore, $\mathcal{T}_{\mathcal{K}}$ is a gamut tiling.

\section{Proof of Lemma 4}

We start by showing the one-to-one correspondence between the complete sets and the gamut tilings. For $K=3$ the result is direct, so assume $K \geq 4$. Because for a complete set $\mathcal{K}$, the collection $\mathcal{T}_{\mathcal{K}}$ of all parallelepipeds making up the facet spans of $\mathcal{K}$ is a gamut tiling, the mapping $h: \mathcal{K} \mapsto \mathcal{T}_{\mathcal{K}}$ is a function from complete sets to gamut tilings. We first prove that $h$ is one-to-one. Let $\mathcal{K}$ and $\mathcal{K}^{\prime}$ be two different complete sets. Because $\mathcal{K}$ and $\mathcal{K}^{\prime}$ are sets with a facet span for every pair of primaries, there is $\mathbb{J} \in \mathfrak{C}^{2}(\langle K\rangle)$ for which the corresponding facet spans $\mathcal{Z}^{(\mathbb{J}, \mathbb{I})} \in \mathcal{K}$ and $\mathcal{Z}^{\left(\mathbb{J}, \mathbb{I}^{\prime}\right)} \in \mathcal{K}^{\prime}$ are different, where $\mathbb{I}, \mathbb{I}^{\prime} \in \mathfrak{P}(\langle K\rangle \backslash \mathbb{J})$. And because $\mathcal{Z}^{(\mathbb{J}, \mathbb{I})} \neq \mathcal{Z}^{\left(\mathbb{J}, \mathbb{I}^{\prime}\right)}$, we conclude from Lemma 1 that $\mathbb{I} \neq \mathbb{I}^{\prime}$. The situation is illustrated in Fig. 8. Let $l$ be the smallest integer such that $\mathbb{I}[l] \neq \mathbb{I}^{\prime}[l]$. We see from (7) that $\mathbf{d}_{l-1}^{\mathbb{J}+}(\mathbb{I})=\mathbf{d}_{l-1}^{\mathbb{J}+}\left(\mathbb{I}^{\prime}\right)$ indicating that $\mathcal{F}_{l-1}^{(\mathbb{J}, \mathbb{I})}=\mathcal{F}_{l-1}^{\left(\mathbb{J}, \mathbb{I}^{\prime}\right)}$ is a common facet for $\mathcal{P}_{l}^{(\mathbb{J}, \mathbb{I})}$ and $\mathcal{P}_{l}^{\left(\mathbb{J}, \mathbb{I}^{\prime}\right)}$. Assume, without loss of generality, that $\mathbf{u}_{\mathbb{J}}^{T} \mathbf{d}_{l}^{\mathbb{J}+}(\mathbb{I}) \leq \mathbf{u}_{\mathbb{J}}^{T} \mathbf{d}_{l}^{\mathbb{J}+}\left(\mathbb{I}^{\prime}\right)$ (otherwise simply swap $\mathbb{I}$ and $\mathbb{I}^{\prime}$ ), and let $l<l^{\prime} \leq(K-2)$ be the index such that $\mathbb{I}^{\prime}\left[l^{\prime}\right]=\mathbb{I}[l]$. Note that $l^{\prime}$ is well defined because $\mathbb{I}, \mathbb{I}^{\prime} \in \mathfrak{P}(\langle K\rangle \backslash \mathbb{J})$. Then, the parallelepipeds $\mathcal{P}_{l}^{(\mathbb{J}, \mathbb{I})}$ and $\mathcal{P}_{l^{\prime}}^{\left(\mathbb{J}, \mathbb{I}^{\prime}\right)}$ are both spanned by the primaries indexed by $\mathbb{P}=\mathbb{J} \cup \mathbb{I}[l]=\mathbb{J} \cup \mathbb{I}^{\prime}\left[l^{\prime}\right]$. Using $\mathbb{I}[l]=\mathbb{I}^{\prime}\left[l^{\prime}\right]$, we see that the difference between the origins of $\mathcal{P}_{l}^{(\mathbb{J}, \mathbb{I})}$ and $\mathcal{P}_{l^{\prime}}^{\left(\mathbb{J}, \mathbb{I}^{\prime}\right)}$ is the vector $\mathbf{c}_{l^{\prime}}^{\left(\mathbb{J}, \mathbb{I}^{\prime}\right)}-\mathbf{c}_{l}^{(\mathbb{J}, \mathbb{I})}=\mathbf{d}_{l^{\prime}}^{\mathbb{J}+}\left(\mathbb{I}^{\prime}\right)-\mathbf{d}_{l}^{\mathbb{J}+}(\mathbb{I})$. Because $l<l^{\prime}$, we have $\mathbf{u}_{\mathbb{J}}^{T} \mathbf{d}_{l}^{\mathbb{J}+}(\mathbb{I}) \leq \mathbf{u}_{\mathbb{J}}^{T} \mathbf{d}_{l}^{\mathbb{J}+}\left(\mathbb{I}^{\prime}\right)<\mathbf{u}_{\mathbb{J}}^{T} \mathbf{d}_{l^{\prime}}^{\mathbb{J}+}\left(\mathbb{I}^{\prime}\right)$, where we have used the fact that the sequence $\left\{\mathbf{u}_{\mathbb{J}}^{T} \mathbf{d}_{m}^{\mathbb{J}+}\left(\mathbb{I}^{\prime}\right)\right\}$ is strictly increasing with $m$. It follows that $\mathbf{u}_{\mathbb{J}}^{T}\left(\mathbf{d}_{l^{\prime}}^{\mathbb{J}+}\left(\mathbb{I}^{\prime}\right)-\mathbf{d}_{l}^{\mathbb{J}+}(\mathbb{I})\right) \neq 0$, thus, $\mathbf{d}_{l^{\prime}}^{\mathbb{J}+}\left(\mathbb{I}^{\prime}\right)-\mathbf{d}_{l}^{\mathbb{J}+}(\mathbb{I}) \neq \mathbf{0}$, so $\mathbf{c}_{l^{\prime}}^{\left(\mathbb{J}, \mathbb{I}^{\prime}\right)} \neq \mathbf{c}_{l}^{(\mathbb{J}, \mathbb{I})}$, therefore, $\mathcal{P}_{l^{\prime}}^{\left(\mathbb{J}, \mathbb{I}^{\prime}\right)} \neq \mathcal{P}_{l}^{(\mathbb{J}, \mathbb{I})}$. Because $\mathcal{P}_{l}^{(\mathbb{J}, \mathbb{I})}$ and $\mathcal{P}_{l^{\prime}}^{\left(\mathbb{J}, \mathbb{I}^{\prime}\right)}$ are the only parallelepipeds that are spanned by $\mathbb{P}$ in the collections $\mathcal{T}_{\mathcal{K}}$ and $\mathcal{T}_{\mathcal{K}^{\prime}}$, respectively, we see that $\mathcal{P}_{l}^{(\mathbb{J}, \mathbb{I})} \notin \mathcal{T}_{\mathcal{K}^{\prime}}, \mathcal{P}_{l^{\prime}}^{\left(\mathbb{J}, \mathbb{I}^{\prime}\right)} \notin \mathcal{T}_{\mathcal{K}}$, therefore, $\mathcal{T}_{\mathcal{K}} \neq \mathcal{T}_{\mathcal{K}^{\prime}}$, so $h$ maps different complete sets to different gamut tilings, establishing that $h$ is one-to-one.
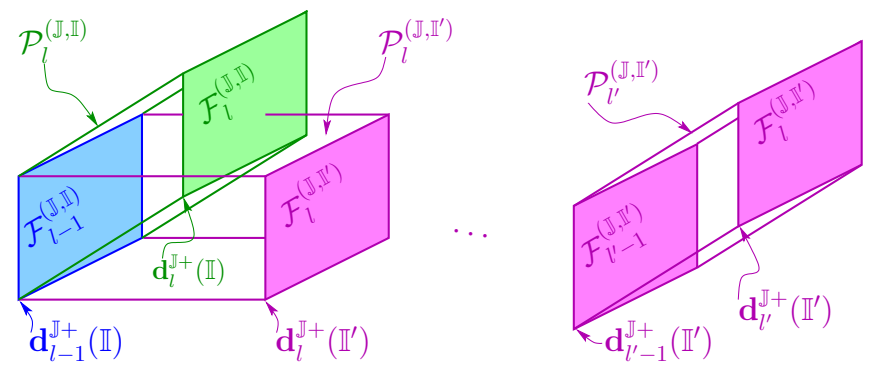

Fig. 8. For the facet spans $\mathcal{Z}^{(\mathbb{J}, \mathbb{I})}, \mathcal{Z}^{\left(\mathbb{J}, \mathbb{I}^{\prime}\right)}$ with $\mathbb{I} \neq \mathbb{I}^{\prime}$, the figure shows the first pair of parallelepipeds $\mathcal{P}_{l}^{(\mathbb{J}, \mathbb{I})}$ (green) and $\mathcal{P}_{l}^{\left(\mathbb{J}, \mathbb{I}^{\prime}\right)}$ (magenta), for which $\mathbb{I}[l] \neq \mathbb{I}^{\prime}[l]$, which share the facet $\mathcal{F}_{l-1}^{(\mathbb{J}, \mathbb{I})}=\mathcal{F}_{l-1}^{\left(\mathbb{J}, \mathbb{I}^{\prime}\right)}$ (blue). The parallelepiped $\mathcal{P}_{l^{\prime}}^{\left(\mathbb{I}, \mathbb{I}^{\prime}\right)}$ (magenta), which is spanned by the same primaries as $\mathcal{P}_{l}^{(\mathbb{J}, \mathbb{I})}$ is also shown. It can be seen that (see text) $\mathcal{P}_{l}^{(\mathbb{I}, \mathbb{I})}$ and $\mathcal{P}_{l^{\prime}}^{\left(\mathbb{I}, \mathbb{I}^{\prime}\right)}$ cannot be part of the same tiling.

Next we show that $h$ is onto. Let $\mathcal{T}$ be a gamut tiling. We construct a complete set $\mathcal{K}$ such that $\mathcal{T}_{\mathcal{K}}=\mathcal{T}$ as follows. Let $\mathbb{J} \in \mathfrak{C}^{2}(\langle K\rangle)$, let $\mathcal{T}_{\mathbb{J}}$ be the subset of parallelepipeds from $\mathcal{T}$ with facets spanned by the pair of primaries $\mathbf{P}[\mathbb{J}]$. Because $\mathcal{T}$ is a gamut tiling, it includes one parallelepiped for each $\mathbb{P} \in \mathfrak{C}^{3}(\langle K\rangle)$, and we see that $\mathcal{I}_{\mathbb{J}}$ has $(K-2)$ parallelepipeds, one per index triple $\mathbb{J} \cup i$, for all $i \in\langle K\rangle \backslash \mathbb{J}$. Next, consider the following recursive procedure for arranging the parallelepipeds in $\mathcal{T}_{\mathbb{J}}$ in a sequence $\mathcal{P}_{1}, \ldots, \mathcal{P}_{K-2}$ and for creating an associated sequence of indices $\mathbb{I} \in \mathfrak{P}(\langle K\rangle \backslash \mathbb{J})$. Let $\mathcal{F}_{0}=\mathcal{F}\left(\mathbf{d}^{\mathbb{J}-}, \mathbf{P}[\mathbb{J}]\right)$ and let $\mathbf{v}_{0}=\mathbf{d}^{\mathbb{J}-}$ denote the origin of $\mathcal{F}_{0}$. Because $\mathcal{T}$ is a gamut tiling, it follows that there is a parallelepiped $\mathcal{P}_{1} \in \mathcal{T}$ for which $\mathcal{F}_{0}$ is one of its facets, whereby $\mathcal{P}_{1} \in \mathcal{T}_{\mathbb{J}}$. Let $\mathcal{F}_{1}$ be the companion facet of $\mathcal{F}_{0}$ in $\mathcal{P}_{1}$, i.e., the facet that is also spanned by $\mathbf{P}[\mathbb{J}]$ and congruent with $\mathcal{F}_{0}$. Now denote by $\mathbb{P}_{1}$ the index triple for the primaries spanning $\mathcal{P}_{1}$, and set $\mathbb{I}[1]=\mathbb{P}_{1} \backslash \mathbb{J}$ (note $\mathbb{I}[l] \in\langle K\rangle \backslash \mathbb{J}$ ). Then $\mathbf{v}_{1}=\mathbf{v}_{0}+\operatorname{sgn}\left(\mathbf{u}_{\mathbb{J}}^{T} \mathbf{p}_{\mathbb{I}[1]}\right) \mathbf{p}_{\mathbb{I}[1]}$ is the origin of $\mathcal{F}_{1}$ and $\mathbf{u}_{\mathbb{J}}^{T} \mathbf{d}^{\mathbb{J}-}=\mathbf{u}_{\mathbb{J}}^{T} \mathbf{v}_{0}<\mathbf{u}_{\mathbb{J}}^{T} \mathbf{v}_{1}<\mathbf{u}_{\mathbb{J}}^{T} \mathbf{d}^{\mathbb{J}+}$. For $2 \leq l \leq K-2$ select $\mathcal{P}_{l} \in \mathcal{T}_{\mathbb{J}} \backslash\left\{\mathcal{P}_{1}, \ldots, \mathcal{P}_{l-1}\right\}$ and set $\mathbb{I}[l]$ as follows. Let $\mathbf{t} \in \operatorname{Int}\left(\mathcal{F}_{l-1}\right)$. Because $\mathbf{u}_{\mathfrak{J}}^{T} \mathbf{d}^{\mathbb{J}-}<\mathbf{u}_{\mathbb{J}}^{T} \mathbf{v}_{l-1}<\mathbf{u}_{\mathfrak{J}}^{T} \mathbf{d}^{\mathbb{J}+}$, we see that $\mathcal{F}_{l-1}$ is not a gamut facet, so $\mathbf{t} \in \operatorname{Int}(\mathcal{G})$. Observing that $\mathbf{u}_{\mathfrak{J}}^{T} \mathbf{v}_{0}<\mathbf{u}_{\mathbb{J}}^{T} \mathbf{v}_{1}<\cdots<\mathbf{u}_{\mathfrak{J}}^{T} \mathbf{v}_{l-1}=\mathbf{u}_{\mathfrak{J}}^{T} \mathbf{t}$ and because $\mathcal{T}$ is a gamut tiling, there exists $\mathcal{P}_{l} \in \mathcal{T} \backslash\left\{\mathcal{P}_{1}, \ldots, \mathcal{P}_{l-1}\right\}$ such that $\mathbf{t} \in \mathcal{P}_{l} \cap \mathcal{P}_{l-1}$, where $\mathcal{P}_{l} \cap \mathcal{P}_{l-1}$ is a face of $\mathcal{P}_{l}$ and $\mathcal{P}_{l-1}$. Because $\mathcal{F}_{l-1}$ is the only face of $\mathcal{P}_{l-1}$ containing $\mathbf{t}$, we see that $\mathcal{P}_{l} \cap \mathcal{P}_{l-1}=\mathcal{F}_{l-1}$, so $\mathcal{F}_{l-1}$ is also a facet of $\mathcal{P}_{l}$, therefore, $\mathcal{P}_{l} \in \mathcal{T}_{\mathbb{J}}$. Let $\mathbb{P}_{l}$ the index 
triple for the primaries spanning $\mathcal{P}_{l}$, and set $\mathbb{I}[l]=\mathbb{P}_{l} \backslash \mathbb{J}$ (note $\mathbb{I}[l] \in\langle K\rangle \backslash \mathbb{J}$ is distinct from $\mathbb{I}[0], \mathbb{I}[1], \ldots \mathbb{I}[l-1]$ ). Let $\mathcal{F}_{l}$ be the companion facet of $\mathcal{F}_{l-1}$ in $\mathcal{P}_{l}$ and then $\mathbf{v}_{l}=\mathbf{v}_{l-1}+\operatorname{sgn}\left(\mathbf{u}_{\mathbb{J}}^{T} \mathbf{p}_{\mathbb{I}[l]}\right) \mathbf{p}_{\mathbb{I}[l]}$ is the origin of $\mathcal{F}_{l}$, and for $l<(K-2), \mathbf{u}_{\mathbb{J}}^{T} \mathbf{v}_{l-1}<\mathbf{u}_{\mathbb{J}}^{T} \mathbf{v}_{l}<\mathbf{u}_{\mathbb{J}}^{T} \mathbf{d}^{\mathbb{J}+}$, which establishes that the recursive procedure can continue until $l=(K-2)$ at which point $\mathbf{v}_{K-2}=\mathbf{v}_{0}+\sum_{j=1}^{K-2} \operatorname{sgn}\left(\mathbf{u}_{\mathbb{J}}^{T} \mathbf{p}_{\mathbb{I}[j]}\right) \mathbf{p}_{\mathbb{I}[j]}=$ $\mathbf{d}^{\mathbb{J}-}+\sum_{j \in\langle K\rangle \backslash \mathbb{J}} \operatorname{sgn}\left(\mathbf{u}_{\mathbb{J}}^{T} \mathbf{p}_{j}\right) \mathbf{p}_{j}=\mathbf{d}^{\mathbb{J}+}$, and $\mathcal{F}_{K-2}=$ $\mathcal{F}\left(\mathbf{d}^{\mathbb{J}+}, \mathbf{P}[\mathbb{J}]\right)$. Because the process selects $(K-2)$ distinct parallelepipeds from the set $\mathcal{T}_{\mathbb{J}}$ with $(K-2)$ distinct elements, the choice at each stage of the recursion is unique. It follows that $\mathbb{I} \in \mathfrak{P}(\langle K\rangle \backslash \mathbb{J})$, and the set $\bigcup_{\mathcal{P} \in \mathcal{T}_{\mathbb{J}}} \mathcal{P}=\bigcup_{l=1}^{K-2} \mathcal{P}_{l}$ constitutes the facet span $\mathcal{Z}^{\left(\mathbb{J}, \mathbb{I}_{T}\right)}$, where $\mathbb{I}_{\mathcal{T}}=\mathbb{I}$ with the subscript added to indicate the dependence in subsequent discussion.

Repeating the preceding process for each $\mathbb{J} \in \mathfrak{C}^{2}(\langle K\rangle)$, we obtain the set of facet spans $\mathcal{K}=\left\{\mathcal{Z}^{\left(\mathbb{J}, \mathbb{I}_{\mathcal{T}}\right)}=\bigcup_{\mathcal{P} \in \mathcal{T}_{\mathbb{T}}} \mathcal{P} \mid \mathbb{J} \in\right.$ $\left.\mathfrak{C}^{2}(\langle K\rangle)\right\}$. Because $\mathcal{T}$ is a gamut tiling, the facet spans in $\mathcal{K}$ are compatible, making $\mathcal{K}$ a complete set, with $\mathcal{T}_{\mathcal{K}}=\mathcal{T}$. Therefore, $h$ is onto, and a bijection between complete sets and gamut tilings.

We now show that every maximal set is a subset of one and only one complete set. Let $\mathcal{K}$ be a maximal set with $\mathcal{T}_{\mathcal{K}}$ denoting the associated tiling as per our notational convention. Now from our preceding result, $\mathcal{K}^{\prime}=h^{-1}\left(\mathcal{T}_{\mathcal{K}}\right)$ is the unique complete set associated with the tiling $\mathcal{T}_{\mathcal{K}}$ and $\mathcal{T}_{\mathcal{K}^{\prime}}=h\left(\mathcal{K}^{\prime}\right)=\mathcal{T}_{\mathcal{K}}$. Now, via the recursive construction procedure used in the preceding part, the parallelepipeds in the gamut tiling $\mathcal{T}_{\mathcal{K}}=\mathcal{T}_{\mathcal{K}^{\prime}}$ yield a unique facet span for each $\mathbb{J} \in \mathfrak{C}^{2}(\langle K\rangle)$, it follows that every facet span in $\mathcal{K}$ is a facet span of $\mathcal{K}^{\prime}$ and therefore, $\mathcal{K} \subseteq \mathcal{K}^{\prime}$. And because $h$ is one-toone, $\mathcal{K}^{\prime}$ is the only complete set such that $\mathcal{T}_{\mathcal{K}^{\prime}}=\mathcal{T}_{\mathcal{K}}$, therefore, the only complete set containing $\mathcal{K}$.

We now establish that every facet span belongs to a complete set using induction on the number of primaries $K$. The result follows immediately for $K=3$. Next, let $M \geq 4$. We assume that the result holds for all systems with $K \leq(M-1)$ primaries and show that it holds for $K=M$. For the $M$ primary system, denote by $\mathbf{P}$ the $3 \times M$ primary matrix, $\mathbf{t}_{\mathbf{0}} \in \mathbb{R}^{3}$ the display black, and $\mathcal{G}$ the gamut. Let $\mathcal{Z}^{\left(\mathbb{J}_{0}, \mathbb{I}_{0}\right)}$ be a facet span, with $\mathbb{J}_{0} \in \mathfrak{C}^{2}(\langle M\rangle)$ and $\mathbb{I}_{0} \in \mathfrak{P}\left(\langle M\rangle \backslash \mathbb{J}_{0}\right)$, and assume without loss of generality, that $M$ is the last index of $\mathbb{I}_{0}$, that is, $\mathbb{I}_{0}[M-2]=M$ (otherwise, permute the columns in $\mathbf{P}$ accordingly). We will show that $\mathcal{Z}^{\left(\mathbb{J}_{0}, \mathbb{I}_{0}\right)}$ belongs to a complete set. Let $\check{\mathbf{P}}=\mathbf{P}[\langle M-1\rangle]$ and $\check{\mathbf{t}}_{\mathbf{0}}=\mathbf{t}_{\mathbf{0}}+\chi^{-}\left(\mathbf{u}_{\mathbb{J}_{0}}^{T} \mathbf{p}_{M}\right) \mathbf{p}_{M}$, which together define a $(M-1)$-primary display system with gamut $\check{\mathcal{G}}=\left\{\check{\mathbf{P}} \check{\boldsymbol{\alpha}}+\check{\mathbf{t}}_{\mathbf{0}} \mid \check{\boldsymbol{\alpha}} \in[0,1]^{(M-1)}\right\}$. To simplify the ensuing discussion, we extend the convention implicit in our preceding definitions and denote by $\check{x}$ and $x$ corresponding variables associated with the $(M-1)$ and $M$ primary systems, respectively. Now, let $\check{\mathbb{I}}_{0}=\mathbb{I}_{0}[1,2, \ldots,(M-3)]$. Then observing that $\mathbf{d}^{\mathbb{J}_{0}-}=\mathbf{t}_{\mathbf{0}}+\mathbf{P} \chi^{\mathbb{J}_{0}-}=\mathbf{t}_{\mathbf{0}}+\chi^{-}\left(\mathbf{u}_{\mathbb{J}_{0}}^{T} \mathbf{p}_{M}\right) \mathbf{p}_{M}+\check{\mathbf{P}} \check{\boldsymbol{\chi}}^{\mathbb{J}_{0}-}=$ $\check{\mathbf{t}}_{\mathbf{0}}+\check{\mathbf{P}} \check{\chi}^{\mathbb{J}_{0}-}=\check{\mathbf{d}}^{\mathbb{J}_{0}-}$, we see that $\check{\mathcal{Z}}^{\left(\mathbb{J}_{0}, \check{\mathbb{I}}_{0}\right)}=\bigcup_{l=1}^{M-3} \check{\mathcal{P}}_{l}^{\left(\mathbb{J}_{0}, \check{\mathbb{I}}_{0}\right)}$ is a facet span for the $(M-1)$ primary system, where $\check{\mathcal{P}}_{l}^{\left(\mathbb{J}_{0}, \check{\mathbb{I}}_{0}\right)}=\mathcal{P}_{l}^{\left(\mathbb{J}_{0}, \mathbb{I}_{0}\right)}, l=1,2, \ldots(M-3)$.

Then, via the induction hypothesis, there is a complete set $\check{\mathcal{K}}$ of facet spans in $\breve{\mathcal{G}}$ with associated gamut tiling $\mathcal{T}_{\check{\mathcal{K}}}$, such that $\check{\mathcal{Z}}^{\left(\mathbb{J}_{0}, \breve{I}_{0}\right)} \in \check{\mathcal{K}}$. We extend the facet spans in $\check{\mathcal{K}}$ to obtain a set of compatible facet spans for the $M$-primary system. The process is illustrated in Fig. 9. Specifically, for $\mathbb{J} \in \mathfrak{C}^{2}(\langle M-1\rangle)$ if $\chi^{-}\left(\mathbf{u}_{\mathbb{J}}^{T} \mathbf{p}_{M}\right)=\chi^{-}\left(\mathbf{u}_{\mathbb{J}_{0}}^{T} \mathbf{p}_{M}\right)$, define $\mathbb{I}=[\check{I}, M]$, otherwise define $\mathbb{I}=[M, \check{I}]$. In both cases, it can be seen that the collection of facet spans $\mathcal{Z}^{(\mathbb{J}, \mathbb{I})}, \mathbb{J} \in \mathfrak{C}^{2}(\langle M-1\rangle)$ is a set of compatible facet spans for $\mathcal{G}_{M}$ with $\mathcal{Z}^{\left(\mathbb{J}_{0}, \mathbb{I}\right)}=$ $\mathcal{Z}^{\left(\mathbb{J}_{0}, \mathbb{I}_{0}\right)}$. Specifically, if $\chi^{-}\left(\mathbf{u}_{\sqrt{J}}^{T} \mathbf{p}_{M}\right)=\chi^{-}\left(\mathbf{u}_{\mathbb{J}_{0}}^{T} \mathbf{p}_{M}\right)$, we have $\check{\mathbf{d}}^{\mathbb{J}-}=\check{\mathbf{t}}_{\mathbf{0}}+\check{\mathbf{P}} \check{\chi}^{\mathbb{J}-}=\mathbf{t}_{\mathbf{0}}+\chi^{-}\left(\mathbf{u}_{\mathbb{J}_{0}}^{T} \mathbf{p}_{M}\right) \mathbf{p}_{M}+\check{\mathbf{P}} \check{\chi}^{\mathbb{J}-}=$ $\mathbf{t}_{\mathbf{0}}+\chi^{-}\left(\mathbf{u}_{\mathbb{J}}^{T} \mathbf{p}_{M}\right) \mathbf{p}_{M}+\check{\mathbf{P}} \check{\chi}^{\mathbb{J}-}=\mathbf{t}_{\mathbf{0}}+\mathbf{P} \chi^{\mathbb{J}-}=\mathbf{d}^{\mathbb{J}-}$, and we see that $\check{\mathbf{d}}_{l}^{\mathbb{J}+}(\check{\mathbb{I}})=\mathbf{d}_{l}^{\mathbb{I}+}(\mathbb{I})$ and $\check{\mathcal{P}}_{l}^{(\mathbb{I}, \check{\mathbb{I}})}=\mathcal{P}_{l}^{(\mathbb{I}, \mathbb{I})}$, for $l \in\langle M-3\rangle$, and $\mathcal{Z}^{(\mathbb{J}, \mathbb{I})}=\check{\mathcal{Z}}^{(\mathbb{J}, \mathbb{I})} \cup \mathcal{P}_{M-2}^{(\mathbb{J}, \mathbb{I})}$. On the other hand, if $\chi^{-}\left(\mathbf{u}_{\sqrt{J}}^{T} \mathbf{p}_{M}\right) \neq \chi^{-}\left(\mathbf{u}_{\mathbb{J}_{0}}^{T} \mathbf{p}_{M}\right)$, we have $\chi^{-}\left(\mathbf{u}_{\mathbb{J}_{0}}^{T} \mathbf{p}_{M}\right)=\chi^{+}\left(\mathbf{u}_{\mathbb{J}}^{T} \mathbf{p}_{M}\right)$, thus, $\check{\mathbf{d}}^{\mathbb{J}-}=\check{\mathbf{t}}_{\mathbf{0}}+\check{\mathbf{P}} \check{\chi}^{\mathbb{J}-}=$ $\mathbf{t}_{\mathbf{0}}+\chi^{-}\left(\mathbf{u}_{\mathbb{J}_{0}}^{T} \mathbf{p}_{M}\right) \mathbf{p}_{M}+\check{\mathbf{P}} \check{\chi}^{\mathbb{J}-}=\mathbf{t}_{\mathbf{0}}+\chi^{+}\left(\mathbf{u}_{\mathbb{J}}^{T} \mathbf{p}_{M}\right) \mathbf{p}_{M}+$ $\check{\mathbf{P}} \check{\chi}^{\mathbb{J}-}=\mathbf{t}_{\mathbf{0}}+\mathbf{P} \boldsymbol{\chi}^{\mathbb{J}-}+\left(\chi^{+}\left(\mathbf{u}_{\mathbb{J}}^{T} \mathbf{p}_{M}\right)-\chi^{-}\left(\mathbf{u}_{\sqrt{J}}^{T} \mathbf{p}_{M}\right)\right) \mathbf{p}_{M}=$ $\mathbf{d}^{\mathbb{J}-}+\operatorname{sgn}\left(\mathbf{u}_{\mathbb{J}}^{T} \mathbf{p}_{M}\right) \mathbf{p}_{M}=\mathbf{d}^{\mathbb{J}-}+\operatorname{sgn}\left(\mathbf{u}_{\mathbb{J}}^{T} \mathbf{p}_{\mathbb{I}[1]}\right) \mathbf{p}_{\mathbb{I}[1]}$. It follows that $\check{\mathbf{d}}_{l}^{\mathbb{I}+}(\check{\mathbb{I}})=\mathbf{d}_{l+1}^{\mathbb{J}+}(\mathbb{I})$ and $\check{\mathcal{P}}_{l}^{(\mathbb{J}, \check{\mathbb{I}})}=\mathcal{P}_{l+1}^{(\mathbb{J}, \mathbb{I})}$, for $l \in\langle M-3\rangle$, and $\mathcal{Z}^{(\mathbb{J}, \mathbb{I})}=\mathcal{P}_{1}^{(\mathbb{I}, \mathbb{I})} \cup \check{\mathcal{Z}}^{(\mathbb{J}, \check{I})}$.

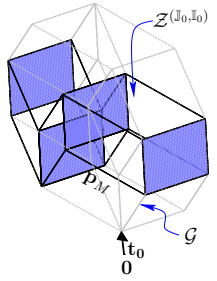

(a)

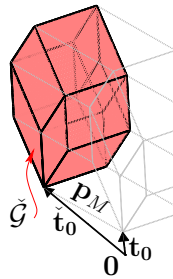

(b)

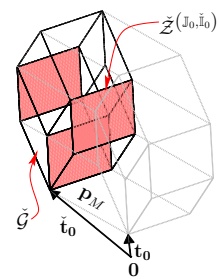

(c)

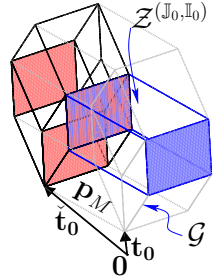

(d)
Fig. 9. Induction step for illustrating that every facet span belongs to a complete set. See description in text.

To establish that the extensions are compatible facet spans, for all $\mathbb{J} \in \mathfrak{C}^{2}(\langle M-1\rangle)$, we denote by $\mathcal{P}_{\mathbb{J}} \stackrel{\text { def }}{=} \mathcal{Z}^{(\mathbb{J}, \mathbb{I})} \backslash \check{\mathcal{Z}}^{(\mathbb{J}, \breve{I})}$ the parallelepiped added in the extension $\mathcal{Z}^{(\mathbb{J}, \mathbb{I})}$. Let $\mathbb{J}, \mathbb{J}^{\prime} \in$ $\mathfrak{C}^{2}(\langle M-1\rangle), \mathbb{J} \neq \mathbb{J}^{\prime}$. Observing that $\check{\mathcal{Z}}^{(\mathbb{J}, \check{I})}$ and $\check{\mathcal{Z}}^{\left(\mathbb{J}^{\prime}, \check{\mathbb{I}}^{\prime}\right)}$ are compatible facet spans, and $\mathcal{P}_{\mathbb{J}}$ and $\mathcal{P}_{\mathbb{J}^{\prime}}$ are spanned by different primaries, we shall establish that $\mathcal{Z}^{(\mathrm{J}, \mathbb{I})}$ and $\mathcal{Z}^{\left(\mathbb{J}^{\prime}, \mathbb{I}^{\prime}\right)}$ are compatible by checking that $\mathcal{P}_{\mathbb{J}} \cap \mathcal{P}_{\mathbb{J}^{\prime}}, \mathcal{P}_{\mathbb{J}} \cap \check{\mathcal{P}}_{l}^{\left(\mathbb{J}^{\prime}, \check{\mathbb{I}}^{\prime}\right)}$, and $\check{\mathcal{P}}_{l}^{(\mathbb{J}, \check{I})} \cap \mathcal{P}_{\mathbb{J}^{\prime}}$, for all $l \in\langle M-3\rangle$, are faces for both of the parallelepipeds included in the corresponding intersections. Let $\mathcal{C}=\mathcal{P}_{\mathbb{J}} \cap \mathcal{P}_{\mathbb{J}^{\prime}}$. If $\mathcal{C}=\phi$, then $\mathcal{C}$ is clearly a common face of $\mathcal{P}_{\mathbb{J}}$ and $\mathcal{P}_{\mathbb{J}^{\prime}}$. Assume $\mathcal{C} \neq \phi$. Observing that $\mathcal{P}_{\mathbb{J}}$ and $\mathcal{P}_{\mathbb{J}^{\prime}}$ share with $\check{\mathcal{G}}$ the facets $\check{\mathcal{F}}_{\mathbb{J}}=\mathcal{P}_{\mathbb{J}} \bigcap \check{\mathcal{G}}$ and $\check{\mathcal{F}}_{\mathbb{J}^{\prime}}=\mathcal{P}_{\mathbb{J}^{\prime}} \bigcap \check{\mathcal{G}}$, respectively, we see that $\mathcal{P}_{\mathbb{J}}=\left\{\check{\mathbf{t}}+\zeta \operatorname{sgn}\left(\mathbf{u}_{\mathbb{J}_{0}}^{T} \mathbf{p}_{M}\right) \mathbf{p}_{M} \mid \check{\mathbf{t}} \in \check{\mathcal{F}}_{\mathbb{J}}, \zeta \in[0,1]\right\}$ and $\mathcal{P}_{\mathbb{J}^{\prime}}=\left\{\check{\mathbf{t}}+\zeta \operatorname{sgn}\left(\mathbf{u}_{\mathbb{J}_{0}}^{T} \mathbf{p}_{M}\right) \mathbf{p}_{M} \mid \check{\mathbf{t}} \in \check{\mathcal{F}}_{\mathbb{J}^{\prime}}, \zeta \in[0,1]\right\}$, therefore, $\mathcal{C}=\left\{\check{\mathbf{t}}+\zeta \operatorname{sgn}\left(\mathbf{u}_{\mathbb{J}_{0}}^{T} \mathbf{p}_{M}\right) \mathbf{p}_{M} \mid \check{\mathbf{t}} \in \check{\mathcal{F}}_{\mathbb{J}} \cap \check{\mathcal{F}}_{\mathbb{J}^{\prime}}, \zeta \in[0,1]\right\}$. Because $\check{\mathcal{F}}_{\mathbb{J}}$ and $\check{\mathcal{F}}_{\mathbb{J}^{\prime}}$ are gamut facets, it follows that $\check{\mathcal{F}}_{\mathbb{J}} \cap \mathcal{\mathcal { F }}_{\mathbb{J}^{\prime}}$ is a common face for $\check{\mathcal{F}}_{\mathbb{J}}$ and $\check{\mathcal{F}}_{\mathbb{J}^{\prime}}$, thus, a common face for $\mathcal{P}_{\mathbb{J}}$ and $\mathcal{P}_{\mathbb{J}}$, therefore, the intersection $\mathcal{C}$ is a common face for $\mathcal{P}_{\mathbb{J}}$ and $\mathcal{P}_{\mathbb{J}^{\prime}}$. Now, let $l \in\langle M-3\rangle$ and $\mathcal{C}=\mathcal{P}_{\mathbb{J}} \cap \check{\mathcal{P}}_{l}^{\left(\mathbb{J}^{\prime}, \check{I}^{\prime}\right)}$. We can see that for some $m \in\{1, M-3\}, \check{\mathcal{F}}_{\mathbb{J}}=\mathcal{P}_{\mathbb{J}} \bigcap \breve{\mathcal{G}}=\mathcal{P}_{\mathbb{J}} \bigcap \check{\mathcal{Z}}^{(\mathbb{I}, \mathbb{I})}=$ $\mathcal{P}_{\mathbb{J}} \cap \check{\mathcal{P}}_{m}^{(\mathbb{J}, \check{I})}$ is a common facet for $\mathcal{P}_{\mathbb{J}}, \check{\mathcal{P}}_{m}^{(\mathbb{J}, \tilde{\mathrm{I}})}$, and $\check{\mathcal{P}}_{m}^{(\mathbb{J}, \check{\mathrm{I}})}$ is the only parallelepiped in the gamut tiling $\mathcal{T}_{\tilde{\mathcal{K}}}$ for which 
$\check{\mathcal{F}}_{\mathbb{J}}$ is a facet. Because $\check{\mathcal{P}}_{m}^{(\mathbb{J}, \check{I})}, \check{\mathcal{P}}_{l}^{\left(\mathbb{J}^{\prime}, \check{I}^{\prime}\right)} \in \mathcal{T}_{\check{\mathcal{K}}}$, the intersection $\check{\mathcal{P}}_{m}^{(\mathbb{J}, \check{\mathbb{I}})} \cap \check{\mathcal{P}}_{l}^{\left(\mathbb{J}^{\prime}, \check{I}^{\prime}\right)}$ is a common face for $\check{\mathcal{P}}_{m}^{(\mathbb{J}, \tilde{\mathbb{I}})}$ and $\check{\mathcal{P}}_{l}^{\left(\mathbb{J}^{\prime}, \check{\mathbb{I}}^{\prime}\right)}$, and because $\mathcal{C} \subseteq \check{\mathcal{G}}$, we see that $\mathcal{C}=\mathcal{C} \cap \check{\mathcal{G}}=\left(\mathcal{P}_{\mathbb{J}} \cap \check{\mathcal{G}}\right) \cap \check{\mathcal{P}}_{l}^{\left(\mathbb{J}^{\prime}, \check{\mathbb{I}}^{\prime}\right)}=$ $\mathcal{P}_{\mathbb{J}} \cap \check{\mathcal{P}}_{m}^{(\widetilde{J}, \check{I})} \cap \check{\mathcal{P}}_{l}^{\left(\mathbb{J}^{\prime}, \check{\mathbb{I}}^{\prime}\right)}=\left(\mathcal{P}_{\mathbb{J}} \cap \check{\mathcal{G}}\right) \cap\left(\check{\mathcal{P}}_{m}^{(\mathbb{J}, \check{\mathbb{I}})} \cap \check{\mathcal{P}}_{l}^{\left(\mathbb{J}^{\prime}, \check{I}^{\prime}\right)}\right)=$ $\check{\mathcal{F}}_{\mathbb{J}} \cap\left(\check{\mathcal{P}}_{m}^{(\mathbb{J}, \check{I})} \cap \check{\mathcal{P}}_{l}^{\left(\mathbb{J}^{\prime}, \check{I}^{\prime}\right)}\right)$ is a common face for $\check{\mathcal{F}}_{\mathbb{J}}, \check{\mathcal{P}}_{m}^{(\mathbb{I}, \check{I})}$ and $\check{\mathcal{P}}_{l}^{\left(\mathbb{J}^{\prime}, \check{I}^{\prime}\right)}$, and therefore, a common face for $\mathcal{P}_{\mathbb{J}}$ and $\check{\mathcal{P}}_{l}^{\left(\mathbb{J}^{\prime}, \check{I}^{\prime}\right)}$. Using analogous arguments, we see that the intersection $\mathcal{C}=$ $\check{\mathcal{P}}_{l}^{(\mathbb{J}, \mathbb{I I})} \cap \mathcal{P}_{\mathbb{J}^{\prime}}$, is a common face for $\check{\mathcal{P}}_{l}^{(\mathbb{J}, \check{I})}$ and $\mathcal{P}_{\mathrm{J}}$. Therefore, the set of extensions $\mathcal{A}=\left\{\check{\mathcal{Z}}^{(\mathbb{I}, \check{I})} \cup \mathcal{P}_{\mathbb{J}} \mid \check{\mathcal{Z}}^{(\mathbb{I}, \check{I})} \in \check{\mathcal{K}}\right\}$ is a set of compatible facet spans for the $M$-primary system, with one facet span for each $\mathbb{J} \in \mathfrak{C}^{2}(\langle M-1\rangle)$, and associated collection of parallelepipeds $\mathcal{T}_{\mathcal{A}}=\mathcal{T}_{\breve{K}} \bigcup\left\{\mathcal{P}_{\mathbb{J}} \mid \mathbb{J} \in \mathfrak{C}^{2}(\langle M-1\rangle)\right\}$. Because $\mathscr{N}\left(\mathcal{T}_{\check{K}}\right)=\left(\begin{array}{c}M-1 \\ 3\end{array}\right)$ and no parallelepiped $\mathcal{P}_{\mathbb{J}}$ is in $\mathcal{T}_{\tilde{\mathcal{K}}}$, we see that $\mathscr{N}\left(\mathcal{I}_{\mathcal{A}}\right)=\mathscr{N}\left(\mathcal{T}_{\tilde{\mathcal{K}}}\right)+\left(\begin{array}{c}M-1 \\ 2\end{array}\right)=\left(\begin{array}{c}M \\ 3\end{array}\right)$, therefore, $\mathcal{A}$ is a maximal set, thus, there is a complete set $\mathcal{K}$ containing $\mathcal{A}$. Because $\mathcal{Z}^{\left(\mathbb{J}_{0}, \mathbb{I}_{0}\right)} \in \mathcal{A}$, it follows that $\mathcal{Z}^{\left(\mathbb{J}_{0}, \mathbb{I}_{0}\right)} \in \mathcal{K}$.

Finally, from Lemma 2, we see that $\mathcal{K}$ is a set of compatible facet spans iff $\tilde{\mathcal{K}}$ is a set of compatible facet spans. Observing that $\mathscr{N}\left(\mathcal{T}_{\tilde{K}}\right)=\mathscr{N}\left(\mathcal{T}_{\mathcal{K}}\right)$ we can conclude that $\mathcal{K}$ is maximal iff $\tilde{\mathcal{K}}$ is maximal, in which case, $\mathscr{N}\left(\mathcal{T}_{\tilde{\mathcal{K}}}\right)=\mathscr{N}\left(\mathcal{T}_{\mathcal{K}}\right)=\left(\begin{array}{c}K \\ 3\end{array}\right)$.

\section{E. Proof of Theorem 2}

Let $\mathbf{t} \in \mathcal{G}$ and $\boldsymbol{\omega} \in \Omega(\mathbf{t})$. Assume first that $\boldsymbol{\omega} \in \mathcal{V}_{\Omega(\mathbf{t})}$, then, from Theorem $1, \omega$ has at most three components in $(0,1)$, and we denote by $\mathbb{P} \in \mathfrak{C}^{3}(\langle K\rangle)$ a set of indices that includes these components. We then have the decomposition $\boldsymbol{\omega}=\boldsymbol{\alpha}+\mathbf{I}_{K}[\mathbb{P}] \boldsymbol{\nu}$, where $\boldsymbol{\nu}=\left[\omega_{\mathbb{P}[1]}, \omega_{\mathbb{P}[2]}, \omega_{\mathbb{P}[3]}\right]^{T} \in[0,1]^{3}$, and $\boldsymbol{\alpha} \in\{0,1\}^{K}$, with $\alpha_{\mathbb{P}[1]}=\alpha_{\mathbb{P}[2]}=\alpha_{\mathbb{P}[3]}=0$ and $\alpha_{k}=\omega_{k}$, for $k \in\langle K\rangle \backslash \mathbb{P}$. Then, $\mathbf{t}=\mathbf{P} \boldsymbol{\omega}+\mathbf{t}_{\mathbf{0}}=\mathbf{P}[\mathbb{P}] \boldsymbol{\nu}+\left(\mathbf{P} \boldsymbol{\alpha}+\mathbf{t}_{\mathbf{0}}\right)=$ $\mathbf{P}[\mathbb{P}] \boldsymbol{\nu}+\mathbf{v}$, where $\mathbf{v}=\mathbf{P} \boldsymbol{\alpha}+\mathbf{t}_{\mathbf{0}}$. Because the primaries $\mathbf{P}[\mathbb{P}]$ are linearly independent, $\boldsymbol{\nu}=\mathbf{P}^{-1}[\mathbb{P}](\mathbf{t}-\mathbf{v})$ and $\mathbf{t}$ is in the parallelepiped $\mathcal{P}(\mathbf{v}, \mathbf{P}[\mathbb{P}]) \subseteq \mathcal{G}$, which we will show belongs to a gamut tiling. Let $\mathbb{J} \in \mathfrak{C}^{2}(\langle\mathbb{P}\rangle)$ and let $\mathbb{I}_{1}=\left\{k \in\langle K\rangle \backslash \mathbb{P} \mid \chi^{+}\left(\mathbf{u}_{\mathbb{J}}^{T} \mathbf{p}_{k}\right)=\alpha_{k}\right\}, \mathbb{I}_{2}=\{k \in$ $\left.\langle K\rangle \backslash \mathbb{P} \mid \chi^{-}\left(\mathbf{u}_{\mathbb{J}}^{T} \mathbf{p}_{k}\right)=\alpha_{k}\right\}, \mathbb{I}=\left[\mathbb{I}_{1}, \mathbb{P} \backslash \mathbb{J}, \mathbb{I}_{2}\right]$, and $l=\mathscr{N}\left(\mathbb{I}_{1}\right)+1$ (where the orderings within the subsequences $\mathbb{I}_{1}$ and $\mathbb{I}_{2}$ can be chosen arbitrarily); note that $1 \leq l \leq$ $K-2$. Then, through algebraic manipulation, we can see that $\mathcal{P}_{l}^{(\mathbb{J}, \mathbb{I})}$, the $l^{\text {th }}$ parallelepiped of the facet span $\mathcal{Z}^{(\mathbb{J}, \mathbb{I})}$, is spanned by primaries $\mathbf{P}[\mathbb{P}]$, and has the origin $\mathbf{c}_{l}^{(\mathbb{I}, \mathbb{I})}=$ $\mathbf{t}_{\mathbf{0}}+\sum_{i=1}^{l-1} \chi^{+}\left(\mathbf{u}_{\mathbb{J}}^{T} \mathbf{p}_{\mathbb{I}[i]}\right) \mathbf{p}_{\mathbb{I}[i]}+\sum_{i=l+1}^{K-2} \chi^{-}\left(\mathbf{u}_{\mathbb{J}}^{T} \mathbf{p}_{\mathbb{I}[i]}\right) \mathbf{p}_{\mathbb{I}[i]}=$ $\mathbf{t}_{\mathbf{0}}+\sum_{m \in\langle K\rangle \backslash \mathbb{P}} \alpha_{m} \mathbf{p}_{m}=\mathbf{t}_{\mathbf{0}}+\sum_{m=1}^{K} \alpha_{m} \mathbf{p}_{m}=\mathbf{P} \boldsymbol{\alpha}+\mathbf{t}_{\mathbf{0}}=\mathbf{v}$, therefore, $\mathcal{P}_{l}^{(\mathbb{J}, \mathbb{I})}=\mathcal{P}(\mathbf{v}, \mathbf{P}[\mathbb{P}])$. Now, from Lemma $4, \mathcal{Z}^{(\mathbb{I}, \mathbb{I})} \in$ $\mathcal{K}$, for some complete set $\mathcal{K}$, with an associated gamut tiling $\mathcal{T}_{\mathcal{K}}$ and tiling $\operatorname{CCF} \mathscr{C}_{\mathcal{I}_{\mathcal{K}}}$. Because $\mathcal{P}(\mathbf{v}, \mathbf{P}[\mathbb{P}]) \in \mathcal{T}_{\mathcal{K}}$, we see that $\boldsymbol{\omega}=\boldsymbol{\alpha}+\mathbf{I}_{K}[\mathbb{P}] \boldsymbol{\nu}=\boldsymbol{\alpha}+\mathbf{I}_{K}[\mathbb{P}](\mathbf{P}[\mathbb{P}])^{-1}(\mathbf{t}-\mathbf{v})=\mathscr{C}_{\mathcal{T}_{\mathcal{K}}}(\mathbf{t})$, therefore, $\boldsymbol{\omega}$ is a tiling control vector.

To show the converse, assume now that $\boldsymbol{\omega}$ is a tiling control vector. Then, there is a tiling $\mathcal{T}$ with tiling $\mathrm{CCF}$ $\mathscr{C}_{\mathcal{T}}$ and a parallelepiped $\mathcal{P} \in \mathcal{T}$ spanned by $\mathbf{P}[\mathbb{P}]$, with $\mathbb{P} \in \mathfrak{C}^{3}(\langle K\rangle)$, with origin $\mathbf{v}$, such that $\mathbf{t} \in \mathcal{P}$ and $\boldsymbol{\omega}=$ $\mathscr{C}_{\mathcal{T}}(\mathbf{t})=\boldsymbol{\alpha}+\mathbf{I}_{K}[\mathbb{P}](\mathbf{P}[\mathbb{P}])^{-1}(\mathbf{t}-\mathbf{v})$, where $\boldsymbol{\alpha} \in\{0,1\}^{K}$ is a control vector for $\mathbf{v}$, with $\alpha_{\mathbb{P}[1]}=\alpha_{\mathbb{P}[2]}=\alpha_{\mathbb{P}[3]}=0$. Therefore, $\omega_{k}=\alpha_{k}$, for $k \in\langle K\rangle \backslash \mathbb{P}$, and only the three vector components $\omega_{\mathbb{P}[1]}, \omega_{\mathbb{P}[2]}, \omega_{\mathbb{P}[3]}$ may be in $(0,1)$, so from Theorem 1, $\boldsymbol{\omega} \in \mathcal{V}_{\Omega(\mathbf{t})}$.

\section{APPENDIX C \\ PRIMARY SySTEMS USED IN EXAMPLES}

Table $\mathrm{V}$ lists the tristimulus values corresponding to the columns of the primary matrices used as examples in the paper and in the subsequent appendices. The four primary system $\mathbf{P}_{e}^{(4)}$ is identical to the one used in the companion Part I Paper [1] and the five-primary system $\mathbf{P}_{e}^{(5)}$ is an extension of the four primary system $\mathbf{P}_{e}^{(4)}$, with the fifth column as an added primary, viz. $\mathbf{P}_{e}^{(5)}=\left[\mathbf{P}_{e}^{(4)} \mid \mathbf{p}_{5}\right]$. Both $\mathbf{P}_{e}^{(4)}$ and $\mathbf{P}_{e}^{(5)}$ are chosen in order to generate convenient renderings of gamuts and facet spans for the visualizations and also use a value of $\mathbf{t}_{\mathbf{0}}=[35,35,35]^{T}$ to improve the visualizations by distancing the axes from the gamut facets and edges. The five-primary system $\mathbf{P}_{w}^{(5)}$ is chosen to illustrate that not all MCS vertices are progressive tiling control vectors and is obtained as an extension of the four primary design from the companion Part I Paper [1, Sect. C], which was designed to optimize gamut volume under a total power constraint. In particular, the fifth primary of $\mathbf{P}_{w}^{(5)}$ is defined as $\mathbf{p}_{5}=(1 / 4) \sum_{k=1}^{4} \mathbf{p}_{k}$, so $\mathbf{p}_{5}$ has the chromaticity of the display white $\mathbf{t}_{\mathbf{1}}$ and accounts for $20 \%$ of the luminance of $\mathbf{t}_{\mathbf{1}}$. For aiding visualization, the multiprimary system $\mathbf{P}_{w}^{(5)}$ is used in conjunction with $\mathbf{t}_{\mathbf{0}}=[15,15,15]^{T}$. The six primary system $\mathbf{P}_{\mathscr{V}}^{(6)}$ is the six primary design from the companion Part I Paper [1, Appendix. C], designed to optimize gamut volume under a total power constraint (and has an associated value of $\left.\mathbf{t}_{\mathbf{0}}=[0,0,0]^{T}\right)$.

\begin{tabular}{lrrrrrrr}
\hline & & \multicolumn{1}{c}{$\mathbf{p}_{1}$} & \multicolumn{1}{c}{$\mathbf{p}_{2}$} & \multicolumn{1}{c}{$\mathbf{p}_{3}$} & \multicolumn{1}{c}{$\mathbf{p}_{4}$} & $\mathbf{p}_{5}$ & $\mathbf{p}_{6}$ \\
\hline & $\mathrm{X}$ & 0.03 & 1.74 & 118.69 & 86.47 & & \\
$\mathbf{P}_{e}^{(4)}$ & $\mathrm{Y}$ & 20.32 & 85.49 & 3.65 & 76.53 & & \\
& $\mathrm{Z}$ & 114.68 & 58.16 & 22.78 & 31.18 & & \\
\hline & $\mathrm{X}$ & 0.03 & 1.74 & 118.69 & 86.47 & 10.30 & \\
$\mathbf{P}_{e}^{(5)}$ & $\mathrm{Y}$ & 20.32 & 85.49 & 3.65 & 76.53 & 35.03 & \\
& $\mathrm{Z}$ & 114.68 & 58.16 & 22.78 & 31.18 & 14.79 & \\
\hline & $\mathrm{X}$ & 16.88 & 0.78 & 5.67 & 71.82 & 23.79 & \\
$\mathbf{P}_{w}^{(5)}$ & $\mathrm{Y}$ & 0.49 & 19.81 & 53.59 & 26.11 & 25.00 & \\
& $\mathrm{Z}$ & 80.98 & 22.86 & 5.17 & 0.00 & 27.25 & \\
\hline & $\mathrm{X}$ & 14.56 & 2.48 & 0.14 & 2.88 & 5.24 & 69.87 \\
$\mathbf{P}_{\mathscr{V}}^{(6)}$ & $\mathrm{Y}$ & 0.42 & 6.57 & 18.63 & 34.20 & 14.79 & 25.40 \\
& $\mathrm{Z}$ & 69.62 & 25.31 & 9.70 & 4.11 & 0.22 & 0.00 \\
\hline & & & & & & &
\end{tabular}

TABLE V

CIE XYZ TRISTIMULI OF THE PRIMARIES FOR THE MULTIPRIMARY SYSTEMS USED FOR THE EXAMPLES

\section{APPENDIX D}

\section{CBS-BASED MCS VISUALIZATION: PARAMETERS \& SYMMETRY}

The CBS based visualization strategy described in Section III-A relies on the selection of an orthonormal basis $\mathbf{B}$ for the CBS. For a primary matrix $\mathbf{P}$, we obtain $\mathbf{B}$ conveniently from the SVD decomposition [5, pp. 411], $\mathbf{P}=\mathbf{U S V}^{T}$ of $\mathbf{P}$, 
where $\mathbf{S}$ is a $3 \times K$ diagonal matrix containing the singular values of $\mathbf{P}$, and $\mathbf{U}, \mathbf{V}$ are $3 \times 3$ and $K \times K$ orthogonal matrices. Denoting $\mathbf{V}=\left[\mathbf{v}_{1}, \ldots, \mathbf{v}_{K}\right]$, we note that the last $(K-3)$ columns of $\mathbf{V}$ form an orthonormal basis for the null space (CBS) of $\mathbf{P}$ [27]. Therefore, we set the basis matrix $\mathbf{B} \stackrel{\text { def }}{=}\left[\mathbf{b}_{1}, \ldots, \mathbf{b}_{K-3}\right]$, with $\mathbf{b}_{1}=\mathbf{v}_{4}, \ldots, \mathbf{b}_{K-3}=\mathbf{v}_{K}$. The bases for the primary systems we obtain for each primary system in Section C using this procedure are listed in Table VI.

\begin{tabular}{rrrr}
\hline & \multicolumn{1}{c}{$\mathbf{b}_{1}$} & \multicolumn{1}{c}{$\mathbf{b}_{2}$} & $\mathbf{b}_{3}$ \\
\hline & -0.25 & -0.23 & \\
$\mathbf{B}_{w}^{(5)}$ & 0.89 & -0.21 & \\
& -0.38 & -0.23 & \\
& 0.06 & -0.22 & \\
& 0.07 & 0.90 & \\
\hline & 0.27 & 0.11 & -0.16 \\
$\mathbf{B}_{\mathscr{V}}^{(6)}$ & -0.63 & -0.19 & 0.66 \\
& -0.49 & -0.24 & -0.70 \\
& 0.50 & -0.17 & 0.25 \\
& -0.20 & 0.92 & 0.02 \\
& -0.04 & -0.08 & -0.00 \\
\hline
\end{tabular}

TABLE VI

ORTHONORMAL BASES OF THE CBS OF THE MULTIPRIMARY SYSTEMS $\mathbf{P}_{w}^{(5)} \operatorname{AND} \mathbf{P}_{\mathscr{V}}^{(6)}$

Figure 2 described how the MCS polytope can be conveniently visualized using it CBS representation $\Xi(\mathbf{t})$ and demonstrated examples for the five and six primary systems $\mathbf{P}_{w}^{(5)}$ and $\mathbf{P}_{\mathscr{V}}^{(6)}$. The visualizations presented in Fig. 2 for these two systems used the bases specified in Section $\mathrm{C}$ and corresponded to the tristimulus $\mathbf{t}=[51.47,75.35,101.18]^{T}$ for the five primary system $\mathbf{P}_{w}^{(5)}$ and the tristimulus $\mathbf{t}=$ $[89.53,82.42,97.20]^{T}$; for the six primary system $\mathbf{P}_{\mathscr{V}}^{(6)}$, in both cases, $\mathbf{t}_{\mathbf{0}}=\mathbf{0}$. We note here that MCSs also exhibit symmetry that is induced by the symmetry of the control space and the gamut. Specifically, the feasible control space $[0,1]^{K}$ is a polytope that is centrally symmetric [2] about the center $\boldsymbol{\alpha}_{c}=(1 / 2) \mathbf{1}$, i.e., for any $\boldsymbol{\nu} \in \mathbb{R}^{K}, \boldsymbol{\alpha}_{c}+\boldsymbol{\nu} \in[0,1]^{K}$ iff $\boldsymbol{\alpha}_{c}-\boldsymbol{\nu} \in[0,1]^{K}$. Correspondingly, the control vectors $\boldsymbol{\alpha}$ and $\tilde{\boldsymbol{\alpha}} \stackrel{\text { def }}{=} \mathbf{1}-\boldsymbol{\alpha}$ are referred to as the (centrally) symmetric images of each other. Because $\mathcal{G}$ is an affine mapping of $[0,1]^{K}$ into $\mathbb{R}^{3}, \mathcal{G}$ is also centrally symmetric [2], with center $\mathbf{t}_{c}=(1 / 2)\left(\mathbf{t}_{\mathbf{0}}+\mathbf{t}_{\mathbf{1}}\right)$, and it follows that the symmetric image of $\mathbf{t} \in \mathcal{G}$ is $\tilde{\mathbf{t}} \stackrel{\text { def }}{=} \mathbf{t}_{\mathbf{1}}-\left(\mathbf{t}-\mathbf{t}_{\mathbf{0}}\right)$. Now, observing that for $\mathbf{t} \in \mathcal{G}, \boldsymbol{\alpha} \in \Omega(\mathbf{t})$ iff $\mathbf{t}=\mathbf{t}_{\mathbf{0}}+\mathbf{P} \boldsymbol{\alpha}$ and $\boldsymbol{\alpha} \in[0,1]^{K}$, iff $\tilde{\mathbf{t}}=\mathbf{t}_{\mathbf{0}}+\mathbf{P}(\mathbf{1}-\boldsymbol{\alpha})=\mathbf{t}_{\mathbf{0}}+\mathbf{P} \tilde{\boldsymbol{\alpha}} \in \mathcal{G}$, iff $\tilde{\boldsymbol{\alpha}} \in \Omega(\tilde{\mathbf{t}})$, where $\tilde{\boldsymbol{\alpha}} \stackrel{\text { def }}{=} \mathbf{1}-\boldsymbol{\alpha}$ is the symmetric image of $\boldsymbol{\alpha}$. It follows that $\Omega(\tilde{\mathbf{t}})=\{\tilde{\boldsymbol{\alpha}} \mid \boldsymbol{\alpha} \in \Omega(\mathbf{t})\}$ is the symmetric image of $\Omega(\mathbf{t})$. Similarly, we see that the coordinate representation of $[0,1]^{K}$ in terms of the CBS orthonormal basis $\mathbf{B}$ is the zonotope $\mathcal{A}=\left\{\mathbf{B}^{T} \boldsymbol{\alpha} \mid \boldsymbol{\alpha} \in[0,1]^{K}\right\}$ in $\mathbb{R}^{K-3}$, which is centrally symmetric with center $\mathbf{B}^{T} \boldsymbol{\alpha}_{c}$, so the symmetric image of $\boldsymbol{\beta} \in \mathcal{A}$ is $\tilde{\boldsymbol{\beta}}=\mathbf{B}^{T} \mathbf{1}-\boldsymbol{\beta}$. Therefore, the coordinate representation of $\Omega(\tilde{\mathbf{t}})$ with respect the CBS basis $\mathbf{B}$ is $\Xi(\tilde{\mathbf{t}})=\left\{\mathbf{B}^{T} \tilde{\boldsymbol{\alpha}} \mid \boldsymbol{\alpha} \in \Omega(\mathbf{t})\right\}=\left\{\mathbf{B}^{T}(\mathbf{1}-\boldsymbol{\alpha}) \mid \boldsymbol{\alpha} \in \Omega(\mathbf{t})\right\}=$ $\left\{\mathbf{B}^{T} \mathbf{1}-\mathbf{B}^{T} \boldsymbol{\alpha} \mid \boldsymbol{\alpha} \in \Omega(\mathbf{t})\right\}\left\{\mathbf{B}^{T} \mathbf{1}-\boldsymbol{\beta} \mid \boldsymbol{\beta} \in \Xi(\mathbf{t})\right\}$, which is the symmetric image of $\Xi(\mathbf{t})$. Figure 10 illustrates

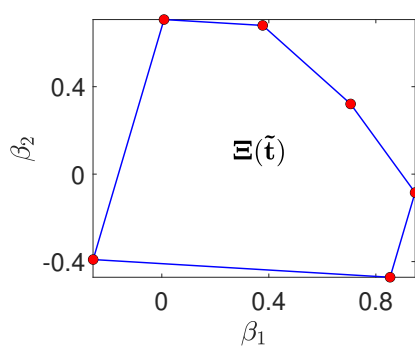

(a) $K=5$

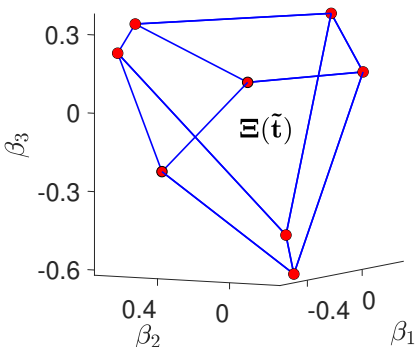

(b) $K=6$
Fig. 10. MCS polytopes $\Xi(\tilde{\mathbf{t}})$ for the symmetric tristimuli corresponding to the MCS polytopes shown in Fig. 2 for the $K=5,6$ primary systems $\mathbf{P}_{w}^{(5)}, \mathbf{P}_{\mathscr{V}}^{(6)}$. The MCS polytopes are shown using $(K-3)$-dimensional CBS coordinate representations developed in Section III-A.

the MCS for the symmetric tristimuli $\tilde{\mathbf{t}}$ corresponding to the tristimuli $\mathbf{t}$ shown in Fig. 2, where it can be appreciated that the each of the symmetric images $\Xi(\tilde{\mathbf{t}})$ can be obtained by flipping the corresponding $\Xi(\mathbf{t})$ about the coordinate axes and translating the resulting polytope, as indicated by the preceding mathematical relation.

\section{APPENDIX E}

\section{PARALLELEPIPED INTERSECTION CHECK}

The strong-compatibility check introduced in Algorithm 2 relies on checking whether or not two parallelepipeds from two different facet spans intersect each other. In this section, we describe a method for efficiently performing this check by exploiting the characterization of parallelepipeds in Lemma 1 of the companion Part I Paper [1]. We refer the reader to Lemma 1 and to Section II of the Part I paper, for the results and notation that we use in the rest of this section.

For the parallelepiped $\mathcal{P}=\mathcal{P}(\mathbf{v}, \mathbf{A})$ spanned by a $3 \times 3$ nonsingular matrix $\mathbf{A}$ and origin $\mathbf{v} \in \mathbb{R}^{3}$, we denote by $\mathcal{W}_{\mathcal{P}}$ the set of edges of $\mathcal{P}$. Each edge of $\mathcal{P}$ is a line segment $\mathcal{E}=\mathcal{E}(\mathbf{w}, \mathbf{a})$, where the vector $\mathbf{a} \in \mathbb{R}^{3}$ is a column of $\mathbf{A}$ and $\mathbf{w} \in \mathbb{R}^{3}$ is a vertex of $\mathcal{P}$. Then, the following lemma follows immediately.

Lemma 5. For a pair of parallelepipeds $\mathcal{P}$ and $\mathcal{P}^{\prime}$ with sets of edges $\mathcal{W}_{\mathcal{P}}$ and $\mathcal{W}_{\mathcal{P}^{\prime}}$, respectively, the following properties hold:

1) $\mathcal{P} \cap \mathcal{P}^{\prime}=\phi$, iff, $\mathcal{P} \cap \mathcal{E}^{\prime}=\mathcal{P}^{\prime} \cap \mathcal{E}=\phi$, for every $\mathcal{E} \in \mathcal{W}_{\mathcal{P}}$ and every $\mathcal{E}^{\prime} \in \mathcal{W}_{\mathcal{P}^{\prime}}$.

2) $\operatorname{Int}(\mathcal{P}) \cap \operatorname{Int}\left(\mathcal{P}^{\prime}\right)=\phi$, iff, $\operatorname{Int}(\mathcal{P}) \cap \operatorname{Int}\left(\mathcal{E}^{\prime}\right)=\operatorname{Int}\left(\mathcal{P}^{\prime}\right) \cap$ $\operatorname{Int}(\mathcal{E})=\phi$, for every $\mathcal{E} \in \mathcal{W}_{\mathcal{P}}$ and every $\mathcal{E}^{\prime} \in \mathcal{W}_{\mathcal{P}^{\prime}}$.

Because a parallelepiped has 12 edges, Lemma 5 allows one to perform the parallelepiped intersection check as the evaluation of 24 different intersection checks between a line segment and a parallelepiped. Thus, the three-dimensional problem of checking the intersection of parallelepipeds is simplified to the evaluation of one-dimensional intersections.

For a parallelepiped $\mathcal{P}$, a line segment $\mathcal{E}(\mathbf{w}, \mathbf{q})$ and $\mathbf{t} \in \mathbb{R}^{3}$, we see that $\mathbf{t} \in \mathcal{E}(\mathbf{w}, \mathbf{q})$, iff, $\mathbf{t}=\mathbf{w}+\zeta \mathbf{q}$ for some $\zeta \in[0,1]$, and from Clause 1 of Lemma 1 in the Part I Paper [1], we see that $\mathbf{t} \in \mathcal{P}$, iff, $\tau^{\mathbb{J}} \leq \mathbf{u}_{\mathbb{J}}^{T} \mathbf{t} \leq \nu^{\mathbb{J}}$, for all $\mathbb{J} \in \mathfrak{C}^{2}(\langle 3\rangle)$. Therefore, $\mathcal{P} \cap \mathcal{E} \neq \phi$ iff there exists a $\zeta \in[0,1]$ such that 
$\tau^{\mathbb{J}} \leq \mathbf{u}_{\mathbb{J}}^{T} \mathbf{w}+\zeta \mathbf{u}_{\mathbb{J}}^{T} \mathbf{q} \leq \nu^{\mathbb{J}}$, for all $\mathbb{J} \in \mathfrak{C}^{2}(\langle 3\rangle)$. Now, for each index pair $\mathbb{J} \in \mathfrak{C}^{2}(\langle 3\rangle)$, and for $\mathbf{w}, \mathbf{q} \in \mathbb{R}^{3}$, with $\mathbf{u}_{\mathbb{J}}^{T} \mathbf{q} \neq \mathbf{0}$, we define the functions,

$$
\begin{aligned}
& \mathscr{L}_{\mathbb{J}}(\mathbf{w}, \mathbf{q})=\frac{\chi^{+}\left(\mathbf{u}_{\mathbb{J}}^{T} \mathbf{q}\right) \tau^{\mathbb{J}}+\chi^{-}\left(\mathbf{u}_{\mathbb{J}}^{T} \mathbf{q}\right) \nu^{\mathbb{J}}-\mathbf{u}_{\mathbb{J}}^{T} \mathbf{w}}{\mathbf{u}_{\mathbb{J}}^{T} \mathbf{q}}, \\
& \mathscr{U}_{\mathbb{J}}(\mathbf{w}, \mathbf{q})=\frac{\chi^{-}\left(\mathbf{u}_{\mathbb{J}}^{T} \mathbf{q}\right) \tau^{\mathbb{J}}+\chi^{+}\left(\mathbf{u}_{\mathbb{J}}^{T} \mathbf{q}\right) \nu^{\mathbb{J}}-\mathbf{u}_{\mathbb{J}}^{T} \mathbf{w}}{\mathbf{u}_{\mathbb{J}}^{T} \mathbf{q}},
\end{aligned}
$$

which we use to express necessary and sufficient conditions for a non-empty intersection of a parallelepiped and a line segment in the following lemma.

Lemma 6. For a parallelepiped $\mathcal{P}$ and a line segment $\mathcal{E}(\mathbf{w}, \mathbf{q})$, let $\mathfrak{J}_{\mathbf{q}}=\left\{\mathbb{J} \in \mathfrak{C}^{2}(\langle 3\rangle) \mid \mathbf{u}_{\mathbb{J}}^{T} \mathbf{q}=0\right\}$. Then,

1) $\mathcal{P} \cap \mathcal{E} \neq \phi$, iff, (a) for all $\mathbb{J} \in \mathfrak{J}_{\mathbf{q}}, \tau^{\mathbb{J}} \leq \mathbf{u}_{\mathbb{J}}^{T} \mathbf{w} \leq \nu^{\mathbb{J}}$, and (b) for all $\mathbb{J} \in \mathfrak{C}^{2}(\langle 3\rangle) \backslash \mathfrak{J}_{\mathbf{q}}, \mathscr{L}_{\mathbb{J}}(\mathbf{w}, \mathbf{q}) \leq 1,0 \leq$ $\mathscr{U}_{\mathbb{J}}(\mathbf{w}, \mathbf{q})$ and $\mathscr{L}_{\mathbb{J}}(\mathbf{w}, \mathbf{q}) \leq \mathscr{U}_{\mathbb{J}}(\mathbf{w}, \mathbf{q})$.

2) $\operatorname{Int}(\mathcal{P}) \cap \operatorname{Int}(\mathcal{E}) \neq \phi$, iff, (a) for all $\mathbb{J} \in \mathfrak{J}_{\mathbf{q}}, \tau^{\mathbb{J}}<\mathbf{u}_{\mathbb{J}}^{T} \mathbf{w}<$ $\nu^{\mathbb{J}}$, and (b) for all $\mathbb{J} \in \mathfrak{C}^{2}(\langle 3\rangle) \backslash \mathfrak{J}_{\mathbf{q}}, \mathscr{L}_{\mathbb{J}}(\mathbf{w}, \mathbf{q})<1$, $0<\mathscr{U}_{\mathbb{J}}(\mathbf{w}, \mathbf{q})$ and $\mathscr{L}_{\mathbb{J}}(\mathbf{w}, \mathbf{q})<\mathscr{U}_{\mathbb{J}}(\mathbf{w}, \mathbf{q})$.

Proof: We start by showing Clause 1 . Note that $\mathcal{P} \cap \mathcal{E} \neq \phi$, iff, there is a $\mathbf{t} \in \mathbb{R}^{3}$ such that $\mathbf{t} \in \mathcal{P} \cap \mathcal{E}$, iff, there is $\zeta \in[0,1]$ such that $\tau^{\mathbb{J}} \leq \mathbf{u}_{\mathbb{J}}^{T} \mathbf{w}+\zeta \mathbf{u}_{\mathbb{J}}^{T} \mathbf{q} \leq \nu^{\mathbb{J}}$, for all $\mathbb{J} \in \mathfrak{C}^{2}(\langle 3\rangle)$, iff:

- for all $\mathbb{J} \in \mathfrak{J}_{\mathbf{q}}, \tau^{\mathbb{J}} \leq \mathbf{u}_{\mathbb{J}}^{T} \mathbf{w} \leq \nu^{\mathbb{J}}$, and

- for all $\mathbb{J} \in \mathfrak{C}^{2}(\langle 3\rangle) \backslash \mathfrak{J}_{\mathbf{q}}, \tau^{\mathbb{J}}-\mathbf{u}_{\mathbb{J}}^{T} \mathbf{w} \leq \zeta \mathbf{u}_{\mathbb{J}}^{T} \mathbf{q} \leq \nu^{\mathbb{J}}-$ $\mathbf{u}_{\mathbb{J}}^{T} \mathbf{w}$, i.e., $\mathscr{L}_{\mathbb{J}}(\mathbf{w}, \mathbf{q}) \leq \zeta \leq \mathscr{U}_{\mathbb{J}}(\mathbf{w}, \mathbf{q})$, and $\zeta \in[0,1]$. Which is possible iff, $\mathscr{L}_{\mathbb{J}}(\mathbf{w}, \mathbf{q}) \leq 1,0 \leq \mathscr{U}_{\mathbb{J}}(\mathbf{w}, \mathbf{q})$ and $\mathscr{L}_{\mathbb{J}}(\mathbf{w}, \mathbf{q}) \leq \mathscr{U}_{\mathbb{J}}(\mathbf{w}, \mathbf{q})$.

Because $\operatorname{Int}(\mathcal{P}) \cap \operatorname{Int}(\mathcal{E}) \neq \phi$ iff, there is $\mathbf{t} \in \mathbb{R}^{3}$ such that $\mathbf{t} \in$ $\mathcal{P} \cap \mathcal{E}$, iff, there is $\zeta \in(0,1)$ such that $\tau^{\mathbb{J}}<\mathbf{u}_{\mathbb{J}}^{T} \mathbf{w}+\zeta \mathbf{u}_{\mathbb{J}}^{T} \mathbf{q}<\nu^{\mathbb{J}}$, for all $\mathbb{J} \in \mathfrak{C}^{2}(\langle 3\rangle)$, analogous arguments establish Clause 2 .

Now, based on Lemma 5 and Lemma 6, checking whether two parallelepipeds or their interiors intersect can be performed as stated in Algorithm 3.

\section{REFERENCES}

[1] G. Sharma and C. E. Rodríguez-Pardo, "Geometry of multiprimary display colors I: Gamut and color control," submitted for review.

[2] G. M. Ziegler, Lectures on polytopes, ser. Graduate Texts in Mathematics. New York, NY: Springer Science \& Business Media, 2007, vol. 152.

[3] S. R. Lay, Convex sets and their applications. Malabar, FL: Krieger Publishing Company, 1992.

[4] C. E. Rodríguez-Pardo and G. Sharma, "Calibration sets for multiprimary displays: Representation, visualization, and applications," in Proc. IS\&T/SID 22nd Color and Imaging Conference, Boston, MA, 3-7 Nov. 2014, pp. 171-179.

[5] C. Meyer, Matrix analysis and applied linear algebra. Philadephia, PA: SIAM, 2000.

[6] G. Sharma and H. J. Trussell, "Digital color imaging," IEEE Trans. Image Proc., vol. 6, no. 7, pp. 901-932, Jul. 1997.

[7] J. B. Cohen, Ed., Visual Color and Color Mixture: The Fundamental Color Space. Urbana, IL: University of Illinois Press, 2001.

[8] G. Wyszecki and W. S. Stiles, Color Science: Concepts and Methods, Quantitative Data and Formulae, 2nd ed. New York: John Wiley \& Sons, Inc., 1982.

[9] B. Chilton and H. Coxeter, "Polar zonohedra," Amer. Math. Monthly, pp. 946-951, 1963.

[10] G. W. Hart, "A color-matching dissection of the rhombic enneacontahedron," Symmetry: Culture and Science, vol. 11, pp. 183-199, 2000. [Online]. Available: http://www.georgehart.com/dissectre/dissect-re.htm

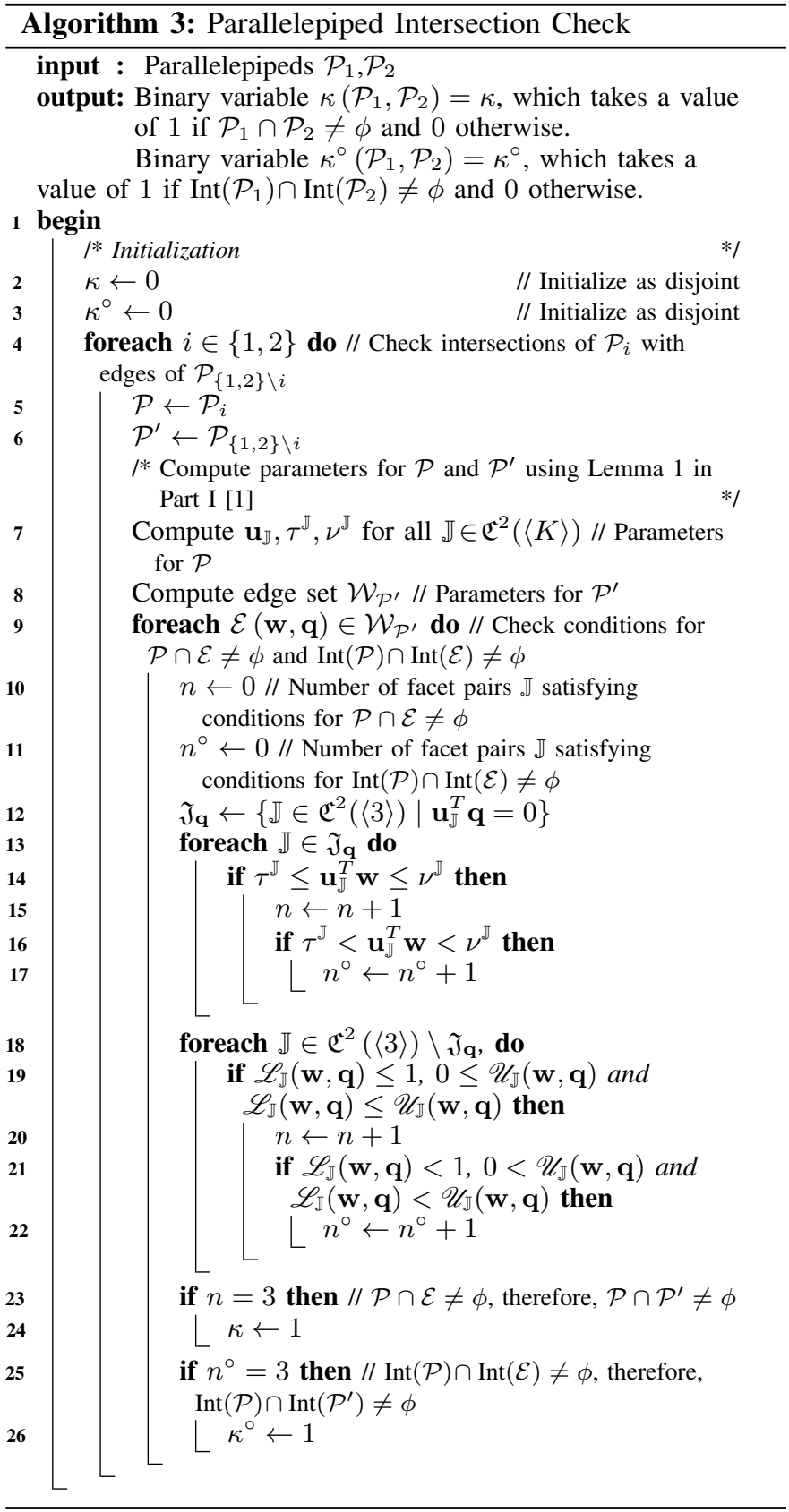

[11] H. Coxeter, "The classification of zonohedra by means of projective diagrams," J. Math. Pures Appl. (9), vol. 41, pp. 137-156, 1962.

[12] P. Centore, "Non-metamerism of boundary colors in multi-primary displays," J. Soc. Inf. Display, vol. 20, no. 4, pp. 214-220, 2012.

[13] F. Konig, K. Ohsawa, M. Yamaguchia, N. Ohyama, and B. Hill, "A multiprimary display: Optimized control values for displaying tristimulus values," in Proc. IS\&T/SID PICS 2002: Image Processing, Image Quality, Image Capture Systems Conference. Society for Imaging Science \& Technology, 2002, pp. 215-220.

[14] A. A. Goldstein, "Convex programming in Hilbert space," Bull. Amer. Math. Soc., vol. 70, no. 5, pp. 709-710, 1964.

[15] P. H. Calamai and J. J. Moré, "Projected gradient methods for linearly constrained problems," Math. Program., vol. 39, no. 1, pp. 93-116, 1987.

[16] C. E. Rodríguez-Pardo and G. Sharma, "Color control functions for multiprimary displays II: Variational robustness optimization," IEEE Trans. Image Proc., vol. 29, no. 1, pp. 1164-1176, 2020.

[17] M. Takaya, K. Ito, G. Ohashi, and Y. Shimodaira, "Color-conversion method for a multi-primary display to reduce power consumption and conversion time," J. Soc. Inf. Display, vol. 13, no. 8, pp. 685-690, 2012.

[18] P. Centore, "Minimal-energy control sequences for linear multi-primary 
displays," J. Imaging Sci. and Tech., vol. 59, no. 5, pp. 50 502-1-10, 2015.

[19] D.-W. Kang, Y.-H. Cho, Y.-T. Kim, W.-H. Choe, and Y.-H. Ha, "Multiprimary decomposition method based on a three-dimensional look-up table in linearized LAB space for reproduction of smooth tonal change," J. Imaging Sci. and Tech., vol. 50, no. 4, pp. 357-367, 2006.

[20] H. Motomura, "Color conversion for a multi-primary display using linear interpolation on equi-luminance plane method (LIQUID)," J. Soc. Inf. Display, vol. 11, no. 2, pp. 371-378, 2003.

[21] H. Kanazawa, M. Mitsui, M. Yamaguchi, H. Haneishi, and N. Ohyama, "Color conversion for multi-primary displays using a spherical average method," in Proc. IS\&T/SID Twelfth Color Imaging Conference: Color Science and Engineering: Systems, Technologies, Applications, Scottsdale, AZ, 9-12 Nov. 2004, pp. 65-69.

[22] C. E. Rodríguez-Pardo and G. Sharma, "Color control functions for multiprimary displays I: Robustness analysis and optimization formulations," IEEE Trans. Image Proc., vol. 29, no. 1, pp. 1152-1163, 2020.

[23] C. A. Athanasiadis, "Zonotopal subdivisions of cyclic zonotopes," Geometriae Dedicata, vol. 86, no. 1-3, pp. 37-57, 2001.

[24] V. I. Danilov, A. V. Karzanov, and G. A. Koshevoy, "Cubillages of cyclic zonotopes," Russian Math. Surveys, vol. 74, no. 6, pp. 1013-1074, 2019.

[25] F. Chavanon and E. Remila, "Rhombus tilings: decomposition and space structure," Discrete Comput. Geom., vol. 35, no. 2, pp. 329-358, 2006.

[26] G. D. Bailey, "Coherence and enumeration of tilings of 3-zonotopes," Discrete Comput. Geom., vol. 22, no. 1, pp. 119-147, 1999.

[27] G. H. Golub and C. F. V. Loan, Matrix Computations, 2nd ed. Baltimore, MD: The Johns Hopkins University Press, 1989. 